\title{
A PHENOMENOLOGICAL INQUIRY INTO THE NORTH AMERICAN SHAMAN'S EXPERIENCE OF THE ALTERED STATE OF CONSCIOUSNESS
}

\section{ROBERT ANDERSON}

\author{
Thesis submitted to the University of Saint Paul \\ in partial Fulfillment of the requirements for the \\ Doctor of Philosophy
}

\author{
School of Counselling, Psychotherapy and Counselling \\ Faculty of Human Sciences and Philosophy \\ Saint Paul University
}

(C) Robert Anderson, Ottawa, Canada, 2019

Keywords: experience of shaman, altered states of consciousness, perception 


\section{COMMITTEE MEMBERS}

Committee Chair: Dr. Judith Malette

Full Professor: School of Counseling, Psychotherapy and Spirituality Saint Paul University

Internal-Internal evaluator-committee Member: Dr. Martin Rovers

Full Professor: School of Counseling, Psychotherapy and Spirituality Saint Paul University

Internal-Internal evaluator-committee Member: Dr. Cynthia Bilodeau

Associate Professor: School of Counseling, Psychotherapy and Spirituality Saint Paul University

Internal-External evaluator-committee Member: Dr Lorraine Ste-Marie

Associate Professor: Providence School of Transformative Leadership and Spirituality, Saint Paul University

External-External evaluator-committee Member: Dr Nicole Yves Associate Professor: School of Social Work, McGill University 


\section{ACKNOWLEDGEMENTS}

I would like to thank my wife Cheryl for her continual support and love throughout the final two years of this process. She has taught me how to be a better person, and to never give up on my goals. Without her, I would not be here. To my family whose unending support was always there, I thank each and every one of you. To my friend and mentor Debbie McConkie, whose guidance and support over the past fifteen years has been without question, the reason why I have been able to succeed in my career. To my friend Helen, who's unwavering support, especially during the first two years, was essential to my inner development. I would also like to extend my sincere gratitude to my thesis advisor Dr. Judith Malette for her encouragement, understanding and steady approach. Her experience, insight and guidance has left a lasting impression on me. From the first day of the program up until this day Dr. Malette's support has never waned. It has been a true honour to work with her.

To my thesis committee, Dr. Cynthia Bilodeau and Dr. Martin Rovers who have both provided a depth of knowledge that has helped shape me as a researcher and writer. Dr. Bilodeau's help with my methodological approach was integral to the development of the thesis. Without Dr. Rovers, the essence of the Participant's answers would not have been reflected. I thank both of them deeply for their keen observations and helpful comments.

I would also like to thank Dr. David Joubert who has been a main contributor to my professional development as a psychotherapist. His understanding of psychological theory is without question the best I have come across. Finally, I would like to extend my gratitude and thanks to all of the participants in the study. My eyes have opened, and my understanding of consciousness has been expanded. Words simply do not come close to the graciousness I feel. 


\begin{abstract}
Although conflicting ideas exist about the origin of the shaman, it is clear that these individuals exist across culture and history (Eliade, 1964/2004; Winkelman, 2010). Research has indicated that a specific altered state of consciousness (ASC) exists that the shaman utilizes to help the individual or group heal (Harner, 1980). Discrepancies in understanding the shaman`s experience of ASC had led to the call for further research in this area. (Cardeña, 2011a; Walsh, 2007, Winkelman, 2010). This phenomenological study aimed to investigate the shaman`s experience of the ASC. Results indicated that the shaman's evolution was based on the criteria associated with the wounded healer archetype as well as conditions associated with the individuation process of Jung (1959/1980a). The study also revealed the shaman's experience of the ASC was accomplished through a pattern of development that resulted in each participant acquiring the status of shaman. Specifically the ASC was the avenue whereby each participant became a shaman. As well, the results indicated that the induction procedures associated with entering the ASC were not required as once the shaman achieved his/her status access to the ASC was readily available. Based on the narratives of the five participants, the results suggest that the shaman is a person who has an expanded understanding of the consciousness experience. This contrasts what Western society has understood about consciousness.
\end{abstract}




\section{TABLE OF CONTENTS}

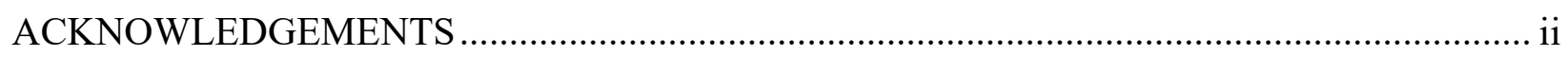

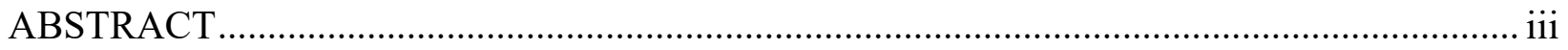

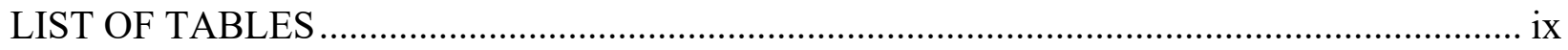

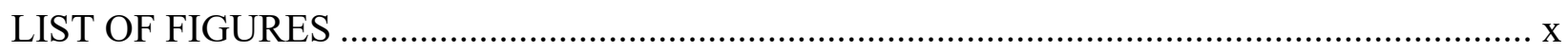

\section{CHAPTER}

I

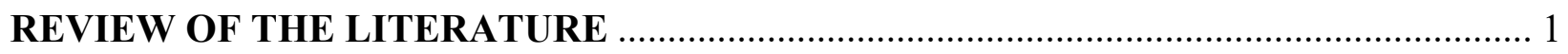

Statement of the Problem ....................................................................................... 3

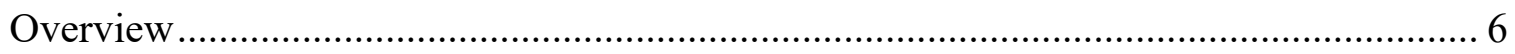

II

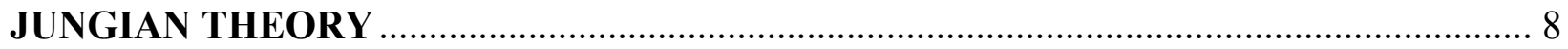

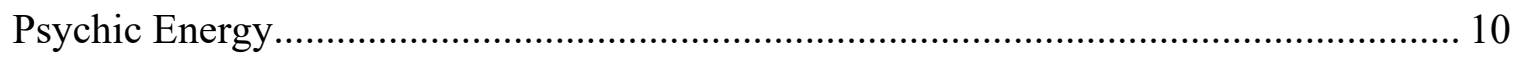

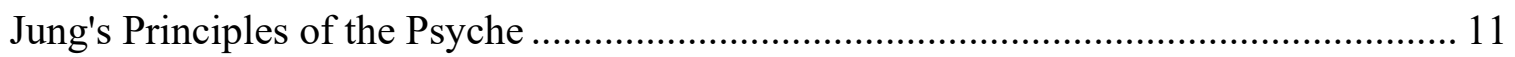

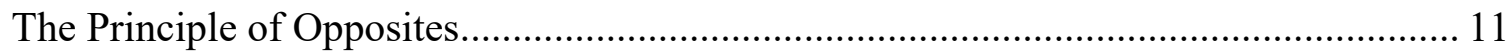

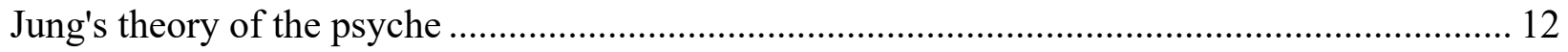

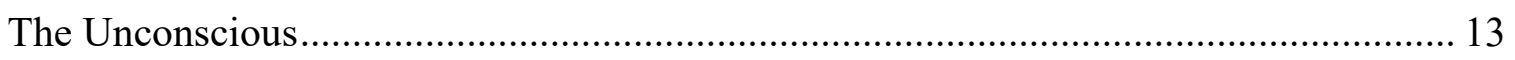

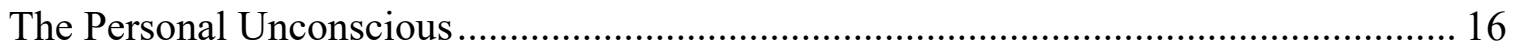

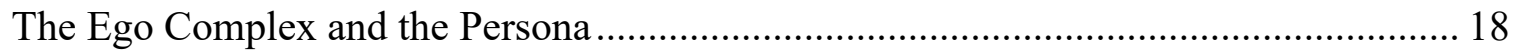

The Collective Unconscious ................................................................................... 20

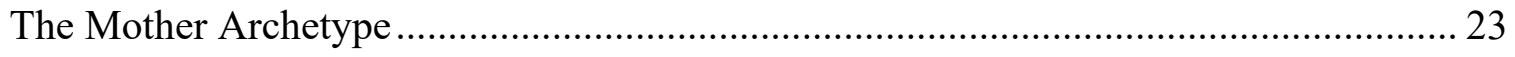

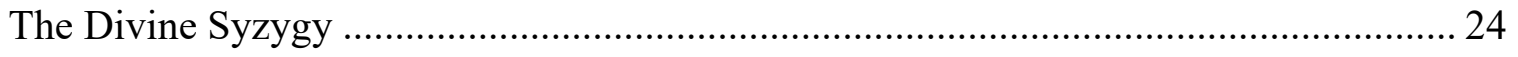

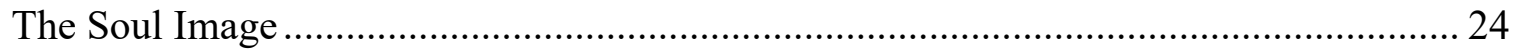

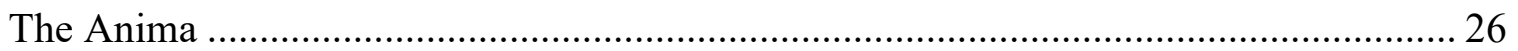




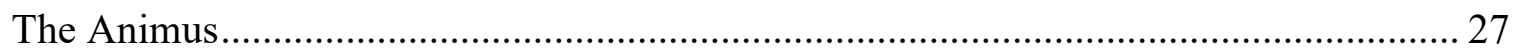

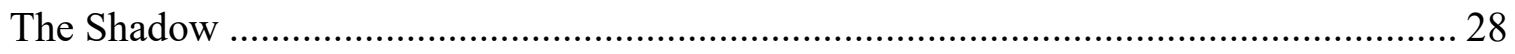

The Wounded Healer Archetype ………………………........................................... 29

The Self and the Individuation Process ........................................................................ 35

\section{III}

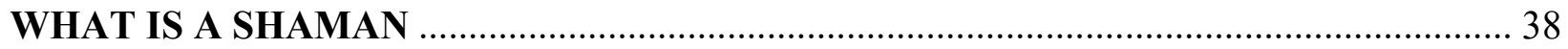

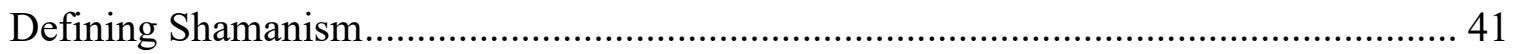

The Shaman as the Wounded Healer .............................................................................. 51

IV

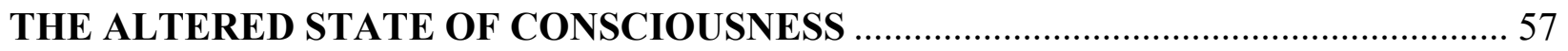

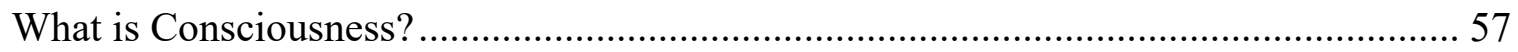

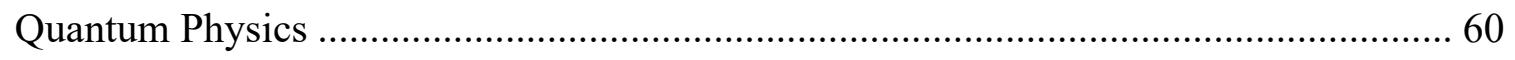

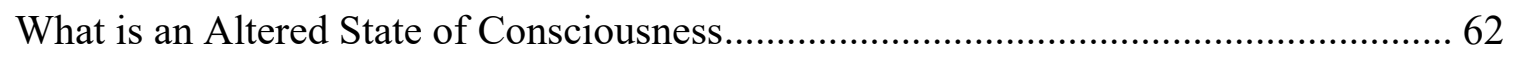

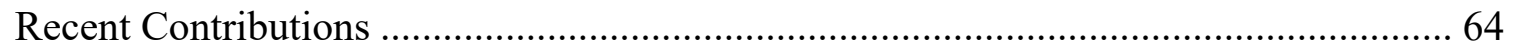

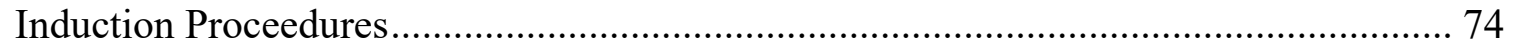

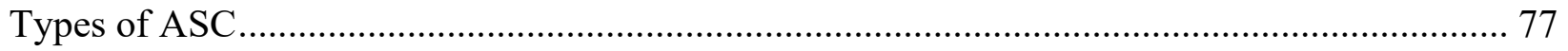

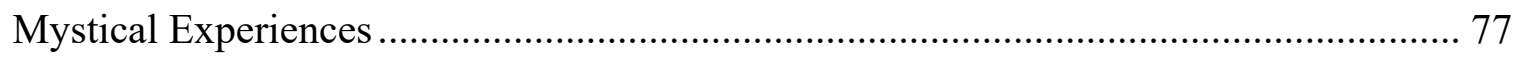

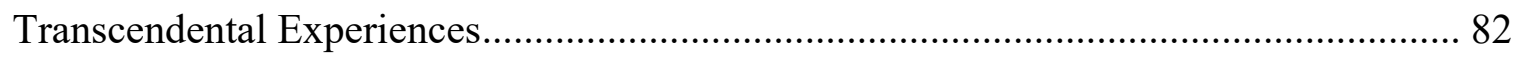

Religious/Spiritual/Mystical Experiences..................................................................... 84

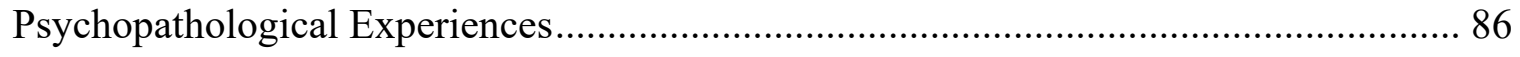

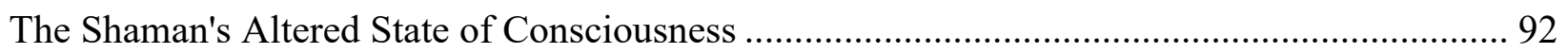

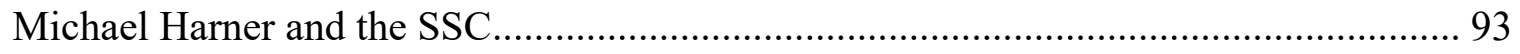

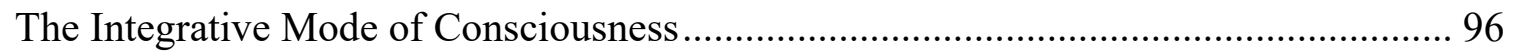

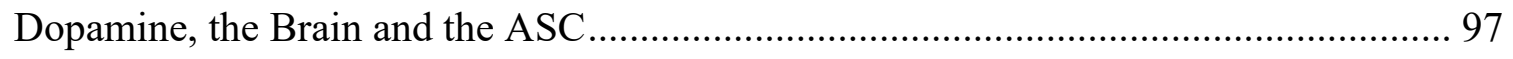




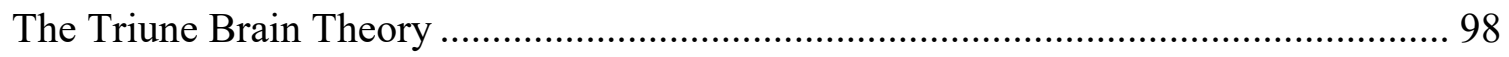

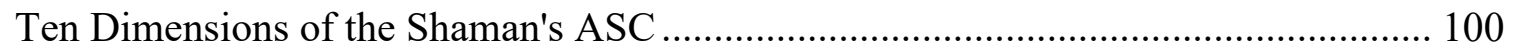

V

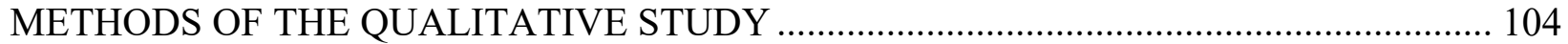

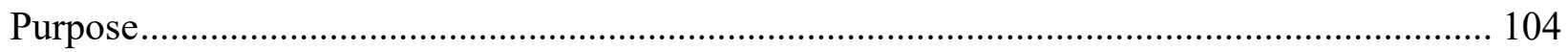

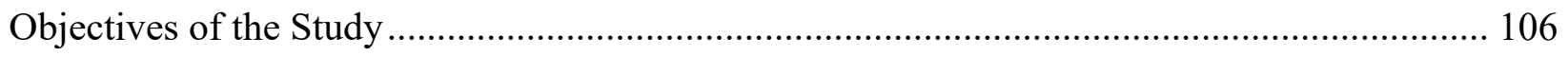

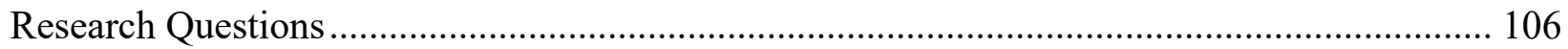

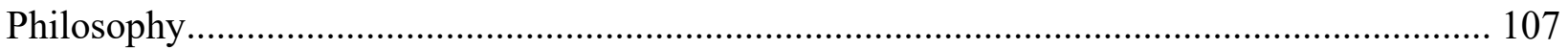

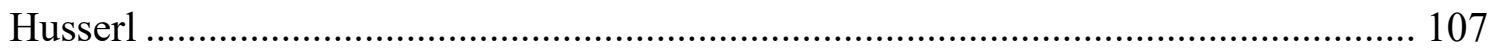

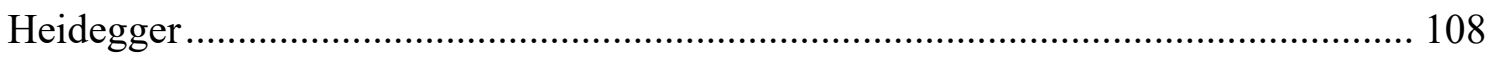

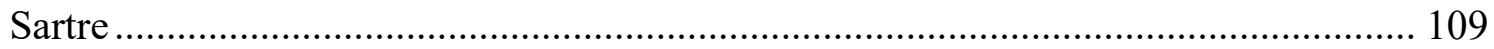

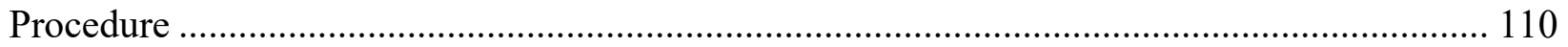

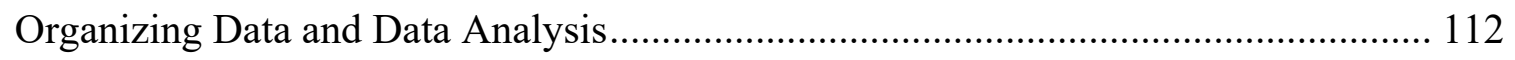

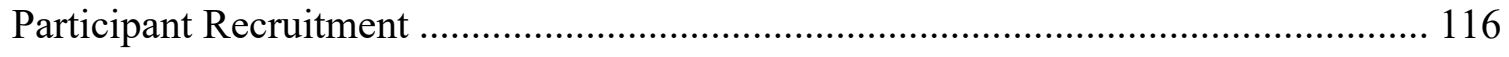

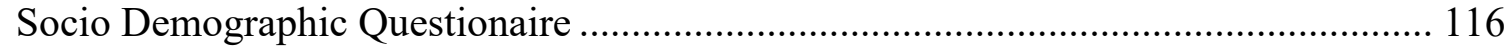

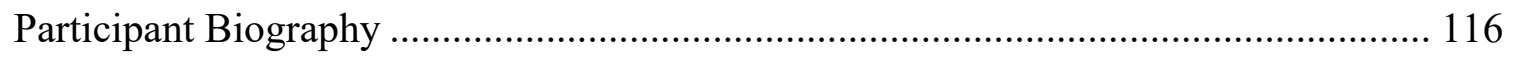

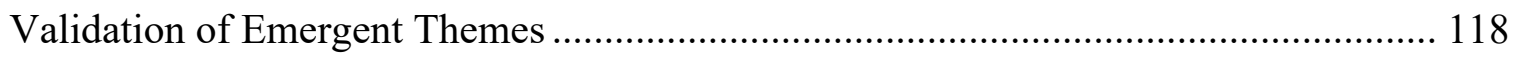

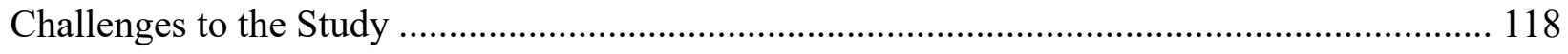

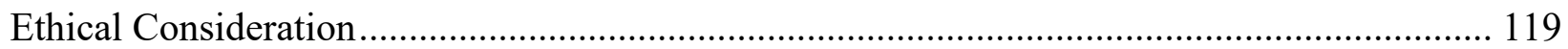

VI

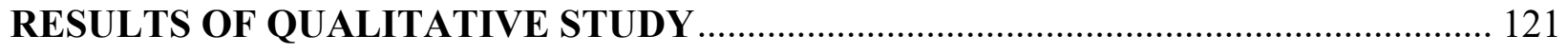

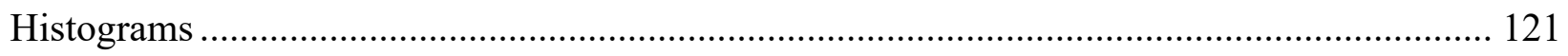

Emergent Theme: The term I used to identify myself as .................................................. 122

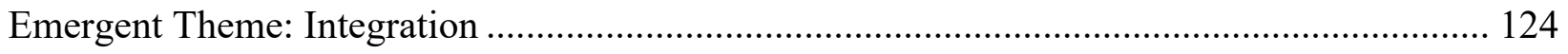


Emergent Theme: Learning a New Language .....

Emergent Theme: Teachers

Emergent Theme: Transmission

Emergent Theme: Application

Emergent Theme: Archetypal Examples of Individuation

VII

DISCUSSION

Reflections on the Research Questions..... 148

Emergent Theme: The term I used to identify myself as 152

Emergent Theme: Integration 155

Emergent Theme: Learning A New Language 157

Emergent Theme: Teachers 158

Similarties between The Collective Unconscious and the Meaning Unit: Teachers ..... 160

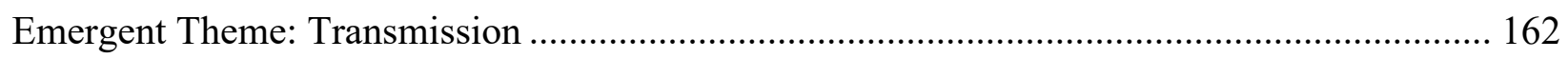

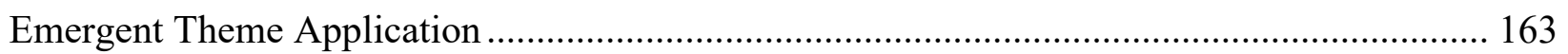

Emergent Theme: Archetypal Examples of Individuation ................................................ 164

Clinical Observations in the Death and Rebirth Process ....................................... 166

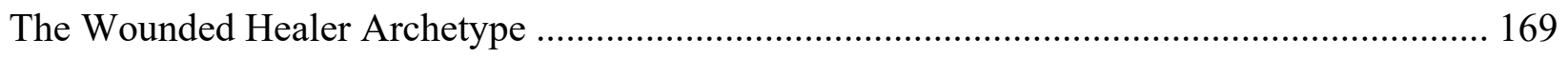

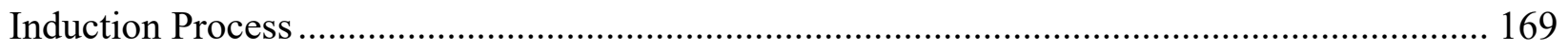

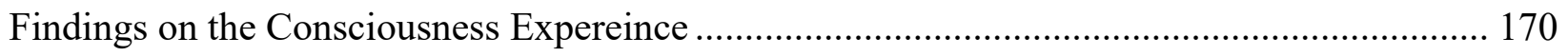

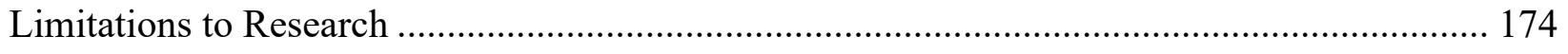

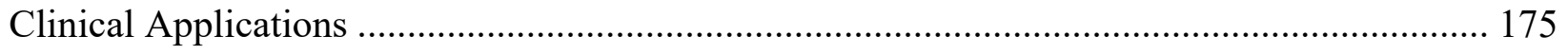

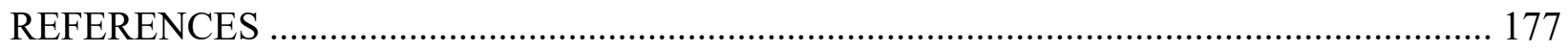

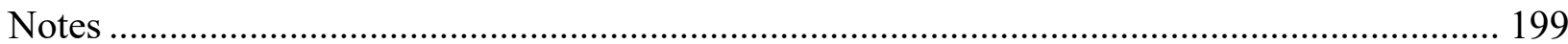


APPENDIX A: EMAIL VERIFICATION ................................................................... 201

APPENDIX B:RECRUITMENT EMAIL .................................................................... 206

APPENDIX C:CONSENT INFORMATION FOR PARTICIPANTS.................................... 207

APPENDIX D: SOCIO DEMOGRAPHIC QUESTUIONAIRE ......................................... 209

APPENDIX E: 3 STAGE SOCIO-DEMOGRAPHIC INTERVIEW .................................... 211

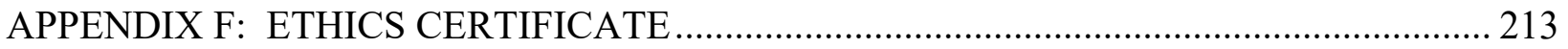




\section{LIST OF TABLES}

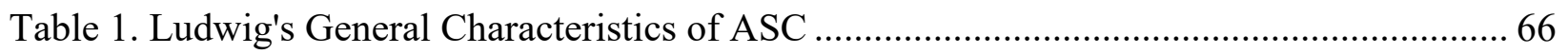

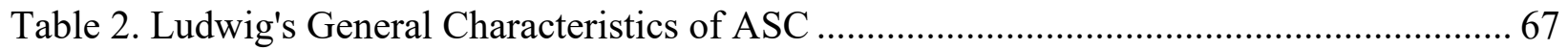

Table 3. Experimental Criteria for Detecting and Altered State of Consciousness.................... 70

Table 4. Experimental Criteria for Detecting and Altered State of Consciousness .................... 71 


\section{LIST OF FIGURES}

Figure 1. Jung's Model of the psyche with examples of Mother Archetype/Mother Complex .... 13

Figure 2. Healing between Psychotherapist and Client ................................................. 31

Figure 3. Fischer's: A Cartography of States of Consciousness ......................................... 73

Figure 4. Krippner's Model of Dissociative Experiences ............................................... 91

Figure 5. Template for Coding a Phenomenological Study............................................... 112

Figure 6. Protocol for assessing the reliability of the initial coding ...................................... 115

Figure 7. Emergent Theme: The term I used to identify myself as ..................................... 122

Figure 8. Emergent Theme: Integration ..................................................................... 124

Figure 9. Emergent Theme: Learning a New Language .................................................. 127

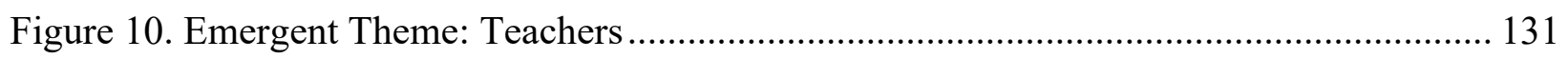

Figure 11. Emergent Theme:Transmission ............................................................... 135

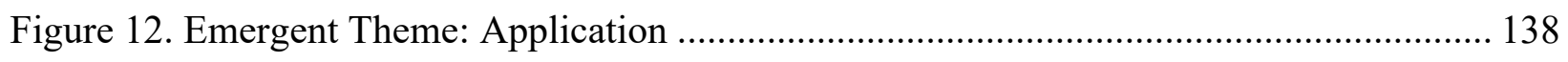

Figure 13. Emergent Theme: Archetypal Examples of Individuation ................................. 141

Figure 14. The Shaman's model of Development ........................................................ 150 


\section{CHAPTER I}

\section{REVIEW OF THE LITERATURE}

Creating alterations in physiological and psychological awareness (Ludwig, 1966), the Altered State of Consciousness (ASC) defies logic, alters time, changes perception, while creating a sense of joy and awe in the individual (Tart, 1975). Previc's research on the neurotransmitter dopamine revealed that the potential for the ASC exists across humanity (Previc, 2011). Expanded upon in Chapter IV in the ASC section, Previc's research focused on the neurotransmitter dopamine arguing that human behavior evolved via epigenetic factors that led to an elevation of dopamine in the human brain. This is significant because dopamine has been implicated in parasympathetic action on the body such as lowering blood pressure, reducing temperature and reducing oxygen consumption. Previc notes that parasympathetic action of dopamine is an important clue in accounting for the ASC experience (Previc, 2011).

Noted across culture and religion, the ASC has become the means to access levels of consciousness that are unlike what is experienced normally (Cardéña, 2011a). However, what is the normal conscious experience? Research in shamanism has indicated that an ordinary reality, and a non-ordinary reality exist simultaneously (Harner, 1980/1990). As well, Quantum Physics suggests that our conscious experience is structured by our observations of it (Wolf, 2001, 2004). Thus, is one experience different from the other, as Harner (1980/1990) suggests, or are both simply part of the overall conscious experience and determined by how we observe it (Wolf, 2001, 2004)?

Further to this, the shaman has been identified as an individual who has learned how to use the ASC mindfully to attain pertinent outcomes for the individual or group that is being helped (Eliade, 1964/2004, Harner, 1980/1990; Winkelman, 2010, 2011; Walsh, 2007). Neurological studies in mystical and transcendental states have offered research insight into 
where the ASC occurs in the brain (Girand et al., 2007; Cardeña, 2005; Beauregard, 2011a; Levin \& Steel, 2005; Newberg \& Lee, 2005). For example, Girand et al. (2007) note that the ventral pathways of the brain have been to play key roles in dreams, out of body experiences, floating sensations, deep hypnosis and hallucinations. This will be explained in more detail in Chapter IV. Similarly, Winkelman's (2010) study on shamanism suggests that a synchronization of the paleo-mammalian, neo-mammalian and reptilian brains occurs to create the ASC in the brain. As well, psychoactive drug interaction studies have furthered this, identifying how the ASC is created in the brain through neurotransmitter responses (Presti, 2011). Despite the neurological responses being similar in human brain interactions, research has indicated that the ASC is a subjective event that is experienced by the individual who is induced into an alternate reality (Fisher, 1986; Tart, 1975). This has caused considerable confusion in the validity of what is occurring subjectively as individual accounts vary (Cardeńa, 2011a; Tart, 2011, 1975). This has also become an issue in research concerning the shaman (Krippner, 2012).

It has been seen that each culture offers a unique expression to the conscious experience (Whitehead, 2011). When this becomes altered, one's perception of reality changes. Although accepted as an aspect of the conscious experience by Eastern belief systems such as Buddhism and Yoga, this has challenged Western research bringing it to ask fundamental questions that challenge our current belief systems addressing reality (Bohm, 1980). It is the act of separating that has limited our view of what physicist David Bohm refers to as a systematic division of wholeness that has been occurring for centuries. Occurring across language, culture, belief and theory, it has broken down our conceptual framework of reality into a system of fragmented parts (Bohm, 1980).

Although conflicting ideas exist about the origin of the shaman, these individuals exist across culture and history (Eliade, 1964/2004; Winkelman, 2010). Research has indicated that a 
specific ASC exists that the shaman utilizes to help the individual or group heal (Harner, 1980/1990). Discrepancies in understanding the shaman`s experience of ASC had led to the call for further research in this area. (Cardeña, 2011a; Walsh, 2007, Winkelman, 2010). Therefore, this phenomenological study aims to investigate the shaman`s experience of the ASC.

\section{Statement of the Problem}

While insight into the conscious experience has allowed research to grapple with fundamental questions about our existence, it has also alluded science as to why it occurs (Cardeña, 2011a; Chalmers, 1997). Thus, the overall picture of what reality is, remains difficult to explain. Concluding that it is in the definition of consciousness where science must turn, Revonsuo, Kalio \& Sikka, (2009) note that explaining the cognitive and behavioural functions of the conscious experience remains to be a challenge. Thus, if science cannot explain the conscious experience how can it explain the ASC? Despite this, Cardeña (2011a, 2011b) concludes that science has advanced the definition of the ASC. This has been seen in phenomenological studies such as in Ludwig's (1966) and Tart's (1975) work on classification (see chapter 4). As well, ethnographic studies on shamanism by Winkelman (2010), Walsh (2007) and Vaitl et al. (2005) helped clarify the ASC as a worldwide phenomenon.

Despite research indicating that the shaman is an individual who utilizes the ASC for the well-being of the individual or group being served, methodological issues continue to plague this phenomenon (Krippner, 2000; Walsh, 2007; Winkelman, 2010). These include Eliade's (1951/2004) conclusions that the shamanic ASC is limited to a deep form of ASC, and researcher's Winkelman (2010), Harner (1980/1990) and Hulkrantz (1985) who describe it consisting of a fluctuating range of altered states. As well, even though commonalities exist with the ASC as seen in mystical states (Winkelman, 2010), hypnotic states (Overton, 1998) and meditation research (Winkelman, 2010), the shaman's use of the ASC is unique due to its 
transformational effect on others (Harner, 1980/1990; Walsh, 2007). Thus, defining and understanding what the shaman experiences from a subjective perspective remains unclear, and understanding how the shaman uses the ASC to acquire information for the individual or group he/she is helping also remains unclear.

Research conducted on the societal implications of ASC (Whitehead, 2011) suggests that beliefs are determined by the culture people live in. For example, in his research on shamanism and clinical hypnosis, Overton (1998) claims that neurophysiological evidence indicates that the shamanic ASC differs only in cultural context. This is critical due to the cultural indicators that enable the shaman to utilize the ASC for the benefit and well-being of the people. Therefore, if truth is generated by the cultural beliefs of a society, then what is truth? Bohm (1980) notes that when we use our theories to observe the world, the factual knowledge we gather is inevitably shaped by them. This is important to consider as evidence on the ASC suggests that we may be "altered" from what we really are (Whitehead, 2011). Furthermore, research has identified that the shaman observes reality as having two distinct modes of consciousness, the normal state and the non-ordinary state (Harner, 1980/1990). Thus, if the shaman can enter non-ordinary reality then what is the shaman entering? And, what allows the shaman to achieve this?

Answers to these questions may lie in research on induction procedures of the ASC. For example, comparisons on induction procedures of the West to that of the shaman include the use of rhythmic drumming to induce the ASC (Beischel, Rock \& Krippner, 2011), psychoactive drug use, such as peyote (Schaefer, 2011), lucid dream states (Winkelman, 2011) and hypnotic like procedures (Cardeña, 2011a). The use of rhythmic drumming has been seen to induce a theta brain state as seen in meditation studies (Winkelman, 2011). For example, through the process of extreme dancing the shaman achieves this by exhausting himself and collapsing into an ASC where he remains conscious (Winkelman, 2011). As well, studies focusing on psychoactive drug 
use identified that the areas of the brain responsible for allowing sensory, visual and auditory changes are altered in individuals who have ingested them (Schaefer, 2011). Moreover, understanding how the shaman uses the induction procedure to achieve transformation in others will offer clarity into this phenomenon.

From his ethnographic research, Winkelman (2010) reflects that shamanism has evolved using traditions for creating, manipulating, and using an assortment of personas for psychological and social integration. Suggesting that shamanism finds its roots in meditative traditions allowing for the control of attentional, perceptual and conceptual aspects of consciousness, Winkelman (2010) mentions that the shaman uses mythological systems to interpret human nature. The researcher suggests that a belief in spirit reflects a fundamental human need to interpret meaningful occurrences throughout life and is based on the metaphoric extension of the self to the divine. However, in his research, Walsh (2007) concludes that spirits are experienced as an interaction that is separate from the ego and is felt to be intelligent and nonmaterial. As well, Harner (1980/1990) suggests that the spirit world is not a metaphoric representation, rather a legitimate experience giving the shaman critical information required to help the individual or individual in need. In his work, Eliade (1951/2004) concludes that a definitive quality of the shaman is the ability of inducing trance states enabling the shaman's soul to leave his body. Also known as the shamanic journey, the shaman does this to access information from the spirit world (Walsh, 2007).

For the shaman, this interaction is critical as it can provide information required to help the individual(s) in need (Walsh, 2007). Thus, even though the ASC is accessed through various induction procedures, the shamanic ASC is unique due to the following characteristics: (1) soul flight (Walsh, 2007), (2) cultural symbolic representation (Winkelman, 2010; Walsh 2007) and, (3) its intention to offer healing and change in the individual or community. Consequently, a 
distinguishing feature of the shamanic ASC is its capacity to act as both a neurophysiological and cultural/psychosocial occurrence (Craffert, 2011) utilizing specific culturally defined symbols to create changes in the individual or community (Coady \& Hill, 2003; Mazzola, 1998). Therefore, the ASC of the shaman will be generally defined as: a set of experiences whereby the shaman enters an induced altered state with a degree of control that enables the shaman to access information that is used for the benefit of the individual or community being served (Walsh, 2007; Winkelman, 2010). Considering that research has indicated that the ASC is not a controlled experience, the questions that elude research include: (1) how does the shaman remain conscious during the altered state using a degree of control to obtain information for the wellbeing of others; And (2) what makes the shaman different from others who have experienced the ASC? The shaman's ASC experience has been noted to be unique and categorically different from what has been reported on the ASC in the literature (Winkelman, 2012). Therefore, this study will be investigating this notion while considering the research that has been done on the subject of ASC. This study will investigate the shaman's experience of the ASC through participant interviews to add clarity and understanding to the nature of the experience.

\section{Overview}

This thesis is divided into seven chapters. The first chapter presents an introduction to the study, the main issues concerning the ASC, the shaman's ASC and the limitations in the research thus far. Chapter two focuses on Carl Jung's theory of the psyche and the collective unconscious, the archetypes, the wounded healer archetype and the individuation process that has been associated with the shaman. Chapter three presents the reader to the shaman, offers historical relevance, relevant theory, and the shaman as a wounded healer. Chapter four considers the ASC with an overview of the research on consciousness and the ASC, while also discussing the 
shaman's ASC. Chapter five offers the methods of the study, chapter 6 the results, and chapter 7 the discussion.

As a starting point, the current study will use Walsh's (2007) phenomenological description of the ten dimensions required for defining the shaman's experience of the altered state. Walsh (2007) has offered a comprehensive outline of the steps required for the shaman to navigate the ASC. These include: (1) degree of control (used mindfully to achieve relevant results for the individual or group that is being helped), (2) awareness of the environment, (3) ability to communicate, (4) concentration, (5) degree of energy or arousal, (6) degree of calm, (7) emotion, (8) sense of identity, (9) out of body experience (OBE), and (10) the nature of inner experience. Walsh (2007) notes that the shaman's life and training reflect an archetypal process of the psyche, allowing for "considerable healing potential" (p. 74) to be established, and the above criteria to emerge. Reflecting on the death and rebirth process, whereby the shaman undergoes a transformation in the psyche, Walsh identifies strong similarities to what Carl Jung refers to as the wounded healer archetype (Walsh, 2007). What follows will be an account of Jung's theory and wounded healer archetypal description. This will allow for a psychological view point of the shaman and reflection into how Jung understood the psyche. 


\section{CHAPTER II}

\section{JUNGIAN THEORY}

Carl Gustav Jung (1875-1961) was much more than a psychiatrist, he was an important thinker of his time. Jung's views of psychology took on new meaning with his insight extending into the disciplines of physics, philosophy and spirituality (Jung, 1963/1990; Knox, 2003). This approach separated Jung from his contemporaries and set him upon a new course of understanding as he recognized the limits of his profession (Stevens, 1994). Although it is true that his break from Freud acted as a catalyst for Jung's views to fully manifest, the foundation of his thought began while he was still a boy (Stevens, 1994). This early stage of development is important to understand as it gives context to what Jung offered the world.

Insights into his own childhood are reflected in Jung's autobiography, Memories, Dreams, Reflections (Jung, 1961/1990). He recalls that his mother was the youngest daughter of a clergyman who was both popular and eccentric. His grandfather's interest in the occult, medicine, theology, and spiritualism all were to have considerable influence on Jung (Stevens, 1994). Jung recalls his memories of his home being unpleasant, describing it as "unbreathable" (Jung, 1961/1990, p. 33). These created feelings of dread, melancholy within Jung as the visible tension between his parents grew (Jung, 1963/1990). At the age of 3yrs old, Jung's mother entered a hospital after having a breakdown, one that Jung never fully recovered from (Stevens, 1994). The combined experience of his mother's illness and the tension between his parents, added to the already growing sense of detachment he had from the outer world. (Stevens, 1994).

During this time, Jung recalls feeling mistrustful about love and a feeling of unreliability towards women (Jung, 1961/1990). This contributed to his introverted nature, a term he later expanded upon in his theory of personality types (Stevens, 1994). During his childhood, Jung's 
withdrawn and aloof approach to himself and the world, made him unpopular, causing him to spend a considerable amount of time alone. In his reflections, Jung notes, "I liked that best. I played alone, daydreamed or strolled in the woods alone, and had a secret world of my own" (Jung, 1961/1990, pp. 67-68).

Jung noted that his relationship with his father was difficult for him. His father maintained the outer image of kindness and tolerance. Even though Jung saw his father as reliable, he also saw him as powerless and emotionally unstable (Jung, 1961/1990). Jung recalls that early in his father's ministry, he lost his faith, however felt obligated to continue his parish duties, having no other recourse (Stevens, 1994). This impacted Jung as his own experiences with God contributed to the struggle he had with his father. During a series of events that occurred, Jung felt that he had a personal experience of God's grace that shaped his understanding of what it means to be a human being. From this, he concluded that surrendering to God's will is a vital step in selfrealization and growth. Jung realized that God's will stood above all human rules, apart from religion and the church. In an attempt to repair the rift between them, Jung would engage in conversations with his father in his early adulthood hoping to help his father grow out of his narrow stance, however concluded that it was beyond repair (Jung, 1961/1990).

Examples of his early insights often came to him when he was out on his own. Jung recalls that while alone with himself, he would stand on large stones and reflect: "I am sitting on top of this stone and it is underneath" and he imagined the stone would reply to him: "I am lying here on this slope and he is sitting on top of me." Jung would then ask of himself: "Am I the one who is sitting on the stone or am I the stone on which he is sitting" (Jung, 1963/1990, p. 34)? Stevens (1994) notes that questions like these posed by Jung showed an early understanding of projection. As Jung matured, he became distinctly aware of being two persons. This he intoned, 
was not a conscious experience during his childhood, rather one that he "sensed with an overpowering premonition and intensity of feeling" (p. 64). Later he began referring to them as No. 1 and No. 2. No. 1 was the schoolboy impacted by his experiences of life, while No. 2 lived where he was completely aware of the cosmos, where also the human mind and God were connected. This altered state of awareness was the collective unconscious and allowed for Jung to experience his wholeness (Knox, 2003).

These inclinations show us that Jung's insight and focus began early, setting the stage for a lifetime of study and postulations. As Jung's understanding evolved, he regarded that the psychology of his time was as an explanatory science. This eventually led to a correspondence with Sigmund Freud, one of the most regarded relationships that had major contributions to the foundations of psychological thought. Firstly, as his student, and later his colleague, Jung's time with Freud began as a father-son relationship (Jung, 1963/1990). This slowly began to dissolve as Jung grew past Freud's views, eventually establishing analytic psychology. Jung concluded that Freud's psychology utilized an etiological focus that was mainly personal in nature (Stevens, 1994). The main catalyst contributing to their break up was Jung's position on the existence of the collective unconscious and disagreement of two of Freud's basic premises. These were: (1) that human motivation is sexually based and (2) that the unconscious mind is personal and peculiar to each person (Stevens, 1994).

\section{Psychic Energy}

Jung came to understand that a naturally occurring energy exists in each individual. He called this libidinal energy. This was also quite different from Freud's view, who thought libido was the energy of the psyche and primarily based on sexual energy. Seeing human behavior motivated by drives or instincts, Freud thought behavior to be a neurological manifestation of 
our physical needs. Considering sex as a primary social need, he considered it an important aspect to the dynamics of the psyche, thus seeing libidinal energy as its main expression (Storr, 1989). However, for Jung, libido is a generalized life force with sexuality being one expression of it (Stevens, 1994).

Looking to physics, Jung used Robert Mayer's theory of transformation of energy to help him arrive at a generalized concept of libidinal energy. In so doing, he concluded that all physical phenomena are manifestations of energy (Jung, 2014). Noting that energy is only experienced as a motion and force, it appears in two ways through: (1) action, or (2) potential. Psychic energy appears through actual means in the specific phenomena of the psyche, such as by instinct, wish, will, affect, attention etc. Jung refers to these as the psychic forces. When it occurs through potential, the psychic energy reveals itself by specific achievements, possibilities, aptitudes, attitudes etc. Jung refers to these as the psychic states (Jung, 2014).

\section{Jung's Principles of the Psyche}

Throughout his work, Jung's understanding of psychology remained steadfast to the personal and transpersonal developmental nature of the psyche and offered a constructive and expanded criticism to its development (Jung, 1959a). Jung would note that the personal and transpersonal are intertwined and cannot exist alone (Jung, 1963/1990). The interwoven nature of the psyche involves specific principles that Jung would refer to throughout his theory of psychology, especially in the archetypes. Although a complete understanding of the principles Jung used is worthwhile, for the purposes of this thesis, the principle of opposites will be discussed.

\section{The Principle of Opposites}

From the Greek philosopher Heraclitus, Jung discovered the process of enantiodromia. The 
process took shape in life when an overabundance of a phenomenon occurred, leading to the experience of its opposite (Stevens, 1994). Referring to this as the principle of opposites, it operates in our daily lives occurring in our thoughts, our actions, and in our beliefs. Jung used the term enantiodromia to describe the emergence of the unconscious opposite to occur over time. Jung mentions it almost always occurs when an extreme, "one-sided tendency dominates conscious life" (Jung, 1971/1991, p. 393). Over time this counter position is built up, firstly inhibiting conscious performance and later breaking the conscious control of the individual (Jung, 1971/1991). Described later in the section concerning the collective unconscious and the archetypes, this is demonstrated in his theories of the archetypes as each archetype produces an opposite.

\section{Jung's theory of the psyche}

What will follow will be a description of the contents of the psyche as displayed in Figure 1. Jung's theory divides the psyche into three parts: (1) ego, (2) personal unconscious, and the (3) collective unconscious (Stevens, 1994). The contents of the psyche will be described in the following order: (1) The unconscious; (2) the personal unconscious, complexes, the ego complex and the persona; (3) collective unconscious and the archetypes: the anima, the animus, the mother, the shadow, the wounded healer, and the Self. Please note that in Figure 1 is an example of one of Jung's complexes, the Mother Complex, one that he lived with throughout his life (Jung, 1963/1990). This will also be explained below in the section concerning the personal unconscious where the experience of a complex occurs. Finally, the Individuation Process will be presented last to bring Jung's theory of the psyche together. 
$\mathrm{C}=$ Complex; $\mathrm{A}=$ Archetype; $\mathrm{MA}=$ Mother Archetype; $\mathrm{MC}=$ Mother Complex

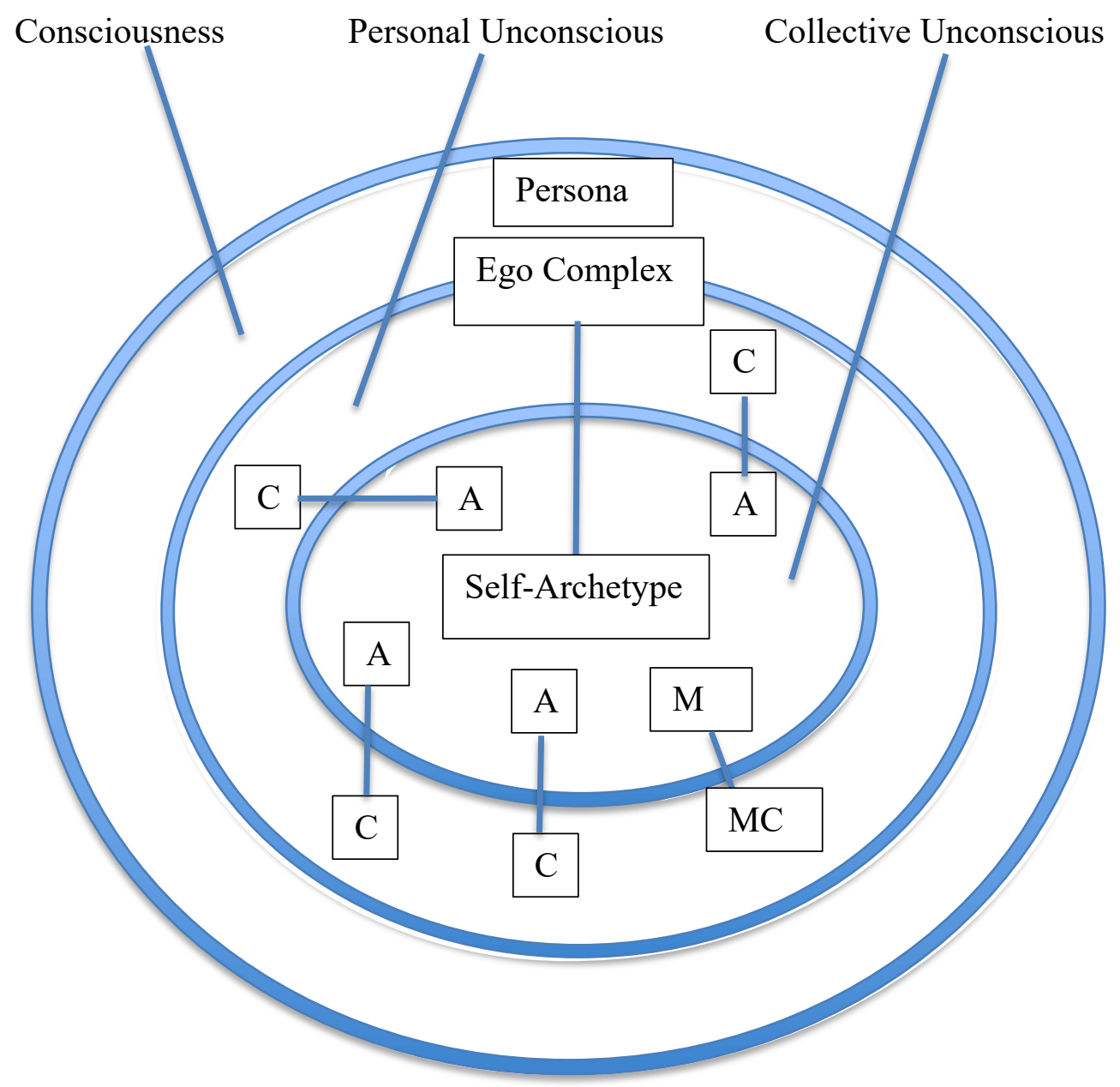

Figure 1. Jung's model of the psyche with the example of the Mother Archetype and Mother Complex. Jung (1961/1990) discussed his own struggle with his mother and the archetypal process he moved through. Figure 1 shows Jung's insight into his own psyche. Permission has been granted by Oxford University. Please see Appendix A for details.

\section{The Unconscious}

The unconscious consists of undifferentiated contents representing commonalities of human character. These result in the shared experiences of fear, danger, struggle, relational dynamics between the genders, and between children and parents. Its primary role is to interpret 
individual experience to help the psyche adjust and compensate for outer reality (Jacobi, 1942/1999).

Jung suggests that the unconscious has two layers, the personal and the collective. Jung agreed with Freud that the first layer is superficial and personal in nature, containing both temporarily forgotten and repressed memories (Jung, 1959b/1980). Unlike Freud who disagreed with the existence of the collective unconscious, Jung argued that it contained pre-existing forms, the archetypes, that contributed to the instinctual responses that we have to daily life (Stevens, 1994). Jung argued that not accepting these as a priori instincts common to humanity, was an oversight, as both have a significant influence on personal psychology (Jung, 1959a/1980).

Placing much of his attention into understanding the collective unconscious, he saw similarities between dream content and the mythology of humanity. Furthermore, Jung notes that myth allows one to connect personally to the individual's subjective experience whereas science is more objective and cannot be applied to the unique aspects of the individual's life (Jung, 1961/1990). Therefore, his position on the archetypes as inherited instincts, has remained controversial. This led to Jung's argument on the psyche of a newborn child contrasting with the tabula rasa or blank slate approach common to behaviorists (Knox, 2003). As Jung states:

It is in my view a great mistake to suppose that the psyche of a newborn child is a tabula rasa in the sense that there is absolutely nothing in it. In so far as the child is born with a differentiated brain that is predetermined by heredity and therefore individualized, it meets sensory stimuli coming from the outside, not with any aptitudes but with specific ones...These aptitudes can be shown as being inherited instincts and preformed patterns, the latter being the a priori and formal conditions of apperception that are based on instinct (1959a/1980, p. 1440). 
Revolutionary for its day, the existence of the collective unconscious has been largely overlooked by cognitive and evolutionary psychology (Knox, 2003). Due to the advent of behaviorism and subsequently the focus of cognitive psychology, understanding Jung's theory of archetypes as biological in nature, argues for phenomenological understanding of myth to be considered as evolutionary fact (Knox, 2003). This remains a sensitive topic in academic psychology, although few exceptions exist such as cognitive scientist Paul Gilbert who explored the link between Jung's concept of the archetype and emerging ideas in evolutionary psychology concerning innate mental structures. Gilbert emphasized that archetypes are important due to them being: "The evolved psychological mechanisms of the mind which guide our behavior in certain ways have evolved (like the defense system) because they evolved certain adaptive problems." (Gilbert, 1995, p. 142)

In her book, Archetype, Attachment, Analysis, Knox (2003) points out that a majority of studies on Jungian theory have examined Jung from a particular stance, due to the fact that no research could match the vast knowledge that Jung approached his theories from. This creates a bias, and therefore does not give full credit to his work. Thus, to understand Jung one must consider the reasons why he went across disciplines to further his work (Knox, 2003). This also suggests a reason for Jung's continual evolving understanding of the archetype. As well, it would explain why his theories were continually brought back to focus as he published. Jolande Jacobi's (1942/1999) book, Complex/Archetype/Symbol is one exception that provided academic psychology with an influential map on Jung's insights, providing support for archetypes, concluding that they can be approached from many angles. This is key to understanding Jung as he innately understood that the archetypal position and the a priori nature can only be understood from the vast amount of literature and critical thinking offered by academia. 


\section{The Personal Unconscious}

The experiences of the personal unconscious are accumulated during the individual's life (Jung, 1959a/1980). Being primarily made up of feeling-toned complexes, Jung's concept of the complex was initially presented during his work with Freud, who was intrigued by the idea, but later rejected it when they departed ways (Knox, 2003). Being influenced by the French psychologist Pierre Janet, it is speculated that Jung changed Janet's original idea of simultaneous psychological existences into the complex (Knox, 2003).

Jung maintained that a complex occurs due to the refusal to experience the suffering of a situation, rendering it to the unconscious. A complex is comprised of perception, feeling, volition and intention, as if an autonomous subject was present (Knox, 2003). Complexes are also comprised of a nuclear element consisting of two factors. Firstly, the complex is causally related to the environment and secondly determined by an innate aspect of the individual's character (Jung, 1954; 1959a/1980). The origin of a complex is usually traumatic, constituting memories, images, emotions and sensations that can create considerable unease in the individual (Kalsched, 1996;Knox, 2003).

The complex retains the image of a specific psychic situation latent with an abundance of emotionally charged group of ideas or images (Jung, CW 8, para 201). The complex clusters around a theme involving three components: (1) sense perception, (2) intellectual components, and (3) feeling-tone (Jung, CW 3, para 201). Jung notes that the feeling-tone is an "affective state" supported by somatic experience, hence the "feeling-tone" experience. The resulting emotion is then split off from conscious awareness, gradually fragmenting our understanding of ourselves into parts. The splitting results from the inability to experience the entirety of one's own personal nature. Eventually, this creates the development of dissociations and the creation of a feeling-toned complex. Although splitting is considered a normal aspect of life, it becomes 
pathological if the splitting of a complex becomes too strong and the emotional conflict unbearable (Sander and Beebe, 1984).

Complexes are strongly influenced by the archetypes, tending to have archetypal elements. In healthy individuals, complexes pose little issue, and can help to balance the one- sided position that the ego maintains. Once the ego is aware of the issue, it can adapt and assimilate the understanding of the situation. Complexes become a problem, however, when the ego is compromised and unable to determine what is occurring (Jacobi, 1942/1999). For example, if the ego's reflective ability is compromised, the complex has the capacity to function automatically possessing a compulsive quality (Knox, 2003).

Jung states that the power of the complex's autonomy includes an ability to appear dormant in the individual until brought to life by life events that seem to trigger it into action and manifest itself independently of the will. It can act in direct opposition to conscious tendencies by forcing itself tyrannically upon the conscious mind. This explains the increase of affect on the individual, that feels like the individual has been invaded. Also known as a possession, the complex's power is determinant upon the stability of the ego. Becoming terrifying and mysterious to the ego, the ego gives way to its power. Although its effect is dramatic, its intention is to protect the self-care system, and is thought to originate from early emotional experiences (Kalsched, 1996).

A complex is therefore inconsistent with who the person is. Having its own wholeness and autonomy, it can take control of the individual's consciousness and become the actors in our dreams that we are powerless against. Once this material is repressed the complex acts as a traveler passing between conscious and unconscious territory (Kalsched, 1996). Often confusing and difficult to put into words it is not possible to suppress completely (Jung, 1954). During his time at the Burghöl hospital, Jung developed a word association test with his colleagues. During these tests, subjects were asked to respond with the first word that came to mind while given a 
hundred words to answer too. When the responses took a long time to come, or the associated word was uncommon, nonsensical, forgotten, or accompanied by certain emotions, Jung thought this to be the indication of a complex, or a sign of an unconscious psychological conflict (West, 2013).

\section{The Ego Complex and the Persona}

Originating out of the Self archetype, Jung understood the ego to be a separate complex that "appears to possess a high degree of continuity and identity" (Jung, 1971/1991). Occurring on the subjective level, the nature of the personality is formed only partly from the ego (Jung, 1959b/1980). Comprised of thoughts, memories and emotions, the ego symbolizes the conscious aspect of the individual (Jung, 1916). It can be considered as the headquarters of the psyche, maintaining its position at the center of consciousness (Jung, 1971/1991). In Figure 1 on p. 13, the ego has been placed as existing in between the unconscious and consciousness. Jacobi (1942/1999) mentions that the boundary shifts depending on the individual.

Noting that it is both a content of and a condition of consciousness, Jung mentions that psychic elements become conscious due to the ego's awareness of it. When considering consciousness, Jung defined it as the "the function or activity which maintains the relation of psychic content with the ego (Jung, 1963/1990, p. 536). For the ego, its main concern is selecting how to interpret the most relevant information from the outer environment and the best course of action to take with it. The rest that the ego does not acknowledge is assimilated into the unconscious (Jung, 1971/1991).

Resting on two distinct criteria, Jung (1959b/1980) identifies the ego with somatic and psychic criteria. The somatic base of the ego is both conscious and unconscious. The conscious aspect is aware due to the somatic perceptions it has of its environment, making them psychic in nature. In other words, what one feels in the body allows for the somatic experience to become 
conscious. The experience of seeing a loved one after a long time away to the disappointment of not getting the job we wanted are but a few examples.

Through his own self observations and that of his patients, Jung noticed that the psychic content of the ego is comprised of three groups: first, the temporarily subliminal content that is duplicated voluntarily; second, unconscious contents that cannot be duplicated voluntarily; thirdly contents that are incapable of becoming conscious at all. Jung (1959b/1980) stresses that the ego is subordinate to the self-archetype and appears, at first, to have free will. By free will Jung means being able to make free choice allowing for the subjective feeling of freedom to occur (Jung, 1959b/1980).

The influence that the unconscious has over the ego is predicated upon its own understanding of the information it is providing. As Figure 1 shows, the self is directly influencing the ego through unconscious material, impacting free will. Being unconsciously influenced by the self, the ego is therefore limited and free will is predicated upon the ego's interpretation of what is occurring. Thus, it can be involuntarily assimilated by unconscious aspects of the personality, being greatly affected by them (Jung, 1959b/1980). An expanded description of the self will be offered in the section on the archetypes.

Situated above the ego complex, is the persona. The persona is the aspect of the personality existing for the purposes of adaptation to the external world. Thus, all professions or callings, has its own characteristic persona (Jung, 1959a/1980). According to Jacobi, the persona acts as a compromise "between the demands of the environment and the necessities of the individual's inner constitution" (Jacobi, 1942/1999, p, 385). The persona acts as a barrier to ensure the individual can interact with the environment in a relatively well-ordered fashion. It can become an issue due to the conciliatory nature it offers. Thus, the persona can become a "grown on mask" (Jacobi, 1942/1999, p. 390) causing the individual to shrink to the demands of the world. 


\section{The Collective Unconscious}

Jung maintained that the collective unconscious exists in all individuals. Containing preexisting forms known as the archetypes, they operate within our unconscious guiding our life through a variety of experiences (Jung, 1965a/1980). Often accomplished through projection, Jung maintained that this causes confusion and misunderstanding as the person is not aware of the archetypal process occurring. Thus, a projection is an unconscious motivation of one's own archetypal issue, being transferred onto another person or situation. The result is a mistake in understanding whereby the person assumes something is being done to them, whereas it is being offered to them by their unconscious to help them understand what is occurring (Jacobi, 1942/1999).

Noting that the archetype originates from the Platonic, Jung saw the archetypes as having the ability to induce, control, and facilitate the behavioral characteristics and common experiences of human beings (Jung, 1959a/1980; Stevens, 1994). Kalsched (1996) mentions that all archetypes have a bipolar dynamic with one aspect representing instinctual reactions that occur in the body, and the second represented by a spiritual component consisting of images produced by the mind.

Two types of archetypes emerge in our individual experience: one as active personalities in dreams and fantasies, and the other, during our daily lives (Jung, 1959a/1980). When actualized or experienced, the archetype manifests into our lives as definitive forms to the specific aspects of our individual psychic contents (Jung, 1959a/1980). This means that they do not manifest literally however create a subjective experience in our life that is interpreted through the archetype that is being experienced. Also known as the constellation process, it allows for the potential of it to be realized by the individual, rendering it capable of conscious reality. (Jung, 1959a/1980). When activated, it is an unforeseen event revealing itself through the 
experiences that are encountered throughout life.

As the descriptions of the archetype are amassed in the exhaustive literary work compiled by humanity, Jung also saw commonalities existing in primitive culture (Jung, 1959a/1980). Specifically, Jung emphasized anthropologist Lévy-Bruhl's concept of représentations collectives as being similar to the archetype (Jung, 1959b/1980). Jung supported Lévy-Bruhl's observations that the mentality of primitive societies originated from the mystical with the archetypes representing a pre-logical description in nature and following what Lévy-Bruhl called the law of participation (Mousalimas, 1990). For example, Lévy Bruhl notes that primitive people see themselves as equal to their surroundings as opposed to distinct from them (Bruhl, 1926/1966). As Mousalimas (1990) states, the law of participation allowed for an experience to be both singular and plural, itself and something else, being present here and also elsewhere. For example, at one glance the sky looks the same, and with another an image appears distinctly resembling an animal form potentially offering insight into the nature of an issue for the individual gazing at it. Thus, acting like seeds, these images have been planted into our psyche, inherited as a comprehensive system of thought that offers an understanding to the unconscious motivations of humanity (Neuman, 1954/1999; Knox, 2003).

In his book, The Origins and History of Consciousness, Neuman (1954/1999) provides a detailed account of myth that describes the archetypal process as an evolution of consciousness from infancy into adulthood. Also arguing for the archetypes being the "pictorial form of the instincts" (p. 234), Neuman (1954/1999) maintains that the unconscious reveals itself through images that the conscious mind assimilates into life events. Furthermore, the archetype being an abstract concept is refutable as it can only be described in terms of its unique phenomenology (Jung, 1959a/1980). Neuman (1954/1999) explains this further suggesting that the archetypes from a structure in the psyche acting like organs that the individual depends upon, and 
conversely whose injury can be detrimental. This explains why Jung emphasized that if avoided, the manifested sequence of events that an archetype produces can lead to neurotic and psychotic disorders (Jung, 1969/1980; Neuman, 1954/1999).

Jung concludes that the challenge of understanding the archetype is that the symbolic process is "an experience in images and of images" (Jung, 1959a/1980, p. 930). Jung explains that the outer experience of the archetype is composed via a rhythm of negative and positive, loss and gain, dark and light. This Jung referenced with the principle of opposites, noted on p.12. For example, Jung's experience of his parents was reinforced through emotions of distrust and confusion about love towards his mother, and weakness and powerlessness towards his father (Jung, 1963/1990). This was later transformed within Jung as he notes in his autobiography:

"That is the handicap I started off with. Later, these early impressions were revised: I have trusted men friends and been disappointed by them, and I have mistrusted woman and was not disappointed" (Jung, 1963/1990, p. 21).

The manifestation of the archetype on the individual is always strongest when consciousness is weakest, limited, and where fantasy can invade the logic of the outer world. This condition is most present when the child is in the first years of life creating a reality that is mostly fantasy based (Jung, 1959a/1980). Shaping them into a pre-existing psychic image, the fantasy image is now intact and influences the sensory stimuli of the child. Interpreted through the observed inherited instincts and preformed patterns of the child, Jung notes that the aptitudes of the child act as a priori to formal conditions based on instinct (Jung, 1959a/1980). It is their presence that gifts the child and the child dreamer its anthropomorphic base of the archetype. Jung continues to hypothesize that the archetype allows for possibilities to emerge as they are not “inherited ideas rather inherited possibilities of ideas" (Jung, 1959a/1980, p. 1448). This 
manifests differently in each individual. For example, the archetypes of light and dark are concomitant upon the geographical location one lives in. Extremes of these archetypal processes are reinforced by living in either southern or northern areas, where light and dark are experienced in excess.

Archetypes are authentic symbols that cannot be excused as signs or allegories, are ambiguous, full of half-glimpsed meanings, and are unfailing in their approach towards us (Jung, 1959a/1980). Thus, integration occurs through a higher level of awareness resulting in the overcoming of the event, as Jung explained about his parents. In the process of time it can evolve over months and years or be played out in a dream and depends solely on the situation at hand, the person it represents, and the goal to be achieved (Jung, 1959a/1980). It is important to note that the universal element to the collective unconscious relies on the notion that, despite the images of the archetypes differing in appearance, it is the intention or tendency of the image that makes it universal in origin (Wolf, 1994). The struggle of understanding the archetype occurs because the discriminating aspect of our nature attempts to establish a single meaning out of them. To Jung the one thing that is consistent with their nature is their manifold meaning, offering us a limitless wealth of reference, making any unilateral formulation impossible (Jung, 1959a/1980).

Now that an understanding of the unconscious, the collective unconscious and the archetypes has been outlined, what follows now are descriptions of some of the common archetypes Jung became aware of throughout his career. They include: The Mother, The Anima, The Animus, The Shadow, The Wounded Healer, and The Self.

\section{The Mother Archetype}

The mother archetype is one of the most powerful experiences during childhood and continues to influence each person's relationship to the world, society and one another 
throughout life (Newman, 1954/1999). In Figure 1 the mother archetype has been interpreted based on Jung's account of his mother in Memories, Dreams, Reflections (Jung, 1961/1990). Being the first significant person to the infant, the mother becomes the constant in the infant's formative years. Two main elements of the mother archetype exist: the maternal and the transformative. Reflected in the principle of opposites, this archetypal process in particular is crucial to the development of the individual (Neuman, 1954/1999). The maternal aspect, therefore, is first experienced by the infant (Jacobson, 1993). Jacobson notes during the first stages of the maternal, no conscious awareness exists, with no independence of the ego from the unconscious. During this time, the mother's role is to nurture, feed and protect the infant, making the infant completely dependent on her. Dependency on the mother establishes security, with the mother figure resembling stability. However, for the male child it can turn into a seduction, and thus needs to disengage to develop his own ego and conscious awareness (Jacobson, 1993). If not, the male may be prone to finding women who will take care of him, being unable to care for himself. Similarly, the female child may have an over exaggeration of all female instincts regarding the male as a procreator seeing him as an object to be looked after.

The transformative, or multifaceted aspect sees both male and female independent and conscious (Jung 1959a/1980). This occurs when the person forms an understanding of the world through their own observations, thus distinguishing themselves from that of their mother's (Jacobi, 1942/1999). Although the archetype of the mother still exists within, it informs their lives, rather than impedes it. For example, in the male an openness can develop, giving him the capacity for friendships with both sexes, possessing gifts such as teaching and love of history and religious sentiment (Jung, 1959a/1980).

\section{The Divine Syzygy}

During the formative years of childhood, the archetypal form of the divine syzygy (anima 
and animus) takes shape and acts like a cover assimilating the image of the real parents until, with increasing consciousness, the true figures of the parents are perceived. The term 'syzygy' comes from Valentinian cosmology describing a pair of corresponding qualities known as 'aeons' that combine to create a state of wholeness (Smith, 2009). Much like the yin/yang symbol of Chinese traditional medicine the syzygy forms the basis of the female (anima) and male (animus) polarities in the individual. Thus, the emotionally charged content of the syzygy motif has both a masculine aspect and a feminine one. Albeit, these traits are most prominent in the actions of the opposite sex; in man the feminine traits, and in woman the masculine (Jung, 1959a/1980).

\section{The Soul-Image}

The archetypal figure of the soul-image is what Jung referred to as the respective contrasexual aspects of the psyche. Known as the anima for the male and the animus for the female, it is the image of the other sex that is carried within us, showing how the human experience of the opposite sex is created. As Jung has maintained, everything latent, unexperienced and undifferentiated in the psyche remains in the unconscious with the man's 'Eve'(anima) and the woman's 'Adam' (animus) being projected onto the experiences of the opposite sex. The challenge to each person comes with the understanding of the dream content reflected by the anima or animus and especially in outer reality with the projections of the unconscious aspect onto the opposite sex occurring from each person onto themselves. In other words, we fail to understand that our unconscious is providing us with this experience rather than it being done to us by another person (Jacobi, 1942/1999).

The characters that reveal themselves to us from the soul-image depend upon our innate psychological disposition, mirroring where we are in our own personal development. Thus, the multitude of forms in which the soul-image can appear are numerous. They are rarely explicit, 
complex in nature, outfitted to reveal the contradictory nature of the individual (Jacobi, 1942/1999).

\section{The Anima}

Being the unconscious feminine aspect to the male psyche, the anima is the compensatory aspect to masculine consciousness. Once the male has successfully formed his own ego, separate yet also influenced by the mother archetypal experience, his anima begins to develop. Neuman refers to this as the crystallization of the anima from the mother archetype. This union is complete whereby the adult male combines with a feminine partner of similar age and characteristic (Neuman, 1954/1999). The opportunity thus presents itself when the male is able to deduce the workings of the anima both in his waking and his dream life. Therefore, the male's relationship to the anima develops and his interpretation of it, is reliant on his understanding of the role the anima has (Neuman, 1954/1999).

Jung notes that the anima resembles the muse bringing men face to face with their pleasure principle (Jung, 1959a/1980). Originally theorized by Freud, the pleasure principle is an instinctual attempt to avoid pain and to satisfy primordial biological urges (Berzoff, Melano, Flanagan \& Hertz, 2008). For Jung, the anima acts as a priori element to the male's moods, reactions, impulses and spontaneity of life (Jung, 1959a/1980). Thus, the anima can be cunning, convincing, and ruthless. At first glance the anima is dominant, operating unknowingly, in an unpredictable nature. It possesses a seductive presence that excites the male's senses, having the potential to lead them astray into the illusionary traps she lays.

Despite this, Jung urges us to consider the purpose that it serves. Noting that a depth of awareness surrounds her, a wisdom yet to be uncovered, Jung concludes that this truth reveals itself only when the individual has regarded the anima as the significant aspect to development. Jung notes that once recognized, the anima not only reveals a deeper meaning to man, but an 
understanding that reflects a higher comprehension to life's laws (Jung, 1959a/1980). This deeper meaning lies in the understanding of the anima's nature. This is revealed through the principle of opposites, for the dark aspect of the anima helps man understand his own nature and his response to it determines his understanding of the female aspect inside of him. This occurs when the male surrenders initiating defeat, having man face his fear of demoralization. Once interpreted, the anima loses the power she has. Amidst the chaos there is an order to things and Jung notes that the interpretation of the anima emerges when we surrender to the greater picture. This surrender allows for a disabling of the reality we thought was there into a reality that has always been there. (Jung, 1959a/1980).

\section{The Animus}

Neuman (1954/1999) emphasizes that the "numinous father-husband image" (p. 172) of the animus, known to humanity through the cultural understanding of God/Creator, has been imparted onto the unconscious experience of woman. Thus, the animus is the unconscious male aspect of the female unconscious. Neuman continues to note that cultural values find its roots in its fathers, who represents and reinforce the religious, ethical, political, and social structure of the society. The fathers supervise the education of society, protect masculinity, and preside over the upbringing of the individual to certify its coming of age. Finally, Neuman stresses that cultural values of the "numinous father-husband image" (p. 172) exist worldwide from indigenous peoples to that of a Christian society suggesting that the animus is a universal archetype existing across humanity (Neuman, 1954/1999).

Whereas the anima is unpredictable and moody, the animus is rigid, relying on principle and remaining loyal to its creed (Jung, 1959a/1980). Its role is to establish order, logic and direction. The animus is influenced by mediocrity and reinforces the law. It is dogmatic as it is theoretical and can become defensive and domineering( Jacobi, 1942/1999). Jacobi notes that the 
female's goal with respect to the animus is to overcome a lack of self-confidence and inert resistance. As with the anima, the principle of opposites comes into being with the higher and lower figure of the animus presenting itself to the female. Thus, understanding the motivations of the animus is critical in the female's development. This is presented to the female in outer reality where qualities of the animus are projected onto a real man, or in dream imagery from the collective unconscious (Jacobi, 1942/1999).

\section{The Shadow}

The process of integrating archetypes, through the process of individuation (described on p.35) often begins with the realization of what Jung refers to as the shadow (Jung, 1959b/1980). Integration of the archetypes is important as it leads to the emergence of the self, the archetype that defines our true nature, an overall integration of all archetypes and their processes (Jacobi, 1942/1999).

The shadow is the figure that appears in many personified forms. Being part of the individual, it is split off from conscious awareness. Often expressed through dream symbolism, the shadow is dialectical in nature and often dramatic in its manifestation (Sandner \& Wong, 1997). The meeting of the shadow oftentimes coincides with conscious understanding of the functional and attitudinal aspect of ourselves that we reject on grounds of morality, religion etc. Its suppression is due to its contradicting nature to our own conscious principles. Also occurring in our outer reality as concrete forms, the shadow can manifest as an encounter with a situation where our projections become revealed to us. Thus, this figure of the unconscious is strong, manifesting itself in our reactions to others (Jacobi, 1944/1999)

The shadow is often the same sex of the individual (Sandner and Wong, 1997) yearning to be part of the personality structure, bringing great challenges to the individual and his life (Jung, 1959a/1980). The shadow is a necessary step in the understanding of oneself that no one is 
exempt from. These encounters are necessary acts by the unconscious that force a meeting with the hidden aspects of our personality. If one chooses not to understand the nature of himself then he becomes unconscious of himself, haphazardly living life as reactions to events rather than using life's events as reflections to make himself anew. Since the unconscious is mainly influenced by the archetype of the shadow, it is also the most accessible and easiest to experience of the archetypes (Jung, 1959b/1980).

The shadow allows us to know ourselves, good and bad, showing us both sides of our nature. It is as creative as it is spontaneous, representing our animal instincts. Acting as a mirror the shadow shows us our own inadequacy (Jung 1959b/1980), therefore being destructive and painful to the ego (Guggenbühl-Craig, 1971). If one can see the influence of his own shadow Jung suggests a small part of understanding our own nature has been resolved as we have brought up aspects of the personal unconscious to our awareness (Jung, 1959b/1980).

One individual whose shadow experience has caught academic interest is the shaman. Sandner and Wong (1997) note that due to the encounter with his own shadow, the shaman possesses an ability to penetrate the depths of the soul to circumvent disease. As well, this experience offers the potential for psychic balance, enhanced consciousness and spiritual power (Sandner \& Wong, 1997).

\section{The Wounded Healer Archetype}

Often being misunderstood, discounted, or romanticized, the wounded healer archetype has received minimal academic attention (Zerubavel \& Wright, 2012). Drawing from Greek mythology (Jackson, 1992; Kirmayer, 2003), Jung was the first to reference the archetype of the wounded healer from a psychotherapeutic perspective. Examples of the wounded healer archetype include Greek mythological figures such as Apollo, Chiron and Asclepius (Dunning, 2006). Representing enlightenment and healing, Apollo sought out objectivity and intellectual 
pursuits. The half-man, half-horse Chiron, and leader of the Centaurs was known for his knowledge of healing through medicine, and Asclepius, the son of Apollo was trained by Chiron and later became known as superlative healer (Dunning, 2006).

Images of the wounded healer permeate religion, philosophy, and art, but also have a place in healing traditions such as shamanism suggesting that the construct of the wounded healer has existed for over 2500 years (Kirmayer, 2003). To appreciate the domain of the healer, Kenny (2012) suggests that an acceptance of a dimension of being that exists beyond the physical and material and everyday consciousness is required. Although his early writings indicate that the wounds of therapist act like a contamination that must be eliminated, Jung's writings later shifted to reveal that "only the wounded physician heals" (Jung et al., 1964, p. 134). Thus, it is not surprising that many psychotherapists arrive at their profession through a journey that involves a history of pain or suffering (Barnett, 2007; Farber, Manevich, Metzger, \& Saypol, 2005). Guggenbühl-Craig (1999) extended Jung's theory of "the wounded physician" by highlighting that as soon as the "inner healer" was activated in the patient by the analyst then the patient transforms from sufferer to healer. This builds on Jung's reflection that the patient acts as the antidote for the doctor's wound (Jung, 1963/1990). Reflected in Figure 2 below, the Psychotherapist transfers the intention of healing onto the client who then integrates this understanding allowing for the archetype to constellate into the patient's consciousness. 


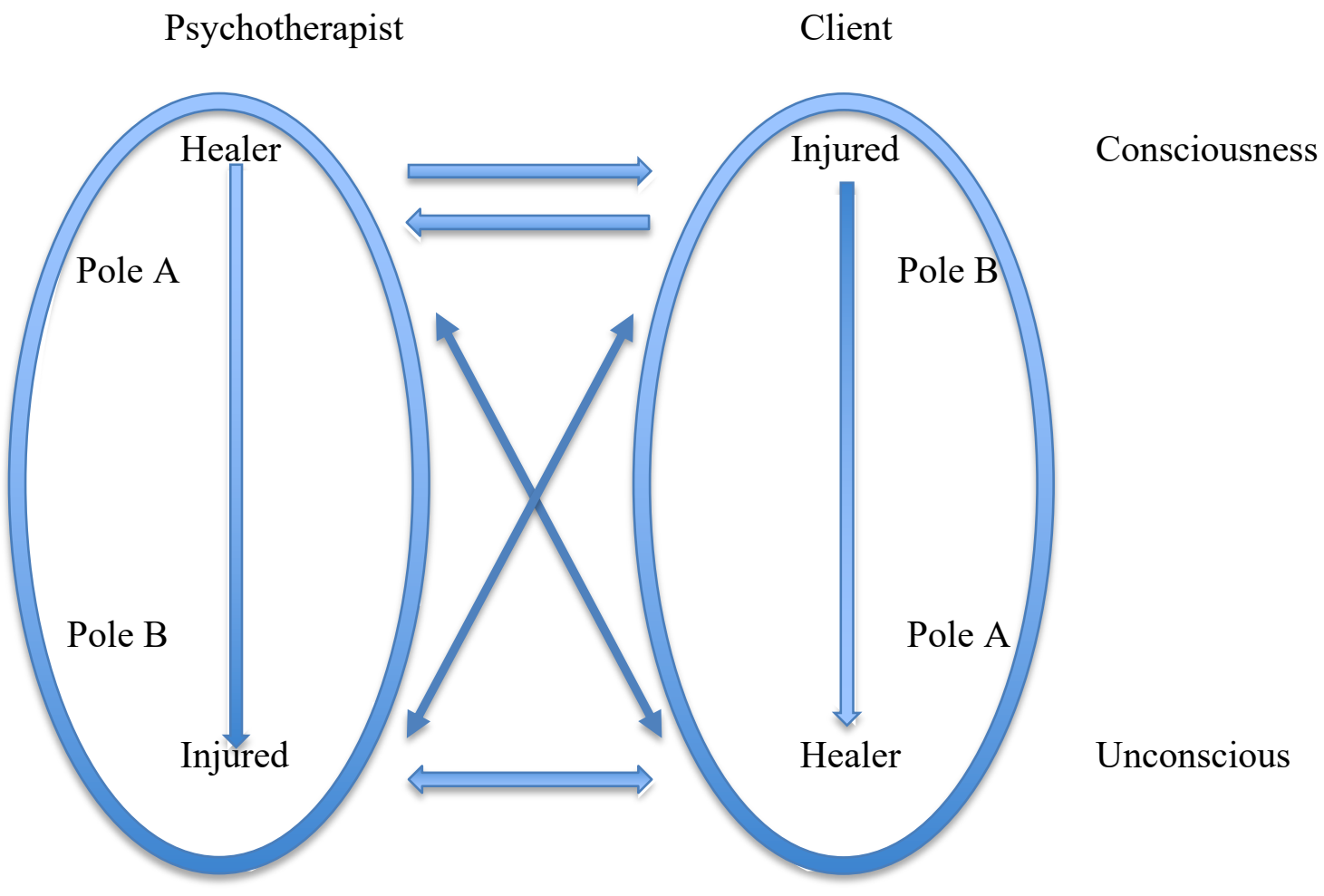

Figure 2. Adapted from Chalverat, C. (2000). The Figure has been translated from French to English and depicts the archetypal process from being wounded to being healed. Copyright has been granted by Chalverat, C. Please see Appendix A for the details of the permission.

Few detailed reports exist about what it means for a psychotherapist to process, resolve, or recover from a wound in such a way that it might encourage effective psychotherapy (Dunning, 2006). Freshwater (1995) suggests that healing and suffering cannot occur without each other and are unified opposites, noting that both are required to achieve harmony. Jung would argue that this is a necessary aspect of the individuation process (please see p. 35), where both polarities of the archetype are integrated (Jung, 1959a/1980). Derived from the old English word 'hal', to make whole, 'healing' is often referred to as the harmony of the physical, psychological, spiritual and social aspects of ourselves (Keegan, 1994). Indeed, it is the effective understanding of what suffering teaches that allows the wounded healer growth and transcendence of their own 
wound to be able to integrate their experiences into healing relationships. (Conti-O'Hare, 2002).

Zerubavel \& Wright (2012) propose that the significance of a healer's woundedness varies on two dimensions: (1) characteristics of the wound itself, i.e., relevant stigma, social taboos, and (2) the scar that the healer bears, i.e., how marked the healer is by the wound and how fully recovered the healer is. Both aspects are critical to understanding psychotherapists' varied response to the wounded healer archetype. The authors also indicate that recovery is neither linear or, when achieved, permanent. Consequently, uncertainty about the wounded healer's future course is indefinite with the wounded healer never being fully healed (Zerubavel \& Writght, 2012). Further to the dimensions above, the authors propose four different types of future trajectories that may help characterize psychotherapists who have struggled with a significant wound: (1) a trajectory anticipating recovery over time; (2) a trajectory characterized by posttraumatic growth; (3) a relapse trajectory, anticipating fluctuations and setbacks; and (4) a chronic dysfunction trajectory anticipating continuous symptom-related struggles (Zerubavel \& Wright, 2012). Thus, if the archetypal notion of healing through the wound is properly channeled, some therapists will enable their own woundedness to promote recovery in clients.

Henri Nouwen's account of the wounded minister in Christian society raises an important point as he concludes the wounds of the minister have to bear witness to the sufferings of the individual. His account discusses the confusion and loneliness of the modern world where sacredness is difficult to see, with faith having to be reclaimed. Using hope as his binding force, Nouwen concludes that the minister's call is to be both wounded and healed yielding to the pains that modern individuals experience. Thus, the minister has to have his own experience of pain, suffering, loneliness and confusion to circumvent the wounds he comes across (Nouwen, 1972). Becoming a catalyst for healing within the client, the wounded healer's dualistic forces of woundedness and healing begin to take root (Miller \& Baldwin, 1987; Nouwen, 1972; Sedgwick, 
1994). Focusing on the concept of the wounded healer from a pastoral counselling perspective, Means (2002) asserts being wounded by personal life experience allows the counsellor to work from an "empathic bridge" enabling the counsellor to understand the client's feelings of guilt, shame and disappointment.

Central to the theme of woundedness are the phenomena of transference and countertransference. To Jung, transference is a type of projection. From a psychotherapeutic experience, projection is an unconscious, automatic process whereby the information that is unconscious to the subject is transferred to an object (person), so that it appears to belong to that object. During a psychotherapy session, this occurs in the transference between client and psychotherapist. Jung noted that both are part of a dyad with transference being the unconscious redirection of emotions from one person to another and countertransference being the response by the psychotherapist of that projection (Jung, 1963/1990). The countertransference response by the psychotherapist is achieved by offering an understanding of the unconscious meaning of the transference to the patient (Knox, 2003). Thus, the subjective experience of the wound allows for understanding to occur, and the potential for healing to manifest in the patient (Merchant, 2012). Even if the countertransference response is not shared with the client, Sedgwick (1994) suggests that the therapist's ability to decipher the countertransference allows for the possibility for therapy to proceed.

To Jung, countertransference is also a type of projection. Thus, the healer's woundedness can act as an internal reference point for understanding a client's pain (Hayes, 2002). Furthermore, the therapist's appropriate use of self-disclosure (personal experience) may also instill hope of healing and recovery (Kirmayer, 2003; Miller \& Baldwin, 1987). For example, the College of Registered Psychotherapists of Ontario (CRPO) emphasizes that the safe and effective 
use of self in psychotherapy is one of the principal competencies a psychotherapist must embody. This entails an understanding of one's own subjective experience and dynamics of interaction that contribute to the psychotherapeutic process (www.CRPO.ca). Finally, working as a psychotherapist can be a healing and restorative experience (Zerubavel \& Wright, 2012). The pain the psychotherapist carries and how it is used to help clients challenge the notion that psychotherapy is a learned process as the internal understanding of the wound manifests to help both the therapist and the client (Barnett 2007). Please see Figure 2 on p.31 to gain a visual understand of the process.

Despite the advances in psychotherapy in the application of the wounded healer, confusion exists concerning an adequate definition of healing in medicine (Egnew, 2005). This is contrary to the nursing literature noting that healing involves bringing together aspects of one's self, body-mind-spirit, at deeper levels of inner awareness, allowing for integration and balance to occur with each having equal importance and value (Dossey, Keegan \& Guzetta, 2005). Offering no operational definition of healing, the field of medicine offers no explanation of its mechanisms, except explanations of narrow physiological processes concomitant with curing disease (Dossey, 1995). Egnew (2005) concludes that the MEDLINE electronic database has no heading for "healing" and instead offers qualifiers associated with the spiritual and religious aspects of illness and recovery associated to psychology and alternative medicine. Egnew (2005) further notes that if healing is at the core function of medicine, then investigation of its symbolic meaning obliges research to consider healing phenomena (Kleinman, 1973), along with an operational definition of holistic healing to be established (Egnew, 2005). Arguably, the notion of healing in psychotherapy and medicine is the impetus for both disciplines' existence. With the archetype of the wounded healer being paramount in both areas, whether it be a psychotherapist or doctor the intention to offer healing is paramount. 


\section{The Self and the Individuation Process}

Now that a detailed understanding of Jung's psychology has been presented, we now turn our attention to the Individuation Process, where the archetype of the Self is realized. As noted in Figure 1 (p.13) the self exists alongside the archetypes, appearing to be surrounded by them. In Jung's psychology, the Self was his No. 2, the God-image archetype associating with the collective unconscious (Jung, 1963/1990). Using alchemy as a metaphor to describe this experience, Jung notes the individual becomes conscious of his own psychological process, thus transforming himself into a conscious individual (Jung, 1963/1990). Consequently, it is the structure of Jung's psychology that lays the foundation for the Individuation process to occur. This is achieved through the recognition and constellation of the archetypal process, allowing for the archetype of the Self to emerge.

Schmidt (2008) notes that Jungian psychology understands the self as a unification of body and mind, the union of opposites and the force that propels the individual on his/her journey throughout life. As Jung notes, "it is this archetype from which we can no longer distinguish the God-image empirically (Jung, 1963, p. 476).

As mentioned previously, the self is what the ego develops from (Schmidt, 2008). Neuman $(1954 / 1999)$ mentions that the unconscious activity of the self dominates the entirety of our lives, however it is during the second half that this becomes conscious. During the first half of life there is an outer expansion or focus by the ego. As it forms in our childhood, it is during puberty where it centers itself in consciousness being unconsciously informed by the self. Molding itself into consciousness, the ego begins its journey adapting to the personal achievements of adult life such as relationships, power or creative work. This personal focus begins to shift during the second half of life where this process is reversed, and emphasis becomes between the ego and the collective unconscious (Neuman, 1954/1999). Consequently, the responsibility of the self is 
to integrate into conscious awareness the archetypes for the amalgamation of the whole personality to occur (Stevens, 1994). This integration process takes the ego back along the path it took during the first half of life, and the goal is a synthesis between the ego and the self. As a comparison, Jung mentions the mystical transformative experience of the shaman, concluding that the approach the shaman takes is a vital step towards achieving "his true personality" (Jung, 1967/1970, p. 277). Drawing from research in Siberian shamanism, Jung uses the symbol of the tree as the vessel that the shaman climbs to find his true nature in the skies above (Jung, 1967/1970).

In his writings, Jung reflected that personal transformation and growth extended past childhood and adolescents through mid-life and into old age (Stevens, 1994). Jung (1959a/1980) refers to individuation as the "process by which a person becomes a psychological "in-dividual", that is a separate, indivisible unity or whole" (p. 5113). During individuation, the psyche matures through the self-regulation process that the ego undertakes reaching consciousness of the self. This growing realization allows for the self to constellate out of the unconscious becoming a conscious aspect of the personality. An assimilation begins to occur, whereby the integration of the complexes and archetypes begins to take shape (Neuman, 1954/1999). Neuman suggests that individuation can occur without damaging the integrity of consciousness, however challenges in integrating archetypes arise when the ego perceives the archetypes as an invasion.

Jung's theory of the psyche offers humanity a possibility towards wholeness. This wholeness is achieved by the successful completion of the individuation process (Jung, 1963/1990). It begins unconsciously, in our own ignorance, firstly understood by our identification with our gender role (Jacobi, 1942/1999). Although depth psychology has taken more of an androcentric view, placing males at the pinnacle of success, health and wealth, Jung's view of gender attempted otherwise (Young-Eisentdrath \& Dawson, 1997). Young-Eisendrath 
and Dawson (1997) note that Jung's view offered one vital characteristic, "the opposite sex as a projection-making factor" (p.224). Indeed, Jung's archetypes of the anima and animus are not only culturally observed but also act as projections. His offer to help us see characteristics of ourselves through our projection into others, has countered our ignorance and brought insight into our unconscious other. This has sparked an essentialist debate as descriptions of the anima and animus have thought to be restrictive as Jung has attributed common characteristics to the male and female unconscious other ('lolana, 2016). For example, 'lolana (2016) suggests Jung saw all women as illogical due to them not having a Logos-centred approach to the attainment of knowledge and if they did, was due to a misunderstanding within family dynamics (Jung, 1971).

Despite the limitations that Jung may have had towards gender development, Jung is certain about its psychological manifestation. The outcome is the awareness of the unconscious other within, a subpersonality that takes on a life of its own, often dissociated and projected onto the opposite sex. The feminine subpersonality of the male seen in the anima, and the masculine subpersonality seen in the female as animus. Both are thought to be biologically driven, occurring naturally throughout the lifetime of the person (Neuman, 1954/1999). Despite the essentialist tone, Jung's contrasexual view contributes to depth psychology helping us see that the dark aspect of the subpersonality belongs to its owner (Young-Eisentdrath \& Dawson, 1997).

We now turn our attention to the shaman, who according to Jung has become conscious of the unconscious other within, thus achieving the individuation process. 


\section{CHAPTER III}

\section{WHAT IS A SHAMAN?}

Let us now investigate the relationship between the concept of the wounded healer archetype and the shaman. An overview of the relevant research on shamanism will be presented first by highlighting the work of Eliade (1964/2004); VanPool (2009); Walsh (1989; 1994; 2007) Krippner (2002; 2012), and Winkelman (1984; 1989; 2010).

Research on shamanism from western scholars has been occurring since the eighteenth century (Flaherty, 1992) with literature on the subject spanning the fields of anthropology, archeology, comparative religion, history, psychology and neuroscience (Sidky, 2010). Traditional shamanism was practiced in small, autonomous societies with the shaman serving his/her community (Eliade, 1964/2004; Krippner, 2012; Walsh, 2007; Winkelman, 2010). However, recent shifts in practices due to the modern world's influence has caused worldwide accessibility challenging the idea of what a shaman is. Although recent popularity on shamanism has been attributed through the mystical attraction it has, it has now established itself alongside postmodernism through its New Age appeal. With postmodernism comes skepticism, subjectivism and a general distrust of how Western thought has been established with ideological principles being used to maintain order (britanica.com). Following this, popularity with shamanism has been advanced by the New Age movement that focuses on esotericism, a religious outlook based on the application of mystical knowledge through personal growth and healing (britanica.com). As a result, it has caused confusion amongst scholars due to the basic epistemological principles required for quality scientific research. Thus, non-scholarly applications have led to mainstream use of the term, removing the essence of what shamanism is, resulting in misuse and confusion (Hamayon, 2001). 
Most scholars would agree that it was Eliade's (1964/2004) cross-cultural ethnographic study that initially revealed the importance of shamanism (Winkelman, 2010, Hulkrantz, 1985). Spanning four of the six continents of the world: (1) North America, (2) South America, (3) Europe/Asia, and (4) Australia/Oceania, Eliade concluded the shaman is both a medicine person and a magician. Referring to it as a religious phenomenon of Siberia and Central Asia, Eliade's ideas about shamanism placed it into mainstream thought, giving it a new tone, validity, and importance (Sidky, 2010). Originating from the Tungus term 'saman', Eliade defined the shaman as utilizing a specific type of "magic" that included "mastery over fire" and "magical flight" (Eliade, 1964/2004, p. 5). According to Sidky (2010), Eliade's work romanticized the shaman persona into scholarly research (Sidky, 2010). Eliade (1964/2004) concluded that the shaman used these "magico-religious powers" (p. 3) and entered into a unique state called the "the archaic technique of ecstasy" (p. 74) to effectively communicate with the celestial Supreme Being. Once in contact with this supernatural entity, the shaman obtained information on healing, spirit interaction, defeating demonic beings, and the ability to escort souls to their destination (Sidky, 2010).

Eliade (1964/2004) notes that becoming a shaman can occur through hereditary transmission, a spontaneous vocation, or by being named by the group that the shaman represents. Regardless of how an individual becomes a shaman, the process of transformation that it requires is known in the literature as the death and rebirth process. This will be described in detail in the section concerning the shaman as a wounded healer. For now, the death and rebirth process is the process whereby the shaman initiate has a psychological experience of transformation whereby the shedding of the old self is replaced with the characteristics of the shaman (Walsh, 2007; Winkelman, 2010). 
According to Eliade (1964/2004), the shaman becomes anointed when four types of teaching have been received: (1) ecstatic knowledge (acquired information that the shaman obtains while immersed in the ecstatic state) attained through dreams and trances and by traditional means through shamanic techniques, (2) names and functions of spirits, (3) mythology, and (4) genealogy of the group the shaman represents. Equivalent to an initiation process, this instruction is handed down by master shamans and spirits of ancestors who are the primary keepers of this information. The ecstatic experiences the shaman has is maintained and strengthened by the religious ideology and mythological foundation from the culture the shaman represents (Eliade, 1964/2004). According to Eliade, the shaman achieves this by employing a specific trance enabling the shaman to leave his body and ascend into the sky (upper world) or descend into the underworld. The upper world contains teachers or spirit guides whom the shaman interacts with to find help and guidance whereas the lower world contains power animals from whom the shaman acquires the power needed to help the individual or community (Shepard, 1999). Eliade maintained that the abilities of the shaman are a universal attribute to all of humanity, being used by cultures across the world (Sidky, 2010).

The ecstatic trance experience outlined by Eliade (1964/2004) has become known in the literature as the altered state of consciousness (ASC) that the shaman uses willingly to help the people in his/her care (VanPool, 2009). Being erroneously associated with the Western definition of trance in psychopathology, anthropology has attempted to compare and contrast this phenomenon (Bourguignon, 1989; Winkelman, 1986). Despite research arguing over the terminology and definition of the ASC (Rouget, 1985, as cited in Bourguignon, 1989) the critical defining aspect of the shaman is his/her ability to self-induce the ASC and leave it at will (Peter \& Price Williams, 1980). As this is the present study's focus, the ASC will be discussed at length in Chapter 4. 


\section{Defining Shamanism}

Eliade's (1964/2004) work began a movement in the study of shamanism and is still referred to today by notable researchers in the field (Hulkrantz, 1989; Winkelman, 2010, Walsh, 2007). However, as research on the shaman continued, Eliade's work was criticized (Kehoe, 1996) as it took an etic (observing and understanding a culture from an outside perspective; Webster Dictionary online, n.d.) approach to shamanism classifying it by a reductionist framework based on specific behavioral characteristics of the Siberian Tungus tribe (Jones, 2006). Sidky (2010) argues that by singling out the magico-religious beliefs of the indigenous people of Siberia and other regions depicts the shaman as "living fossils" (p. 9), conveniently forgetting the influence of other cultures such as what the Soviet regime had done at the time. As well, Sidky (2010) argues that Eliade's interpretation of the records of travelers between the sixteenth and nineteenth centuries that described the account of the shaman was a, "cut and paste job" (p.10), as Eliade selected qualities and characteristics that were out of context. Finally, Sidky (2010) points out that cultures present at the time of Eliade's work differed to that of the historical picture presented of the Paleolithic culture where shamanism came from, with ecological, economic and social conditions in Siberia being vastly different from what Eliade suggested.

As research on shamanism evolved, it began to compare the religious structure of the West to the practices of the shaman (VanPool, 2009). As a result, research identified the shaman as a religious practitioner placing the shaman alongside the priest (VanPool, 2009). Disagreeing with this idea, VanPool (2009) notes that what is necessary is that research considers the variations seen in shamanism across culture while also realizing that the shaman's use of the ASC is only the first step towards understanding the cultural belief system from where the shaman originates. VanPool (2009) concludes that specific historical and cultural practices have 
provided clear cross-cultural similarities and differences between shamans and priests. For example, VanPool (2009) contrasts the written word of the bible against the shaman's use of iconography, anthropomorphic figures, tutelary creatures (guardian spirits) and tools (pipes, drums, crystals) as identifying with the divine. Keeping this in mind, Van Pool (2009) suggests that using a general methodology for identifying the presence of shamanism as a religious system and synthesizing the relevant characteristics of the shamanic experience is required.

Criticizing this, Vitebsky (1995) suggests that shamanism be viewed from a crosscultural perspective, noting its variability of practice and approaching it as a form of religious sensibility. Furthermore, Bowie (2000) concludes that a shaman is a religious specialist existing within many frameworks who employs techniques within a specific belief system. Bowie (2000) concludes that the term encompasses an array of different phenomena, having little resemblance to one another.

Walsh's (2007) research on shamanism has offered science a broader focus, offering a social description of what shamanism is. In his most recent work: The World of Shamanism: New Views on an Ancient Tradition, he indicates that the phenomenology of shamanism encompasses a historical, cross-cultural and spiritual focus. Noting that death and rebirth is common to the shamanic crisis of transformation, Walsh (2007) notes that the process can involve "considerable stress and even reach psychotic proportions" (p.111). He explains this process of death and rebirth also occurs throughout the world's culture and religions, suggesting similar transformative processes exist. Thus, Walsh (2007) notes that research can now compare shamanism to other disciplines, such as yoga and contemplative meditation. The spiritual focus of the shaman identified by Walsh incorporates what he calls the seven central spiritual practices: (1) living ethically, (2) transforming emotions, (3) redirecting motivation, (4) training attention, (5) refining awareness, (6) cultivating wisdom, and (7) serving others (Walsh, 2007). 
Similarities between the shaman healing traditions that alter consciousness are also found in Eastern meditation traditions. These include repetitive chanting, percussion and music, fasting, sexual restrictions, sensory isolation and austerities as well as ritual activities focusing on occupying attention (Winkelman, 2010). Although meditative traditions focus on the direct approach to parasympathetic dominant states, traditions like those of the Islamic mystics who are known for the whirling dervishes using sympathetic stimulation as the root to achieve this. An important distinction between meditative traditions and the shaman's use of the ASC is that the shaman is in a direct communication with what is encountered in the ASC, experiencing it as a non-ordinary realty whereas meditators focus on self-control, lowering arousal, experiencing a sense of calm, emotional detachment, loss of sense of self, and an expanded awareness (Winkelman, 2010).

Further to his description of shamanism, Walsh $(1989,2007)$ suggests that shamanism is one of humanity's earliest examples of a healing tradition involving psychotherapeutic and ritualistic methods. Offering some key distinguishing features towards his definition of shamanism, he defines shamanism as a family of traditions that use the ASC willingly. In addition, Walsh $(1989 ; 1990)$ mentions that shamans experience themselves as a soul or spirit travelling (known as soul flight, spirit journey, cosmic journeying) to other realms and interacting with spirits to serve their community needs.

Walsh (1994) suggests that the terms soul flight, spirit journey and cosmic journeying in the literature are all synonymous with the out of body experience. Being able to interact and understand the spirit's intention, the shaman uses the information to facilitate healing in the client or community he or she is helping. Finally, when considering the term spirit, Walsh (2007) suggests that it not be interpreted as literal and be understood as what the shaman is experiencing according to the specific cultural framework being used. 
Stanley Krippner $(2002 ; 2012)$ notes that the existence of shamanism across culture suggests an adaptive ability to the evolving needs of society. Acknowledging that the shaman initiate experiences a process of transformation whereby a physical deconstruction occurs in dreams and visions, Krippner (2002) mentions it results in "the reconstruction of bones and flesh, during which there is an ecstatic birth" (p. 969).

In support of Eliade (1964/2004), Krippner (2002) acknowledges that the defining feature of the shaman is the use of the ASC to achieve soul flight/ecstatic journeying or an out of body experience. Furthermore, this manifests through pretending and role-playing via a direct interaction with the natural environment (Krippner, 2002). Krippner (2002) concludes that shamans are intriguing to science because they represent the marginalized other. Suggesting that trickery, improvisation and unpredictable behavior can occur in the shaman's rituals, Krippner (2002) notes that this adds to the fluidity of the human experience. Krippner (2002) continues to mention that it is a priority for the shaman to share esoteric knowledge with his community by deconstructing the rules of society to allow for change to occur.

Krippner (2002) points out that ritual in shamanic culture is designed to evoke healing and relieve suffering by providing a direct link between the ordinary world and the spirit world. Thus, the symbols, mythology and technology used are specific and purposefully directed (Krippner, 2002). In his reflections he hypothesizes that shamanic epistemology uses perceptual, cognitive, affective and somatic methods that assisted early humans to survive. Thus, Krippner (2002) concludes that the shaman may have been the original specialist using: (1) healing, (2) storytelling, (3) divination, and (4) rituals to achieve specific results. Accomplished through focus and control over the ASC, the shaman is a practitioner who can self-regulate his "bodily functions and attentional states" (Krippner, 2002, p. 2) to obtain information that is unavailable to the individual(s) who has come for healing. 
Krippner (2012) mentions that many traditional healing systems do not separate the body and mind into parts such as the West does with doctor's having a specific focus on psychology, brain surgery, heart surgery, etc. Rather, Krippner (2012) mentions that shamanism views the human as one complete organism existing as part of an external system that may have caused the illness to manifest. From this, the shaman can divide illness into biological and psychological categories and those of metaphysical causation. Thus, the shaman may visit the individual's family or tribe to properly diagnose the illness. Identifying factors that are also recognized include: (1) age, (2) gender, (3) social position, and (4) belief system as possible causes (Krippner, 2012). For example, Krippner (2012) highlights the Navajo practitioners (hataalii) who have identified three main diagnostic criteria of mental illness: (1) "moth craziness" (identified by fits of uncontrolled behavior such as jumping into a fire like a moth), (2) convulsions, rage and violent behavior (the most prominent of them all), and (3) "crazy violence" mainly due to alcoholism but also other external manifestations (Krippner, 2012, p. 74).

In his cross-cultural ethnographic study on magico-religious practitioners (those healing practitioners who psychologically induce trance states), Winkelman (1984) observed 47 societies identifying several categories including: (1) shamanistic healers, (2) shamans, (3) shaman/healers, (4) healers, (5) mediums, (6) priests, (7) witches, and (8) sorcerer/witch. Based on coding data from ethnographies and quantitative analysis, Winkelman (2010) expanded on Eliade's (1964/2004) definition of shamanism and determined the etic and cross-cultural characteristics of the shaman.

Winkelman's (2010) definition of a shaman includes: (1) a death and rebirth (the process whereby the initiate "dies" and is "birthed" into a shaman), (2) a charismatic leader in hunter gatherer societies who acts with an informal political power being representing by high social 
status, (3) having a leadership role in raiding parties, and an (4) influence over communal hunts and group movement. However, of all the characteristics identified by Winkelman (2010), the defining feature of the shaman is the utilization of the ASC for obtaining relevant healing information for the individual and the group. This information is obtained in the following ways by: (1) soul flight (out of body experience), (2) animal transformation (ability to transform or "shape shift" into animals while induced in the ASC), (3) interaction with animal spirits, and (4) hunting magic (ability to locate game for food). Winkelman (2010) notes that shamans also organize ritual healing and divination activities on behalf of the local community and are also thought to participate in malevolent acts designed to harm others such as enemies of the community. Finally, the ASC of the shaman has helped society evolve and adapt acting as an evolutionary imperative for survival (De Rios \& Winkelman, 1989).

What Winkelman $(1984 ; 2010)$ has revealed to research is that the shaman is a product of a sociocultural evolution of consciousness (Ogembo, 2005). Ogembo's (2005) research on symbolic and interpretive anthropology furthers this argument by looking at shamanism as an internally orientated and intuitive form of consciousness. Basing his understanding of shamanism on Winkelman's (2010) work, Ogembo (2005) argues that more can be understood about the fundamental aspects of consciousness and its relationship between physical and mental levels of reality. Since the shaman is known for utilizing the ASC, Ogembo also supports Winkelman's (2010) need for a description of a structural foundation from where consciousness originates. This will allow for a systems model that links consciousness to its biological base and therefore its experiential orientation.

The work of Michael Harner $(1962,1980 / 1990)$ also deserves attention. His work with Jivaro shamans (1962) and others around the world has contributed to the establishment of the Foundation for Shamanic Studies that offers training in shamanic techniques such as the 
shamanic journey and other shamanic methods. Offered across North America and Europe, the shamanic training program has helped people with self-awareness and personal healing.

In his foundational work, The Way of the Shaman, Harner (1980/1990) describes the shaman as the holder of ancient techniques used to create well-being and healing for themselves and members of their community. Along with Eliade (1964/2004), Krippner (2012),Walsh, (2007), and Winkelman, (2010), Harner (1980/1990) defines the shaman as man or woman who enters an ASC at will to access a hidden reality where knowledge, power and healing are acquired for the client or group requiring help. Also, recognizing similarities across culture, Harner (1980/1990) suggests that the methods employed by the shaman have achieved similar, if not better results than of our medical community. Shamanism is referred to by Harner as "a great mental and emotional adventure" (1980/1990, p. xvii) helping individuals transcend their own perception of illness. Harner notes that the shaman's effectiveness occurs at a deep level of consciousness where the shaman merges the image of the healthy individual with that of the individual's own image to encourage healing to occur. Thus, a deep emotional commitment is formed between the shaman and patient. Finally, the shaman's ability rests with his maintenance of his/her own personal strength and willpower (Harner, 1980/1990).

As has been outlined thus far, research from Eliade (1964/2004), Krippner (2012), Walsh (2007), Winkelman (2010), and Harner (1980/1990) indicates that becoming a shaman requires the individual to move through a psychological transformation known as the death and rebirth process. As well, the researchers agree that the shaman's use of ASC is to help the individual or the community heal. This is often achieved by using rituals to induce the ASC whereby communication with spirits occurs (Harner, 1980/1990; Krippner, 2012; Walsh, 2007; Winkelman, 2010). Further to this, Walsh (2007) has outlined seven central spiritual principles that define the shaman's approach to his/work. Once again, they are: (1) living ethically, (2) 
transforming emotions, (3) redirecting motivation, (4) training attention, (5) refining awareness, (6) cultivating wisdom, and (7) serving others (Walsh, 2007). These principles suggest that the shaman has developed a method of approaching the world that is unique and also required for the development of his/her discipline. This will be further discussed in what Walker (2008) refers to as the organizing principle of what motivates the shaman to perform his/her work.

In her research, Walker (2008) considers the concept of symmetry as the organizing principle behind the shaman's methodology. This includes rituals, the tools used, songs, and the relationship that the spirit world has with the shaman and his/her community (Walker, 2008). Symmetry is described by Livio (2005) as the balanced and harmonious occurrence of the divergent forces, energies and qualities that make up the individual. Finding similarities in Jung's archetype of the self (Jung, 1960), Livio (2005) notes that this reflects his understanding of symmetry.

Jung's use of the concept of symmetry was sparse, however we find reference in his work entitled Psychology and Alchemy (Jung, 1968). Jung suggests that "symmetry is an answer to the conflict in the dream" (p. 171). As well, Jung (1968) notes that dreams reflect one side of the dream content, or the issue the person is dreaming about, while the conscious mind experiences the other side during wakefulness. Jung maintains that each side of the reflection "must balance the other as its mirror image" (p. 171), with the right side reflecting the world of consciousness and the left representing the unconscious producing a corresponding world in reverse. Thus, the unconscious order becomes the symmetrical counterpart of the conscious mind and its contents. The center, or culmination of the symmetry, is the intersection of unconscious and conscious minds that correspond to each other despite the inversion of the reflective content. Therefore, the result of symmetry allows for the acceptance of the unconscious content and incorporating it into the general understanding of the world (Jung, 1968). 
As well, Walker (2008) considers Jung's definition of the archetype to describe the fundamental original principle of form and order where the individual psyche and cultural beliefs emerge. As noted, Jung (1959a/1980) suggests that the archetype can vary in detail without losing their basic structure (For a more detailed account please see Chapter 2). For example, the archetype of the devil may change form in dream content, but the motif remains the same. Livio (2005) mentions that symmetry is inherent and lies at the intersection of science, art, and perceptual psychology. Symmetry represents a foundation that creates forms and can evolve into paradigms, archetypes, laws and mathematical objects (Livio, 2005). Livio (2005) suggests that symmetry is understood to be bilateral in meaning acting like a type of mirror reflection of either up/down or front/back. A clear example is the bilateral symmetry seen in representations of the human body (Walker, 2008).

Walker (2008) notes that symmetrical relationships in shamanic cultures represent a worldwide phenomenon, signifying an esoteric representation in two-dimensional form. Furthermore, shamanism is modeled on nature with bilateral symmetrical relationships being perceived as opposites: left/right, front/back, sun/moon, night/day, dark/light, male/female, sky/earth. Each pair has the numinous appeal of the archetype and is kept in balance through shamanic ritual. As well, shamanism uses various transformative methods to influence this world of polarity. These include offerings to spirits to ensure balance between this world and the spirit world and animal bones used as tools in the shaman's tool kit as examples of the animal's abilities it had during its lifetime. For example, the shamans of Peru see the eagle's feather as direct connection to the spirit of that animal. The eagle brings forth visions, clarity and foresight with the ability to perceive the world from above, without interfering in it. Thus, the energy of the eagle allows us to gain clarity in our lives (Villoldo, 2000). Therefore, the shaman is human and nature united and embodies the duality of animal and spirit world. By following the myths 
and beliefs of their culture, the cosmology of the shaman's world is preserved (Walker, 2008). Thus, symmetry is maintained and is strengthened each time a ritual is performed.

Therefore, in consideration of the above, similarities in the definition of shamanism exist. The shaman is an individual who has moved through a death and rebirth process. This process involves a psychological transformation whereby the shaman has had a psychological death of his/her old self and a rebirth of a new self, the shaman. This transformational process has established within the shaman an ability to interact with spirits through an understanding of myth and culture, utilizing the ASC to access important information. He/she is described as a charismatic individual using his/her extensive understanding of the ASC to further the overall wellbeing and health of the community (Eliade, 1964/2004; Krippner, 2012; Walsh, 2007; Winkelman, 2010). Shamanism as a religion remains unclear however what is clear is that the shaman possesses an intimate knowledge and understanding of culture, mythology and symbolic meaning (Walsh, 2007; Winkelman, 2010; Krippner, 2012). This allows the shaman to navigate the ASC willingly (Walsh, 2007).

It is also evident that Walker's (2008) notion of symmetry and balance is a key aspect to the definition of the shaman. Winkelman (2010) also brings reference to this idea stating that ritual communication with the spirit world allows for a psychological balance, catharsis and integration to occur. Therefore, the definition of a shaman includes: a charismatic man or women who has passed through a death and rebirth process, utilizing the ASC willingly to help the individual or community attain a psychological, spiritual and physical balance (Ogembo, 2005; Walsh, 2007; Winkelman, 2010). Rooted in culture, the shaman has a deep understanding of cultural, ritual and mythological traditions, drawing upon this at will to help his/her community (Harner, 1980/1990; Levio, 2005; Walker, 2008; Walsh, 2007; Winkelman, 1986; 2010). 
Now that an overview of the research on shamanism has been explained, the thesis will continue its focus on the shaman from the point of view of the archetype of the wounded healer. Even though a general understanding of the wounded healer has become popularized in mainstream culture, seeing both shamanism and the wounded healer representing archetypal configurations from a Jungian perspective is important as its implications imply an archetypal process accessible to everyone who is in the helping profession (Merchant, 2012). As Jung (1961/1990) has emphasized, "only the wounded physician heals" (p. 155).

\section{The Shaman as the Wounded Healer}

A clear understanding of Jung's psychology is outlined in Chapter 1 where his psychology of the psyche, including a description of the archetypes and the archetype of the wounded healer are described. The premise of this section is to discuss the shaman as the wounded healer.

Jungian, cognitive and neuro-phenomenological approaches to archetypal experience offers a view of the human psyche that originate from concepts found in Ancient Greece and throughout European philosophy, religion and art (Scott, 2014). As mentioned previously, Jung's position on the archetype is universal, spanning across culture and belief. Its function is to organize psychic material into patterns that have functional significance despite variances in cultural expression (Jung 1959a/1980). Due to this, Jung (1967/1970) has concluded that the psyche has two primary modalities: (1) logical thinking that is verbally expressed, communicated outwardly, following a train of related ideas expressing direct experiences received from the surroundings; and (2) non-directed thinking or dreaming, an associative process whereby images are more concrete than abstract. The commonality shared amongst these trains of thought reveal that two distinct forms of cognition occur: an ordinary state of consciousness (OSC) and an 
introspective mode of thought comprised of internal imagery to what Jung refers to as the archetypes (Scott, 2014).

Harner (1990) noted that his work with shamans have outlined this structure, calling them ordinary reality and non-ordinary reality. Cognitive-pragmatic approaches also take a similar view noting an inner mental world and the external world of perception (Scott, 2014). Further to this, Noll (1985) concludes that shamanism is a cultural tradition that encourages the cultivation of mental imagery, devoting itself to the induction of this experience, thus supporting the archetypal position of this process. As well, Winkelman's (2010) position on shamanism extends Jung's notions on conscious experience by identifying structures that mediate consciousness in the brain, calling it the Integrative Mode of Consciousness (IMC; for a more detailed account of this please see Winkelman's IMC theory in Chapter 4).

Seeing the ASC as his foundation of the shaman's experience, Winkelman (2010) supports the idea that consciousness must adhere to both the neurobiological and phenomenological experience and reflects the division of the two distinct modes of awareness, paralleling Jung's theory of conscious and unconscious experience (Scott, 2014). Further to this, Kirmayer's (1993) theory of symbolic healing suggests that myth and archetype are essential to ritual healing as they combine biological and experiential meaning together linking the interactions of the body with imagination and society providing a path whereby healing can occur.

Merchant (2012) notes that the commonality reflected in the research on shamanism is the death and rebirth process, the use of the ASC, and the wounded healer archetype. Therefore, this pattern should be recognized across ethnographic studies on shamanism (Merchant, 2012). This has been accomplished in Merchant's analysis of Eliade (1964) and Peters and PriceWilliams (1980), where shamanism is seen to have a core meaning that is expressed through its 
functionality in cultures around the world. This core meaning is reflective in the definable imagery the shaman experiences (Merchant, 2012). To this, Eliade (1964) notes that shamanism is "the very dialectic of the sacred" and indeterminately "repeats a series of archetypes" at specific junctures in time that are "structurally equivalent" to those that occur in the past or the future (p. xvii). For example, world wars are an illustration of a repeating archetype that has occurred in the past and still continues today in different forms, such as cyber warfare.

Merchant (2012) concludes that the notion that such a repetition occurs in shamanism is definable evidence of an archetypal process. Furthermore, the author notes that since Eliade's (1964) research reveals cross-cultural occurrences of shamanism, it reinforces Jung's position that archetypal processes are not static (Merchant, 2012). Since the archetype operates as an organizing principle whereby specific individualized experiences can form, the beliefs of the particular culture therefore influence how the archetype is expressed to the individual. In this case, it provides strong evidence that the shaman is an example of the wounded healer archetype (Merchant, 2012).

Jung concludes that the process of constellating an archetype is representative in an experience that expresses the archetypal process (Merchant, 2012). For example, Crow Dog a Lakota Medicine Man mentions in his book Crow Dog that the sacred pipe is the manifestation of the Great Spirit also know in Lakota as 'Wakan Tanka'. The communication he receives from the Great Spirit is for the healing of the people that come to him. Crow Dog notes:

The pipe lifted up in prayer forms a link between man and Tunkashila. It's a spiritual bridge to the Great Spirit. With the pipe I can communicate with Grandfather Spirit, whom we also call Wankan Tanka, the great sacredness... The pipe is not a thing, it is alive. (p. 133) 
As well, Smith (2007) argues that the shaman's ability to heal arises from the awareness of his wounds and the mastery of tending that wound. Thus, the death and rebirth process synthesizes this change transforming the initiate shaman into the shaman in his/her complete form (Walsh, 2007; Winkelman, 2010). Smith (2007) mentions that this experience is both a psychological and spiritual process representing a transition from a primitive level of consciousness to an evolved level of understanding.

In this classic experience, the initiate shaman experiences a symbolic death of the old self (ego) allowing for a new identity to emerge and the integration of the shaman archetypal pattern to occur (Winkelman, 2010). To this Jung (1969/1980) mentions that,

He (shaman) is essentially unconscious and therefore in need of transformation and enlightenment. For this purpose, his body must be taken apart and dissolved into its constituents, a process known in alchemy as divisio, separatio, and solutio, and in later treatises as discrimination and self-knowledge. (p. 191)

Smith (2007) concludes that the shaman's evolution of consciousness not only adheres to the wounded healer archetype but also is an example of Jung's individuation process. For a detailed understanding of this, please refer to the section on the Self and the Individuation Process outlined in Chapter 1. Briefly, Jung discussed individuation as embracing our own uniqueness, resulting in an inner psychological fulfillment. This coming to "selfhood" (p. 173) is described by Jung (1954) as reaching the wholeness of the human experience, allowing the person to achieve the fulfillment of his/her nature. Once achieved Jung, notes that it relieves the person of the "false wrappings of the persona" (p.174), shedding them from the suggestive influence that others have over them (Jung, 1954).

According to Sandner (1997/2012), Smith (2007) and Merchant (2012), Jung saw the shaman's development as a direct example where the individuation process was projected 
archetypically. The wounded healer archetype is part of this process, where the shaman uses his wounds as a catalyst towards change, both individually and for his public audience (Merchant, 2012; Sandner, 1997/2012).

Reflected through ritual, myth and dress (Winkelman, 2010), the archetype of the shaman is manifested through the unconscious will that brings it to life. Seeing the shaman's wounds as the central agent to healing, Merchant (2012) reflects that Jung saw that the personal experience of the wound allowed the healer or shaman to connect with the wounds of the patient. Unconsciously, the shaman utilized aspects of the wounded healer archetype, fulfilling the archetypal pattern of individuation (Merchant, 2012). Thus, Merchant (2012) notes that the archetypal characteristics of the shaman include (1) mastery, obtained through the death and rebirth experience, (2) dismemberment, (3) the rebirth of a new psyche and, (4) healing rituals performed by shamans for the individuals they are helping. This provides further support that the shaman is a person who has been archetypically influenced (Merchant, 2012).

The conceptual role that shamanism has in analytic psychology is based on the understanding where the conscious experience is acted out in the interplay of the wounded healer and its role in the individuation process (Merchant, 2012). Sandner's (1997/2012) work on analytic psychology and shamanism furthers this notion and suggests that Jung saw the shaman as an archetype, not a unique figure in culture. This not only supports the notion that the archetype is universal, it also removes the allure that shamanism has created in Western culture, suggesting that any human can constellate this archetypal pattern (Jung, 1959a/1980).

Now that an understanding of Carl Jung's work, the shaman and the shaman as a wounded healer has been offered, the ASC will be discussed giving the reader a solid understanding of the research offered in this area of study. The chapter will discuss the 
following: an overview of the research on consciousness, quantum theory and a review of the relevant research on the ASC, and a critical review of the shamanic state of consciousness. 


\section{CHAPTER IV}

\section{THE ALTERED STATE OF CONSCIOUSNESS}

\section{What is Consciousness?}

Over the past 20 years, psychology has renewed its interest in the phenomenology of consciousness with a greater focus on qualitative methods being applied with triangulating thirdperson quantitative methods in psychological research (Velmans, 2007). As well, combined research methods of first- and third-person accounts are used in neuropsychology such as in the search for neural correlates of consciousness using neuroimaging techniques (Rees and Frith, 2007).

In their literature review, Rees \& Frith (2007) suggested that unconscious processing is not based on behavior alone. The authors note that reliable observations in unconscious activation of the human ventral pathway has been outlined by research. For example, Haynes and Rees (2005) showed that conventional fMRI was used to reveal feature-selective processing in the human cortex even when the subjects were masked. As well, Moutoussis \& Zeik (2002) developed a psychophysical technique that dissociated the visual precept from the visual stimulus allowing for brain activity of the perceptual state to be identified distinguishing it from other stages of stimulus processing. Using this technique in the study, the researchers found that when house-specific and face-specific images were used, the specific brain areas associated with this were activated regardless of them being perceived. The authors noted that no "terminal perceptual" (Moutoussis \& Zeik, 2002, p. 9527) area of the visual brain exists, however brain regions involved in processing a visual stimulus are involved in perception. The authors concluded that the difference between the two is the result of a higher level of activity in the specific brain area when the stimulus is perceived (Moutoussis and Zeik, 2002). Further to this, Baumeister et al. (2010) show that conscious thought influences behaviour by activating and 
stimulating motivation, as well as reducing it. Moreover, the authors state that recalling events and reflecting on situations can also influence behavior outcomes. Finally, the researchers imply that if a person has multiple motivations that are competing with one another, consciousness may help choose the best outcome. This suggests that conscious thoughts interacted with existing motivations (Baumeister et al. (2011).

Researchers in the field such as Beauregard (2007; 2014) and Rees and Frith (2007) note that a plethora of evidence now exists concerning how processing outside awareness influences behavior. For example, work demonstrated by Naccache et al. (2005) recorded brain potentials from the amygdala region that is thought to respond to fearful or threatening stimuli. The results showed that subliminal presentation of emotional words modulated the activity of the amygdala, indicating that subliminal words can trigger long-lasting cerebral processes, such as semantic access to emotional change (Naccache et al., 2005). This suggests that an unconscious element may influence how our conscious perception of events, or words on a page, are contrived.

Similarly, unconscious process may also influence cultural behavior. This is due to the variations in the expression of consciousness commonly seen in the expression of dress, art, food, etiquette and behavior. Therefore, cultural expression of the conscious experience is understood to possess differences, with unique expressions altering the perception of how individuals live their lives (Presti, 2011; Whitehead, 2011). Thus, the questions that come to mind include: Are unconscious processes involved in the variations of cultural experiences? Or, how does one culture know how to express itself? However this has occurred, subjective expression has allowed for cultures to influence one another reflecting differences in the expression of consciousness. Chalmers (2007) and Shear (2007) note that scientific methods of deduction fall short of capturing the essence of what the conscious experience is really like, thus being able to explain why cultural variability exists remains challenging. Due to this, research on 
the conscious experience has arrived at two distinct problems: the easy problems of consciousness and the hard problems of consciousness (Chalmers, 2007).

The easy problems of consciousness include explaining how the following phenomena occur: (1) the ability to discriminate, categorize, and react to stimuli from the environment, (2) the integration of information by cognitive means, (3) the reporting of mental criteria, (4) the ability access internal states, (5) the focus of attention, (6) the control of behaviour, and (7) the difference between wakefulness and sleep. Chalmers (2007) mentions that science can explain the easy problems of consciousness by computational and neural mechanisms. For example, to explain the integration of information, science can deduce this by noting the mechanisms involved in bringing information together and then explaining how it is exploited by latter processes. Chalmers (2007) concludes each of the 7 criteria can be explained by an appropriate cognitive or neurophysiological model.

Chalmers (2007) contends that the hard problem of consciousness is explaining the experience of $i t$. Therefore, defining the hard problem using reductionist methods is inadequate because it cannot describe why the experience of consciousness occurs, nor can it explain why performance of these functions are accompanied by experience. Finally, the methodological challenges in measuring consciousness becomes problematic due to its subjective quality (Chalmers, 2007).

Explanations in how to describe the experience of being in consciousness may be reflected in Dennet's (2001) view who argues that consciousness is not relegated to a specific area in the brain or a series of experiences, rather it is an occurrence that exists over time. Taking this into consideration, the experience over time of being in consciousness allows for the knowledge of the experience of consciousness to be formed. Thus, when faced with visual 
stimuli, the experiences of consciousness are recalled, and the expression is the individual's subjective experience.

In support of Dennet (2007), Fischer (1981) concludes that analytic reasoning has a strong influence on our perception noting that induction classifies the familiar and deduction brings it into our understanding. Thus, our experience of consciousness over time allows for this to occur. Fischer (1981) suggests that it is the interaction with consciousness that determines our experience. Hence, we only know a lemon to be bitter once we have tasted it (Fisher, 1981). As Fischer states, "matter evolves to the awareness of its own knowledge and becomes conscious of its self-interpretation" (p. 19). Therefore, the creation of reality may be dependent on the existence of the self-reflective function of our own observation (Fisher, 1981). Finally, Fischer (1981) notes that in a self-reflective process being (ontology) and knowing (epistemology) are united with self-reflection blending both into the conscious act of self-in-observation. Similarly, Wilbur (1977/1993) points out that subject and object are two different ways of approaching consciousness and to forgo this realization sets the opposites against one another, setting up the dichotomy once again. This causes us to believe that an external world exists apart from us, whereas both subject and object are connected allowing for the conscious experience to unfold (Lanza \& Berman, 2009).

\section{Quantum Physics}

Quantum theorists have also arrived at this conclusion. The Copenhagen interpretation, offered by Niels Bohr and Werner Heisenberg, suggests that reality does not exist until it is perceived, and depending on the understanding, multiple complementary aspects of reality will become known (Lanza \& Berman, 2009; Wolf, 2001).

The act of observation allows for the disruption of reality to occur, influencing the results of the perceiver. Therefore the 'true' reality is not unbroken until the observer attempts to create 
it (Lanza \& Berman, 2009; Wolf, 2001). Thus, paradoxes emerge as there is no well-defined line between us and the reality, we believe to be outside of ourselves (Lanza \& Berman, 2009). Examples in psychology include work done with Borderline Personality patients through mentalization, where the patient's perception of reality is challenged (Bateman \& Fonagy, 2006). Bateman and Fonagy (2006) note that beliefs, wishes, feelings and thoughts, internal or external to our awareness, determine what we do. Thus, the use of mentalization is the intentional focus on mental states in oneself, or others, to determine what is really occurring. Recalling work highlighted by Cardeña (2011b), he indicated that the experiences of those who have the diagnosis of borderline personality disorder suggest that perception is altered to suit the individual's model of reality.

According to Einstein's theory of general relativity, matter does not exist independent of space and time. If one is absent, they all are. Space is required for matter to exist; matter is needed for time to exist; and time is required for space to exist. Thus, all are codependent of each other. If time is an experience of the mind, is it real? This puts forward the notion that space and matter may be as well. If observation requires time, then without observation, time would cease to exist (Wolf, 2001).

Wolf (2001) notes that when quantum physicists want to determine the probability of an occurrence, they calculate a number based on the multiplication of two mathematical functions called quantum wave functions. These are speculated to be real waves moving through space and time and cannot be measured, as they have no mass nor energy, and exist solely in our minds.

Further to this, Wolf (1991) notes that subtle vibrations are present and are contained in probability waves that determine how physical events occur. He noticed this while he participated in ayahuasca ceremonies with shamans gaining insight and awareness into the nature of the shaman's experience. In particular, during Wolf's ceremonial experiences with ayahuasca 
with Peruvian shamans, he noted that shamans see the world as vibrations. From his understanding of probability waves, Wolf (1991) determined that it is in the amplitude or strength of these waves that manifest physical events. Thus, this new way of observing how physical events manifest in reality suggests that everything in the universe has this built-in probability-vibrational pattern. Noting the unusual nature of this phenomena, Wolf (1991) suggests that when two particles interact, the probability waves entangle, despite them being miles away from one another. Thus, a connection exists despite them being physically separate from one another. It is in the observation of one particle that creates the effect on the other (Wolf, 1991).

Now that an understanding of how subjective accounts influence our consciousness experience has been outlined, our focus now turns to the relevant research on the altered state of consciousness (ASC).

\section{What is an Altered State of Consciousness?}

As Fisher (1981), Chalmers (2007) Wilbur (1991) and Wolf (1991; 2001) have suggested, our consciousness experience has been influenced by what we perceive reality to be, posing the idea that we inadvertently alter our own perception of reality. In his research on consciousness, Ellis (1995) suggests that conscious information processing involves the use of an image or concept of what might be and is put forward by the individuals' own understanding of it. Thus, the meaning is determined by a subjective view, or if enough people possess the same beliefs, a shared cultural view. Whitehead (2011) furthers this noting that human culture has constructed "profound alterations" (p. 182) in consciousness due to the biological impetus for survival. This "generalized altruism" (p. 182) has allowed for kinship and cooperation, and is held together by rules, obligations, and laws, or an altered form of approaching consciousness 
(Whitehead, 2011). Consequently, our cultural beliefs, cultural norms and values provide evidence that the ASC covers a diverse range of human experience (Mishara \& Schwartz, 2011).

As a result, ethnographic and anthropological research into the nature of the ASC has provided evidence for a cross-cultural basis that extends worldwide (Eliade, 1951/2004; Winkmelan, 2010). Cardeña (2011a) suggests that research has remained distant from the implications that the ASC has on the nature of consciousness and our approaches towards reality. As well, he mentions that research in this area has overlooked that we transition into different states of consciousness regularly, with each displaying different configurations of cognition, emotion, physiology, and behavior. From drug induced states, hypnotic induction, psychopathological states to sleep/wake cycles and daydreaming, research has made accounts of the ASC experience noting its similarities and differences (Cardeña, 2011a).

Cardeña (2011a) notes that many problems exist, such as explaining how we have become conscious and the issues explaining the relationship between qualia (internal/subjective aspects of sense perception due to interaction with phenomena) and neurological processes. Finally, he argues that confusion in consciousness studies centers around the description of the term consciousness with little agreement being made as to the definition of the term (Cardeña, 2011a).

By the end of the 19th century, research lent its focus towards multiple consciousness, dissociation, hypnosis and mediumship (Cardeña, 2011a). For example, psychologist and philosopher William James offered a description on the nature, function and dynamics of consciousness by focusing on dreams, hypnosis, pathological and non-pathological forms of dissociation, genius and parapsychological phenomena (Taylor, 1983). In his reflections on the conscious experience, James (1910) notes that "the best way in which we can facilitate their advent is to understand how great is the darkness in which we grope, and never forget that the 
natural-science assumptions with which we started are provisional and revisable things." (p. 468)

Despite advances like these occurring in the study of ASC during the 18th and 19th century, the arrival of psychoanalysis and behaviourism within academic psychology diminished the study of ASC to obscurity until a few decades ago (Cardeña, 2011a). Thus, research in the ASC is still in its infancy with extensive work on the ASC not occurring until the mid 1950's (Cardeña, 2011a).

\section{Recent Contributions}

Ludwig's (1966) review of the literature on the ASC sought to accomplish (1) the circumstances needed for the occurrence of the ASC; (2) the factors that allow the ASC to manifest; (3) commonalities amongst the different theories on ASC; and (4) the adaptive or maladaptive functions that these states have for people. Ludwig's (1966) conclusions defined an ASC as a mental state that is subjectively recognized by the individual or observer. Representing a significant deviation of psychological functioning from the normal waking consciousness, the ASC can be induced by various physiological, psychological, or pharmacological manoeuvres or agents (Ludwig, 1966). For example, after an extended period of dancing, drumming and singing, shamans are known to collapse into a dreamlike state because of extreme exertion on the physical body (Gackenbach \& LaBerge, 1988).

Ludwig (1966) identified ten characteristics of an ASC: (1) alterations in thinking; (2) disturbed time sense; (3) loss of control; (4) change in emotional expression; (5) body image change; (6) perceptual distortions; (7) change in meaning or significance; (8) sense of ineffable; (9) feelings of rejuvenation; and (10) hyper-suggestibility (ability to automatically respond to demands, increased tendency to misinterpret outward stimuli based on emotional experience). As well, Ludwig (1966) mentions that an ASC can occur in any setting through a variety of means 
that interfere with the ability to sense emotions, outward stimuli, the regulation of motor impulses and the organization of cognitive processes. Please see Table 1 and Table 2 for an account of Ludwig's (1966) findings. 
Table 1

Ludwig's General Characteristics of the ASC

\section{ALTERATIONS IN THINKING}

- Subjective disturbances in concentration, attention, memory, and judgement

- Archaic modes of thought (primary process thought)

- Predominate, and reality testing is impaired to varying degrees

- Distinction between cause and effect becomes blurred, ambivalence increases, and incongruities or opposites can coexist without any psychological conflict

\section{DISTURBED TIME SENSE}

- Sense of time and chronology became greatly altered

- Subjective feelings of timelessness, time coming to a halt, acceleration of slowing of time

- Time may seem infinite or infinitesimal in duration

\section{LOSS OF CONTROL}

- Upon entering the ASC, experience of fear, losing grip on reality, self-control occurs

- During induction phase (sleep, hypnosis, anesthesia) resistance to the onset of ASC occurs

- In other instances, it is welcomed such as in narcotic drug use (alcohol, LSD, mystical states)

- Feelings of impotency, feelings of gaining increase control or power occur despite a sense of loss of control (occurs with hypnotized persons, audiences who identify with the power of omnipotence acquired from the hypnotist)

- Relinquishing of control during mystical, revelatory, or spirit possession states in hopes to experience divine truth, clairvoyance, "cosmic consciousness", communication with spirits/supernatural power, serving as a temporary mouthpiece to the gods

\section{CHANGE IN EMOTIONAL EXPRESSION}

- Sudden, unexpected displays of intense emotion

- Emotional extremes (from ecstasy to profound fear and depression)

- Detachment, relation of intense feelings without any emotional display

- Capacity for humour may diminish

\section{BODY IMAGE CHANGE}

- Wide array of distortions in body image occur

- Profound sense of depersonalization, a schism between body and mind

- Feelings of derealization, dissolution of boundaries between self and others, world and universe

- Parts of body feel shrunken, enlarged, distorted, heavy, weightless, disconnected

- Spontaneous experiences of dizziness, blurring of vision, weakness, numbness, tingling, and analgesia

Note. Interpreted from Ludwig, C. (1966, p. 227-230). The text was used to create Table 1. 
Table 2

Ludwig's General Characteristics of the ASC

\section{PERCEPTUAL DISTORTIONS}

- Hallucinations, pseudo-hallucinations, increased visual imagery, subjectively felt hyper acuteness of perception, illusions of every variety

- Content of hallucination may be determined by cultural, group, individual, or neurophysiological factors representing wish-fulfillment, expression of basic fears or conflicts/phenomena of little dynamic import (hallucinations of light, color, geometrical patterns, or shapes.

- ASC's produced by psychedelic drugs, marijuana, or mystical contemplation create experiences whereby one form is translated into another form such as feelings sounds or tasting what is seen

CHANGE IN MEMORY OR SIGNIFICANCE

- Increased meaning or significance to subjective experiences, such as having a "eureka" experience

- Feelings of profound insight, illumination, truth frequently occur

- During toxic or psychotic states, increase in false significance to external cues, ideas of reference, numerous instances of "psychotic insight"

\section{SENSE OF INEFFABLE}

- Ineptness or inability to communicate nature or essence of the experience

- Development of amnesia during hypnotic trances, somnambulistic trance, possession fits, dreaming, mystical experiences, delirious states, drug intoxication, auras, orgiastic and ecstatic states

\section{FEELINGS OF REJUVINATION}

- Upon emerging from profound ASC stats such as after psychedelic use, a new sense of hope, rejuvenation, renaissance, or rebirth is experienced

\section{HYPERSUGGESTIBILITY}

- Increased susceptibility of persons to accept and/or automatically to respond to specific statements (commands or instructions of a leader, shaman, demagogue or hypnotist)

- Increased tendency to misperceive or misinterpret various stimuli or situations based either on inner fears or wishes

Note. Interpreted from Ludwig, C. (1966, pp. 227-230). The text was used to create Table 2. 
Tart (1975) offered more to Ludwig's (1966) definition by describing an ASC as qualitatively different from a baseline state, identifying it as an anomalous experience. In his work with deep hypnosis and his systematic review of the literature, Tart (1975) offered taxonomical and phenomenological contributions, suggesting that consciousness is a predetermined existence based on our individual, societal and cultural experiences, and that we take reality for granted, considering it normal or ordinary consciousness. Tart (1986) notes that a stabilized state of consciousness is one that maintains its overall pattern despite fluctuating changes in the outward environment. It is only once this stabilization changes, such as being induced into an altered state, that "the nature of conscious experience can change drastically" (Tart, 1986, p. 5). In his conceptualization, Tart (1975) described states of consciousness as conditions that are qualitatively different from others by the existence of conditions that are absent in other states. Linked to the concept of ordinary consciousness, Tart's paradigm for altered consciousness is conceptualized as states of consciousness (SoC), discrete states of consciousness (d-SoC), baseline states of consciousness (b-SoC), and a discrete altered state of consciousness (d-ASC; Tart, 1977). A discrete altered state of consciousness is a new system of consciousness with unique properties, constituting a restructuring of consciousness (Tart, 1975). The criteria are listed in Tables 3 and 4 on p. 77, 78.

In his preview to Altering Consciousness, Tart (2011) emphasizes that a key distinction between the various subjective experiences in consciousness and the ASC is that "the ASC must be assessed by reported or observed significant changes in the quality of a person's consciousness" (Tart, 2011, p. xiii). Tart reflected that his systems approach to the ASC "allowed for emergent properties as various subsystems changed their functioning and a general analysis for inducing and maintaining altered states" (Tart, 2011, p. xvii-xix). According to Tart (1975) ten major subsystems identify key variations of the d-ASC. They are listed below in Table 3 and 
Table 4. Table 3 is broken down into the following: (1) Exteroception (being aware of the external environment; (2) Introception (being aware of what the body is feeling and doing); (3) Input-Processing (an automated process that determines what is important by a person to be relevant and is based on cultural standards; (4) Emotions; and (5) Memory. Table 4 concerns the following criteria: (1) Space/Time Sense (our ability to construct psychological space and time); (2) Sense of Identity (the subjective perception of reality that makes it a personal experience); (3) Evaluation and Cognitive Processing; (4) Motor Output (muscular and glandular outputs to the external world and the body); and (5) Interaction with the environment. 
Table 3

Experimental Criteria for Detecting an Altered State of Consciousness

EXTEROCEPTION (sensing the external world)

- Altering in various sensory characteristics of the perceived world--glowing lights at the edge of things, attenuation or accentuation of visual depth

INTROCEPTION (sensing the body)

- Altering in perceived body image--shape or size

- Alteration in detectable physiological parameters--accelerated or retarded heart rate, respiration rate, muscle tonus, tremor

- Perception of special bodily feelings not normally present--feelings of energy in the body, generally or specially localized, as in the spine; change in quality of energy flow in the body, such as intensity, focus vs. diffuseness

INPUT-PROCESSING (seeing meaningful stimuli)

- Sensory excitement, involvement, sensuality

- Enhanced or decreased sensory intensity

- Alterations of dominance-interaction hierarchies of various sensory modalities

- Illusion, hallucination, perception of patterns and things otherwise known to be unlikely actually exist in the environment

\section{EMOTIONS}

- Alterations in emotional response to stimuli--overreacting, not reacting, reacting in an entirely different way

- Extreme intensity of emotions

MEMORY

- Changes in continuity of memory over time-either an implicit feeling that continuity is present or an explicit checking of memory that shows current experience to be consistent with continuous memories up to the present, with gaps suggesting an altered state

- Details. Checking fine details of perceived environment (external or internal) against memories of how they should be to detect incongruities.

Note. Table 3 has been adapted into two tables, Table 3 and Table 4 from Tart, C. (1975, p. 12,

Table 2.1) entitled Experimental Criteria for Detecting an Altered State of

Consciousness. Permission was granted by Tart to reprint the tables. Please see Appendix A. 
Table 4

Experiential Criteria for Detecting an Altered State of Consciousness

TIME SENSE

- Unusual feeling of here-and-nowness

- Feelings of great slowing or speeding of time

- Feeling of orientation to past and/or future, regardless of relation to present

- Feeling of archetypal quality to time; atemporal experience

\section{SENSE OF IDENTITY}

- Sense of unusual identity, role

- Alienation, detachment perspective on usual identity or identities

EVALUATION AND COGNITIVE PROCESSING

- Alteration in rate of thought

- Alteration in quality of thought - sharpness, clarity

- Alteration of rules of logic (compared with memory of usual rules)

MOTOR OUTPUT

- Alteration in amount or quality of self-control

- Change in active body image, the way the body feels in motion, the proprioceptive feedback signals that guide actions

INTERACTION WITH THE ENVIRONMENT*

- Performance of unusual or impossible behaviors - incongruity of consequences resulting from behavioral outputs, either immediate or long term

- Change in the anticipation of consequences of specific behaviors - either pre-behavioral or learned from observation of consequence

- Change in feeling of degree of orientation to or contact with immediate environment

- Change in involvement with vs. detachment from environment

- Change in communication with others - incongruities or altered patterns, consensual validation or lack of it

* This category represents the combined functioning of several subsystems

Note. Table 4 has been adapted into two tables, Table 3 and Table 4 from Tart, C. (1975, p. 12,

Table 2.1) entitled Experimental Criteria for Detecting an Altered State of

Consciousness. Permission was granted to reprint the table by Tart. Please see Appendix A for details. 
Similarities in the observations of Ludwig (1966) and Tart (1975) include both researchers acknowledging the following as indicators of an ASC: (1) alterations in thinking; (2) a disturbed sense of time; (3) a loss of control of the environment; (4) noticeable change in emotions; (5) body image distortions; (6) perceptual changes through visual, subjective hallucination; (6) memory change; (7) hyper suggestibility and; (8) a sense of ineffability. What was not identified explicitly by Tart (1975) was the feeling of rejuvenation and a sense of rebirth or renewal that Ludwig (1966) notes occurs after psychedelic drug use.

As research progressed, discoveries on ASC states and phenomenal aspects began to emerge (Cardeña, 2011a). For example, Fischer's (1986) research established a continuum of states of consciousness that focused on sympathetic/parasympathetic intensity. These include: (1) ecstatic, (2) hyper-aroused, (3) aroused, (4) normal, (5) tranquil, and (6) hypo-aroused (Fisher, 1986). In turn, each state has a descriptive level(s) accompanying each state (Fisher, 1986). For example, ecstatic involves a mystical/rapture element and aroused involves anxiety, creativity and sensitivity (Fischer, 1986).

Fischer's systematic review of Western and Eastern approaches to consciousness (1986) suggests that the human experience is defined by a series of subjective and objective experiences that are based on prior individual, cultural, and social experiences. Being human, as Fischer (1986) explains, is both a subjective (being the actor) and a reflective (being the spectator) experience. Similarly, Wilbur (1991) and Whitehead (2011) both suggest that our conscious experience is altered subjectively to support our interpretation of life, suggesting both reflective and subjective qualities. Mentioned earlier on p. 60, these experiences are based upon "recursive reflections" (Fischer, 1986, p. 10) of the self upon a world that reflects an experience that is entirely self-created. Considering the experience of the ASC, Fischer (1986) notes that each state is defined either by a hypo-arousal (deep meditative states) or hyper-arousal (heightened 
awareness), occurring during nervous system excitation. Please see Figure 3 below for a detailed understanding of Fisher's cartography of meditative and ecstatic states. Fisher mentions that the diagram be interpreted as "an immense experiential theatre with revolving stage sets" (Fisher, 1992, p. 8). The inner space of each segment or stage set links to a hyper-aroused and hypoaroused state of consciousness, each focused on a particular innate understanding that is being revealed. This innate understanding has been formed by the experiences of life, layered and reprinted over time (Fisher, 1992). As Fisher mentions, "The stories woven from these themes are constantly re-written, re-painted, and re-composed for each generation with by slight variation in style begun by others" (Fisher, 1992, p. 8).

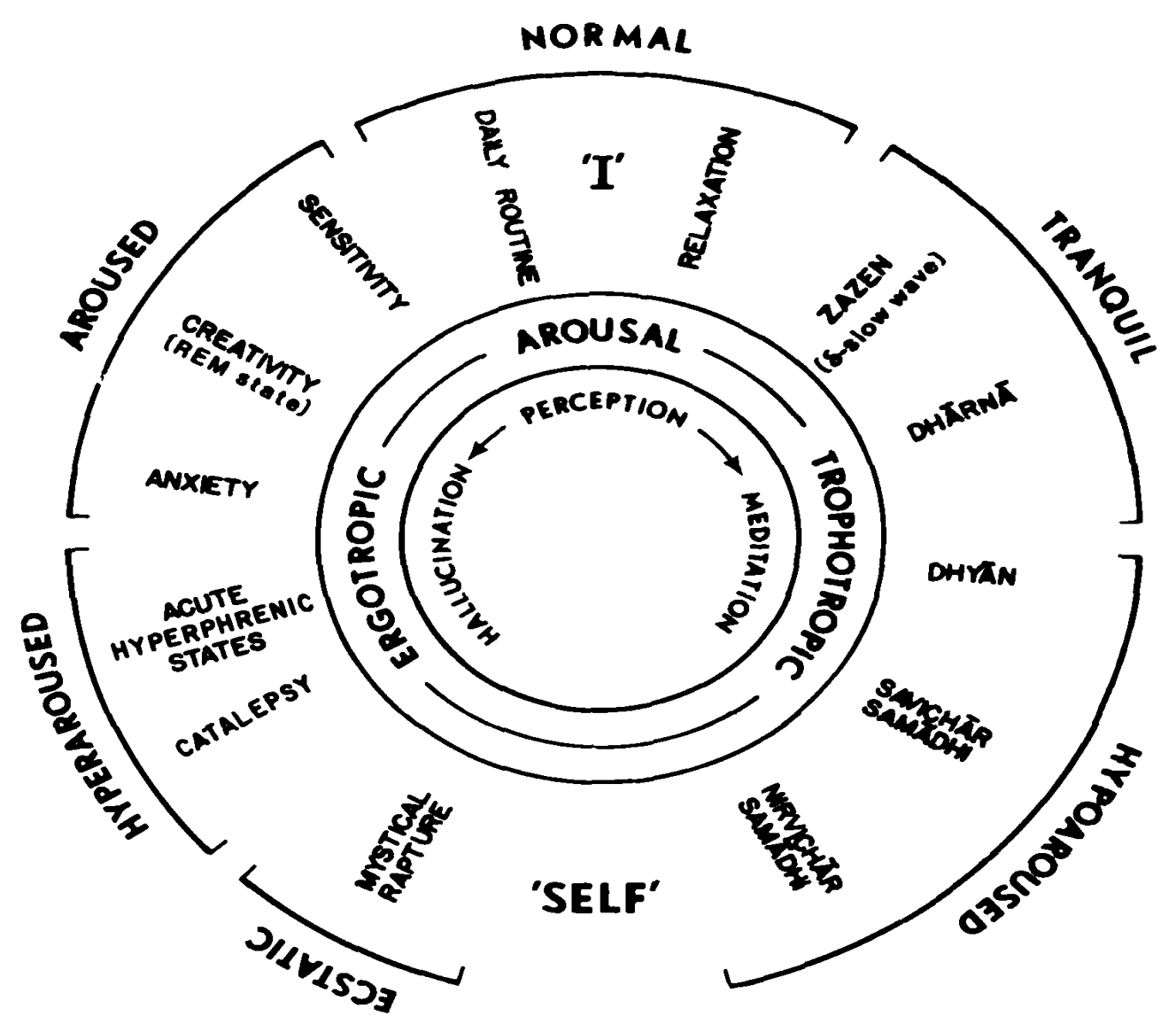


Figure 3. Fisher's: A cartography of states of consciousness (Fisher, 1992, p. 7). It is outlined on a continuum of increased central (ergotropic) or hyper-arousal states on the left side, and perception-meditation continuum on increasing central, or hypo-arousal on the right side. The upper $1 / 3$ of the diagram depicts cognitive states of consciousness, and the lower $2 / 3$ reflects states of consciousness that do not rely on cognition. Fisher (1992) notes that these states are accessible by anyone. Reproduced by permission of the American Anthropological Association from Anthropology of Consciousness, Volume 3, Issue 3-4, pp. 3-13, 1992. Not for sale or further reproduction. Please see Appendix A for email verification.

\section{Induction Procedures}

The induction procedure acts as a bridge allowing a person to enter into the ASC (Presti, 2011). Showing noticeable changes in the subjective and neurophysiological experience where distinguishing states emerge, psychological induction methods such as meditation and hypnosis have provided research with important information on the ASC experience (Cardeña, Steele, 2011a; Winkelman, 2011a). For example, Cahn and Polich (2006) note that in meditation studies research has revealed that brain waves show increased alpha waves (electrical rhythm in brain with a frequency of 8 to 13 hertz) that eventually decrease into theta waves, a high brain wave pattern with a frequency between 4 and 9 hertz occurring in various regions in the brain, such as the hippocampus and cortex (merriam-webster.com). As well, a large amount of research connects ASC induced by psychological induction procedures to changes in brain activity. This includes increased theta EEG activity in the anterior-frontal and frontal-midline regions of the brain producing blissful experiences in subjects (Vaitl et al., 2005). Finally, pharmacologically induced ASC are achieved through psychoactive compounds such as caffeine, alcohol, nicotine and plant-based drugs such as marijuana, cocaine and peyote (Presti, 2011). Interacting with the central nervous system, psychoactive drugs affect the mental processes (perception, mental 
images, thoughts, emotions, sense of self) of the brain altering perception and consciousness (Presti, 2011).

In their research, Vaitl et al. (2005) offered categorical distinctions of the various induction procedures. These include: (1) spontaneously occurring (states of drowsiness, daydreaming), (2) physically and physiologically induced (extreme environmental conditions, as seen in starvation), (3) psychologically induced (sensory deprivation, relaxation, and hypnosis), and (4) disease induced (as seen in psychotic disorders, coma and epilepsy; Vaitl et al., 2005). Aghajanian and Marek’s (1999) literature review suggests that the hallucinogens LSD and mescaline produce a "ubiquitous effect" on cognition, perception and mood, acting as a partial agonist (producing partial effects rather than a full enhancement) to the neurotransmitter serotonin. As well, reviews on methylenedioxymethamphetamine (MDMA) by Parrott (2001) revealed significant serotonin re-uptake distribution in the presynapse (where neurotransmitters bind with receptors such as MDMA). In support of Vaitl et al. (2005), Dietrich (2003) concludes in his review of psychological and neurological indicators of ASC that commonalities in brain functions of ASC are seen in various induction methods such as endurance running, dreaming, hypnosis, drug induced states and meditation.

According to Cardeña (2011a), research on ASC (2001a) often combines qualitatively distinct states of consciousness with induction procedures. Cardeña (2011a) criticizes the work of Vaitl et al. (2005) noting that the researchers do not distinguish between the subjective aspect of altering consciousness (impact of physical environment has on perception of individual) and psychological procedures that may affect the states of consciousness of the participants (Cardeña, 2011a). These psychological procedures include: (1) sensory deprivation, (2) rhythminduced trance by drumming and dancing, (3) relaxation, (4) meditation, (5) hypnosis, and (6) biofeedback (Cardeña, 2011a). Furthermore, Cardeña (2011a) mentions that alterations in 
consciousness created by a hypnotic procedure can also be triggered by other factors such as trauma (Cardeña, 2011a). Further to this, Mishara and Schwartz (2011) note that methodological issues occur in research focusing on healing practices that deliberately induce ASC. The authors argue that phenomenological research on consciousness allows for the creation of a subjective reality, distorting the results (Mishara \& Schwartz, 2011). Since little is known about the mechanisms that are used by healers across the world to deliberately induce ASC in themselves and their patients, a model is needed to understand the therapeutic effectiveness that outlines how induction procedures engage similar or overlapping neural mechanisms or networks in the brain (Mishara \& Schwartz, 2011).

Furthermore, Cardeña (2011a) argues that classifying ASC into an arranged set of biological descriptions, as seen in Winkelman's (2010) IMC that uses Maclean's triune brain theory of synchronization and Fisher's (1986) continuum model are limited because more is involved in the process of ASC than neurological indicators of prior experience (Cardeña, 2011a). Explained on p. 100, Winkelman (2010) argues that the ASC occurs as a result of the synchronization of the reptilian, mammalian and paleo-mammalian brains. Furthermore, Cardeña (2011a) points out that if consciousness can vary, how much can it vary and remain recognizable? Finally, what creates enough of a change to the baseline state of consciousness to warrant an ASC occurring (Cardeña, 2011a)?

Even though Winkelman (2010) argues that an ASC requires the synchronization of the paleo-mammalian, mammalian and reptilian brains, research by Noirhomme and Laureys (2011) suggest that most synchronistic brain (see note 2) states are only found in seizures and comas. The authors note that epileptic seizures are defined by transient signs or symptoms caused by unusual amounts of synchronistic brain activity that affects sensory, motor, automatic function, consciousness, emotional states, memory, cognition and behaviour (Noirhomme \& Laureys, 
2011). Due to the differing opinions of classification and terminology in the current research, Cardeña (2011a) proposes that a classification of an ASC should be clear, offering distinctions to the complex nature of both mental (waking state) and brain events (dreaming). Furthermore, suggestions made by Revonsuo, Kallio and Sikka (2009) on the role that consciousness plays in ASC research also warrants further discussion because until the issues of explaining the cognitive and behavioural functions of the conscious experience (Chalmers, 2007) is achieved, the ability to define the ASC experience will remain to be problematic.

\section{Types of ASC}

Throughout the literature various types of ASC have become well known to research. For the purposes of this review the following will be outlined: the mystical experience (Stace, 1972; Wulf, 2000; Sluhosky, 2011) the transcendental experiences (Levine \& Steele, 2005), Beauregard's (2011) grouping of the RSME (religious/spiritual/mystical experiences) and the psychopathological experience (Cardeńa, 2011b). What follows is a description of each type of ASC with historical and descriptive characteristics.

\section{Mystical Experience}

Early accounts of defining the mystical experience have helped shape the phenomenal criteria of the experience (Sluhovsky, 2011). For example, St. Augustine's description of the mystical experience identified three subtypes: (1) a spiritual subtype consisting of imaginary, hearing/seeing things with spiritual sense (awareness); (2) a corporeal subtype described as an experience perceived through the body, and (3) an intellectual understanding during which the mystic acquires an inward presence independent of any sensory form (Sluhovsky, 2011). However, it was not until the early 1900's when scientific inquiry into the mystical experience began (Sluhovsky, 2011). As mentioned earlier, Harvard psychologist and philosopher William 
James (1905/2008) conducted a thorough review on the nature of the religious experience from an experiential perspective.

In his review of monks whose focus was to experience the divine, James (1905/2008) offered four defining features: (1) ineffability: a mind state that is directly experienced, having no logical explanation involving states of feeling rather than intellect; (2) noetic: involving an experience of knowledge, insight and truth unattainable by the intellect; (3) transiency: there is a limit in the duration suggesting that two hours is the maximum; and (4) passivity: an understanding that another consciousness is present causing the will of the experiencer to cease control and for phenomena such as prophetic speech, automatic writing, or mediumistic trances to occur. However, after the experience ceases, memory of the occurrence may remain, but specific details will not (James, 1905/2008). In a later work entitled Essays in Psychical Research, James (1842/1986) clarifies that during mystical states the consciousness of the person splits into two parts, operating unknowingly and simultaneously together.

Walter T. Stace (1972) provided research with a philosophical, epistemological and a metaphysical account on mysticism. In his reflections on the mystical state, Stace (1960) observed that two types of mystical experiences exist: (1) extrovertive (acquiring the mystical experience through external sources such as nature, art or music), and (2) introvertive (acquiring mystical experiences through an internal identification within). Finally, Stace (1972) concludes that the mystical experience "involves the apprehension of an ultimate non-sensuous unity in all things; a oneness or a One to which neither the senses nor the reason can penetrate" (p. 14).

However, an understanding of the mystical experience was not offered until Underhill's (1930) detailed phenomenological description of the mystical state emerged. Her influences included her spiritual director, Baron Friedrich von Hügel, while also having various correspondences with Indian mystic and author Rabindranath Tagore throughout her life 
(Cropper, 2002). According to Underhill (1930), the defining characteristic of the mystical state is the desire to seek out God. Noting that the mystical state also involves a transitory illuminative state (a deep sense of divine presence) and the intuitive contact with God, Underhill (1930) contends that once the student begins the pursuit towards realizing God nothing else is more important. Cropper (2002) notes that it was Underhill's love of the mystics that inspired her to enter a life of self-study where her love of God was her focus. Self-trained and having no ecclesiastic nor academic training, she gave retreats and served as a spiritual guide to everyday people in search of God. Her dedication to "human holiness" (pg. x) gave her writing a focus and passion that brought her attention from notable writers such as T.S. Elliot who noted that her brilliance stemmed from her understanding of her colleagues' need to incorporate a contemplative aspect to their lives (Cropper, 2002). Underhill (1930) notes that "true mysticism is active and practical” (p. 55), an organic process, involving the entire self, aiming for transcendental and spiritual fulfillment. Furthermore Pargament (2007) acknowledges Underhill (1930) noting that a mystical experience involves feelings of unity, a sense of oneness, a merging with objects, or an experience of unity with pure consciousness characterized as "devoid of discrete perceptual objects (Pargamont, 2007, p. 71).

Wulf (2000) concluded that a key characteristic of the mystical experience is that it must be perceived as real (Nichols \& Chemel, 2011). In his research on the mystical state across religions, Stace (1960) identified seven defining characteristics: (a) the disappearance of all the physical and mental objects with the emergence of a unitary or pure consciousness; (b) the experience of being located in neither space nor time; (c) the sense that objectivity or reality exists; (d) the feelings of peace, joy, bliss; (e) the feeling of experiencing the holy, sacred or divine; (f) the paradoxical feeling of feeling no reality and reality at the same time; and (g) the experience of ineffability. Thus, according to James (1905/2008), Underhill (1930), Stace (1960; 
1972), Pargamont (2007), and Sluhovsky (2011) mystical experiences occur as a result of a (1) unity existing between the person and the outward environment, (2) feeling of peace and joy, (3) paradoxical understanding of there being no reality and reality at the same time, (4) realization that another consciousness is present, and (4) innate understanding of seeing things from an inner awareness without knowledge of how.

In Wulf's (2000) review, he refers to studies conducted in the United States and Great Britain on the prevalence rates of mystical states in the general population. He concludes that even though a sizeable minority of the sample size of 1460 people in the United States (30-40\%) and a sample size of 1865 polled in Great Britain (30\%) report having a mystical experience (Greeley, 1975), actual prevalence of a mystical experience may be lower (Wulf, 2000). The original study by Greeley (1975, p. 58) asked the question "Have you ever felt as though you were very close to a powerful, spiritual force that seemed to lift you out of yourself?" was found to be misinterpreted after replication done by Hufford (1985) revealed a misunderstanding of the question. Hufford (1985) found that 8 out of 14 who responded resulted in a false positive after closely analyzed, as 6 of the respondents understood "lift out" to be metaphorical, and understood being "close to a powerful, spiritual force" with a belief that God was always close by.

Recent studies on the mystical experience have helped shed light on where it occurs in the brain. As mentioned earlier, Beauregard and Paquette (2006) provided research on identifying the neural correlates of a mystical experience in a group of contemplative Carmelite nuns by using fMRI (functional magnetic resonance imaging) to determine where the mystical state occurs in the brain. The authors concluded that several key brain regions and systems are likely to contribute to the mystical experience (Beauregard \& Paquette, 2006). These include: the right medial orbitofrontal cortex in the neomammalian brain, right middle temporal cortex that is 
situated in the reptilian brain region, right inferior and superior parietal lobules, located in the neomammalian brain region, right caudate, situated in the paloemammalian brain, left medial prefrontal cortex of the neomammalian brain, left anterior cingulate cortex in the neomammalian brain, left inferior parietal lobule of the neomammalian brain, left insula in the neomammalian brain, left caudate of the paleomammalian brain, and left brainstem in the reptilian brain. Reflecting on Winkelman (2010), all the above brain regions outlined by Beauregard and Paquette (2006) suggest that mystical states require the synchronization of the paleomammalian, neomammalian and reptilian brain regions to occur.

Other loci of activation were also seen in the extra-striate visual cortex, the area responsible for processing visual information (Beauregard \& Paquette, 2006). This study supports conclusions made by Wulf (2000) who noted that research has identified activity occurring in the temporal lobes during mystical experiences. Noting further that studies on epilepsy have also indicated neural origins of mystical experience, Wulf (2000, p. 405) concludes that the "common and spontaneous, mild forms" of mystical states are difficult to replicate in laboratory settings. This is supported by Halgren et al. (1978) who published findings of electrical stimulation applied bilaterally to the medial-temporal-lobe (MTL) on 36 people. The researchers noted that $92 \%$ failed to induce mental responses (stimulation, images, thoughts, emotional reactions etc.) and that mental alterations evoked by electrical stimulation of the MTL was "variable and idiosyncratic" (Halgren et al., 1978, p. 110).

Despite this claim recent studies by Newberg et. al (2001) and Beauregard and Paquette (2008) reveal the contrary. Newberg et. al (2001) measured changes in regional cerebral blood flow (rCBF) using SPECT imaging to correlate the cerebral activity in eight Tibetan meditators. This allowed for a comparison to be made between $\mathrm{rCBF}$ at baseline and during meditation. The participants used a form of meditation where they focused their attention on a visualized image. 
The 'peak' experience of the meditation is described as a sense of absorption into the visualized image producing clarity of thought and a loss of the usual sense of time and space. Newberg et. al (2001) noted that a significant increase in rCBF occurred in the cingular gyrus, inferior and orbital frontal cortex, dorsolateral prefrontal cortex and thalamus brain regions. Similarly, Beauregard and Paquette (2008) conducted a follow-up study with 14 Carmelite nuns on EEG identifying the neurocorrelates of mystical experiences. The nuns were asked to recall and relive the most intense mystical experience they have felt as a member of the Carmelite Order. The researchers showed marked changes in EEG spectral power and coherence were achieved (Beauregard, 2008).

\section{Transcendental Experience}

Often leading to a deep transformative experience, the transcendental experience (TE) is accompanied by an awareness that reality exists beyond physical and psychosocial awareness (Levine \& Steele, 2005). Researcher's Levine and Steele (2005) conclude that a perception of a merging with "the source of being" (p. 1) is experienced in those individuals who experience a TE. Calling the TE a type of mystical experience, Beauregard (2011a) notes that during the past decade, interest in brain mechanisms associated with TE has become a popular phenomenon in research. This is mainly due to the transformative nature they have resulting in noticeable changes in attitude, behavior, worldview, belief system, relationships and sense of self (Beauregard, 2011a).

Being induced by hypnosis, meditation, epilepsy, near death experiences (NDE) or psychoactive drugs (Cardeña, 2005), TEs create an overwhelming sense of peace, and bliss within (Beauregard, 2011a). Basing his findings on brain research that highlights the limbic system as a key area where TEs may occur, Beauregard (2011a) concludes that describing the feelings of the TE experience is difficult to replicate with words. In his philosophical analysis of 
the literature, Roy (1942/2001) offers a phenomenological account of the transcendental experience suggesting six defining characteristics of a TE: (1) preparation: occurring over a period of days to years this existential occurrence contains aspects of uneasiness, tension and/or struggle (2) immediate occasion: what propels the TE into motion and can be triggered by anything external such as another person an event or a piece of art, (3) feeling of the experience: a feeling that suggests the realization of another reality, (4) discovery: the awareness and understanding that the feeling has happened, (5) interpretation: reflection on the experience involving the awareness and insight into what has happened, and (6) fruit or essence of the experience: the benefit gained such as wisdom, change in attitude and motivation.

Levin and Steele's (2005) comprehensive literature review revealed that two subtypes of transcendent experiences exist: (1) the green type and (2) the mature type. Suggesting that a developmental range exists between the two, the green type involves a transitory nature occurring suddenly or as an outcome to a specific physical or spiritual practice creating a “profound experience of pleasure" (Levin \& Steele, 2005, p. 89). In contrast, the mature type allows for a shift in consciousness into a "new sate of awareness" (Levin \& Steele, 2005, p. 89) thus having a longer effect constituting a tranquil and serene state. Equating the mature type with the mystical state, the authors see the TE as part of the mystical process, concluding that both types of transcendental experiences are created due to a sacred union and through a sense of transcendence (Levin \& Steele, 2005).

Further, the same authors note that within Western traditions, the TE is described as a union with God or the Divine, while within Eastern traditions, the TE is viewed as an identification with all living things. They add that for non-religious individuals, the TE may be experienced as unity with the universe or all of life (Levin \& Steele, 2005). Thus, like the mystical state, a sense of uniting with a source or eternal being also occurs in TE (Levin \& Steel, 
2005). Mentioning that the principal characteristic of the TE is the dissolution of the ego with an awareness that extends beyond the physical body and its psychosocial boundaries, Levin and Steel (2005) suggest that mystics and scholars have attributed an ineffable quality to the TE, concluding that the TE may be the ultimate subjective experience. Therefore, the difficulty that research has is accurately defining it.

In his review of transcendental experiences and the temporal lobe, Beauregard (2011a) concludes that even though clinical studies of epilepsy report the involvement of the temporal lobe and the limbic system, the relationship between them is poorly understood because those who have experienced TE's do not have epilepsy with very few people with epilepsy reporting them. Furthermore, recent studies note that higher mental functions across the cerebral structures of the brain suggest that a larger scale of brain networks that involve the visual and parietal areas of the brain (where the integration of sensory information occurs) are likely responsible for TE's (Varela, Lachaux, Rodriguez, \& Martinerie, 2001, as cited in Beauregard, 2011a).

\section{Religious/Spiritual/Mystical Experience}

In the Handbook of the psychology of religion and spirituality, Paloutzain and Park (2005) conclude that a major theme within the psychology of religion is whether religion and spirituality can be differentiated. They note that researchers such as Hill et al. (2000) share an understanding that scientific definitions of both spiritualty and religion include "a sense of, a belief in, or a search for the transcendent” (Paloutzain \& Parke, 2005, p, 348). Connolly and Light (2003) suggest that some researchers do not distinguish between religious and spiritual experiences keeping it as one term (Koenig, 2000; Barnes, Plotnikoff, Fox, and Pendleton, 2000) while others see spirituality as an umbrella concept, and religion as a manifestation of spirituality (Cohen et al., 2001). Thus, for the purposes of this review the combined term of 
Religious/Spiritual/Mystical Experience (RSME) offered by Beauregard will be used (Beauregard, 2011b).

Beauregard mentions that religious experiences (RE) occur from a religious tradition involving contact with the divine or a religious figure, whereas SEs are largely subjective and do not relate to a religious figure but can bring the person into contact with the divine (Beauregard, 2011b). Similarly, Pargament (1999) states that the term religion is most often identified with a formal or rigid structure, whereas spirituality is described as an individual experience most often identified with personal transcendence and meaningfulness (Spilka \& McIntosh, 1996). Beauregard (2011b) points out that despite MEs not being characteristically religious, they are comprised of the foundation of most religious traditions. The famous mystics of Christianity for example, such as St John of the Cross and Teresa de Avila, are known for their pursuit of God (Underhill, 1930). Mysticism, according to Beauregard centers on establishing communication or an identification with the divine through a direct and immediate experience (Beauregard, 2011b). Noting that RSME's are brain mediated, Beauregard (2011b) concludes that fundamental life changing aspects can occur, such as a state of cosmic consciousness, defined as a union with the divine, visions, and near-death experiences (Beauregard, 2011b).

Anthropologist Charles Whitehead whose research focuses on bridging the gap between social and biological anthropology, notes that both SE's and RE's are similar. He mentions that these experiences have caused people to abandon jobs and devout their lives towards meaningful work such as charitable causes while also becoming religious or spiritual as a result (Whitehead, 2011). Resulting characteristics of this experience include: (1) an enhanced tolerance of others, (2) a spontaneity and energy towards life, and (3) being less fearful and less dependent on external supports for self-esteem fulfillment (Whitehead, 2011). 


\section{Psychopathological Experiences}

Accounts of psychopathology can be traced to religious and transcendental experiences of the Middle Ages (Kroll \& Bacrach, 1982). Due to the majority of schizophrenia symptoms in medieval Europe being explained through religious phenomena, mental illness was related to religious and supernatural experiences (Jeste et al., 1985). Similarly, Peters and Price Williams (1980) note that cultural conceptualization of religious or psychotic phenomena are important to consider due to similar mental and behavioural states being classified as psychiatric disorders in some cultural settings and religious experiences in others.

The development of psychopathology also finds its roots in the hysteria and spirit possession of the 19th century (Winkelman, 2010). Considered to be an involuntary encounter, possession by a spirit was comprised of the person being possessed, the family and the community who surrounded the individual, as well as the theologians who performed the exorcism. Being required for the successful removal of the spirit, the theologian had the authority to authenticate the possession (Di Agresti, 1980 as cited in Sluhovsky, 2011). As the phenomenon of spirit possession evolved, techniques of discerning spirit possession grew along with a literary genre that defined it (Anderson, 2002). However, despite these efforts, the spiritualization of demonic possession became a layered process that focused on the psychological rather than physical symptoms, changing its conceptualization (Sluhovsky, 2011). Thus, the pathology of spirit possession began to take shape.

Characterized as a disruption or disengagement from waking reality, psychopathological states include symptoms of dissociation, auditory and visual hallucinations, disorganized thinking and behaviour, depersonalization (loss of identity; a feeling of unreality) and derealisation (the awareness that you are observing yourself from outside your body) (American Psychiatric Association, 2013). The most common examples include psychotic disorders such as 
schizophrenia, Post Traumatic Stress Disorder (PTSD) related disorders and dissociative disorders (American Psychological Association, 2013).

According to Tart (1975) a transition from one state of consciousness to another can produce a temporary psychophysiological disorganization such as in Dissociative Identity Disorder (DID) where the individual can fluctuate between separate ego states (Courtois, Ford, Herman \& Van der Kolk, 2009). As well, people with PTSD can cycle between highly aroused states triggered by various stimuli while also becoming subjectively detached to their outward surroundings (Courtois, Ford, Courtois, Herman \& van der Kolk, 2009).

As mentioned above, Cardeña (2011a) suggests that alterations in consciousness due to trauma act as an induction procedure much like hypnotic induction does. In his review of the psychopathological state, Cardeña (2011b) suggests that lack of agency, or the inability to control parts of the whole body, reveal that an alteration in consciousness occurs. Individuals with mood disorders also experience stark changes in consciousness, displaying fluctuations in moods such as in depressive, anxiety and bipolar disorders (Cardeña, 2011b). As noted in the section on induction procedures, Vaitl et al. (2005) offered categorical distinctions of the various induction procedures including psychologically induced states occurring as a result of sensory deprivation, relaxation, and hypnosis and disease induced as seen in psychotic disorders, coma and epilepsy.

Research on psychopathology and the brain has revealed that dopamine and serotonin are implicated in psychotic disorders (Richtand \& McNamara, 2008), dissociative disorders and PTSD (Veltmeyer et al., 2006). As well, theta brain states have been seen to occur in psychotic (Richtand \& McNamara, 2008), dissociative (Manchester et al., 1988) and PTSD related disorders (Veltmeyer et al., 2006). 
Cardeña's literature review (2011b) reveals that individuals with borderline personality disorder (BPD), insecure attachment and trauma, experience an alteration in consciousness. In borderline cases, the extreme change in mania and hopelessness in relatively short time periods accounts for this alteration (Cardeña, 2011b). In his research, Cardeña (2011b) refers to Menezes Jr. and Moreira-Almeida (2010) who consider diagnostic differences between spiritual emergence versus spiritual emergencies. The former unfolds over time and does not pose any serious issues with psychological functioning, whereas the latter can have adverse effects on the psychological, social, and occupational domains of the individual (Grof \& Grof, 1989). Insecure attachment and trauma affect the individual's ability to trust other people and the environment, producing an inability to form secure attachment relationships (Courtois, Ford, Herman \& van der Kolk, 2009). The origins of this are believed to occur in early caregiving relationships where key representations of the self, other and self in relation to the other are established (Cook et al., 2005). The characteristics with insecure attachment include: (1) problems with boundaries, (2) lack of trust and suspicion, (3) social isolation, (4) interpersonal issues, (5) issues identifying with other people's emotional states, and (6) issues with establishing identity and sense of self (Cook et al., 2005). Thus, when the source of trauma is found in the initial caregiver relationship, the attachment ability of the individual is dramatically altered (Cook et al., 2005). When this occurs, it is estimated that $80 \%$ of maltreated children become insecurely attached (Friedrich, 2002). As a result, individuals with chronic trauma generally exhibit moderate to extreme dissociation (Steele, van der Hart, \& Niejenhuis, 2001). Dissociation includes experiences of: (1) a distinctive alteration in consciousness, (2) amnesia, (3) symptoms of depersonalization and derealisation, (4) two or more identifiable states of consciousness, and (5) an impaired memory of dissociative events (Cook et al., 2005). 
Despite dissociative experiences being linked to psychopathology, Krippner's (1999) account of dissociative experiences is important as it offers the reader a model that includes transpersonal perspectives. Krippner's (1999) model suggests that dissociative features do not necessarily indicate a psychological disturbance or disorder. As will be noted below in Figure 3, Krippner (1999) offers the reader the idea of "controlled dissociation" versus "uncontrolled dissociation" (p. 83). Dr, Krippner has allowed for the reprinting of this table and was contacted June 26, 2018. His definition of dissociation is descriptive and refers to an experience that is disconnected or apart from the usual flow of conscious awareness, behavioral repertoire, and/or self-identity (Krippner, 1999). Similarly, in their review of mental disorders of religious content and spiritual experiences, Menezes and Moreira-Almeida (2009) offered three criteria to support that spiritual experiences could be considered non-pathological. They include: (1) the absence of psychological suffering, (2) no social and occupational problems resulting from the experience and (3) the ability to interpret the uniqueness of the experience. Also, having the ability to control the experience allows for a positive and social outcome leaving no psychiatric comorbidity (Menezes \& Moreira-Almeida, 2009).

The first aspect of Krippner's (1999) model distinguishes between controlled flow, uncontrolled flow, controlled dissociation and uncontrolled dissociation. For example, controlled flow is analogous to an individual immersed in a process of concentration. Uncontrolled flow occurs when one is spontaneously affected by an event such as a beautiful sunrise over a mountain range. Controlled dissociation occurs when a shaman is in contact with a spirit for the purpose of helping others, and uncontrolled dissociation occurs when an individual suffering from a psychological disorder is swept over by emotional content that renders them unable to control their immediate disposition. The second aspect postulates if there are alterations in one's identification with the ego-self or if it is transcended contacting a hypothetical All-Self (divine, 
experience of oneness). Finally, the third aspect asks if this is life-affirming or life-denying. Krippner notes that the quality of the dissociation is determined culturally. Thus, in monophasic cultures such as the West (where logical and rational dominate the conscious experience) it is thought to indicate psychological dysfunction. While also being applied to voluntary "spirit incorporation" (p. 88) in polyphasic cultures (where the ASC is valued and considered as part of the conscious experience) experiences can occur as frequently as once or twice a week (Krippner, 1999). 
Figure 2

Model of Dissociative Experiences:

Control and Lack of Control, Flow and Interrupted Flow

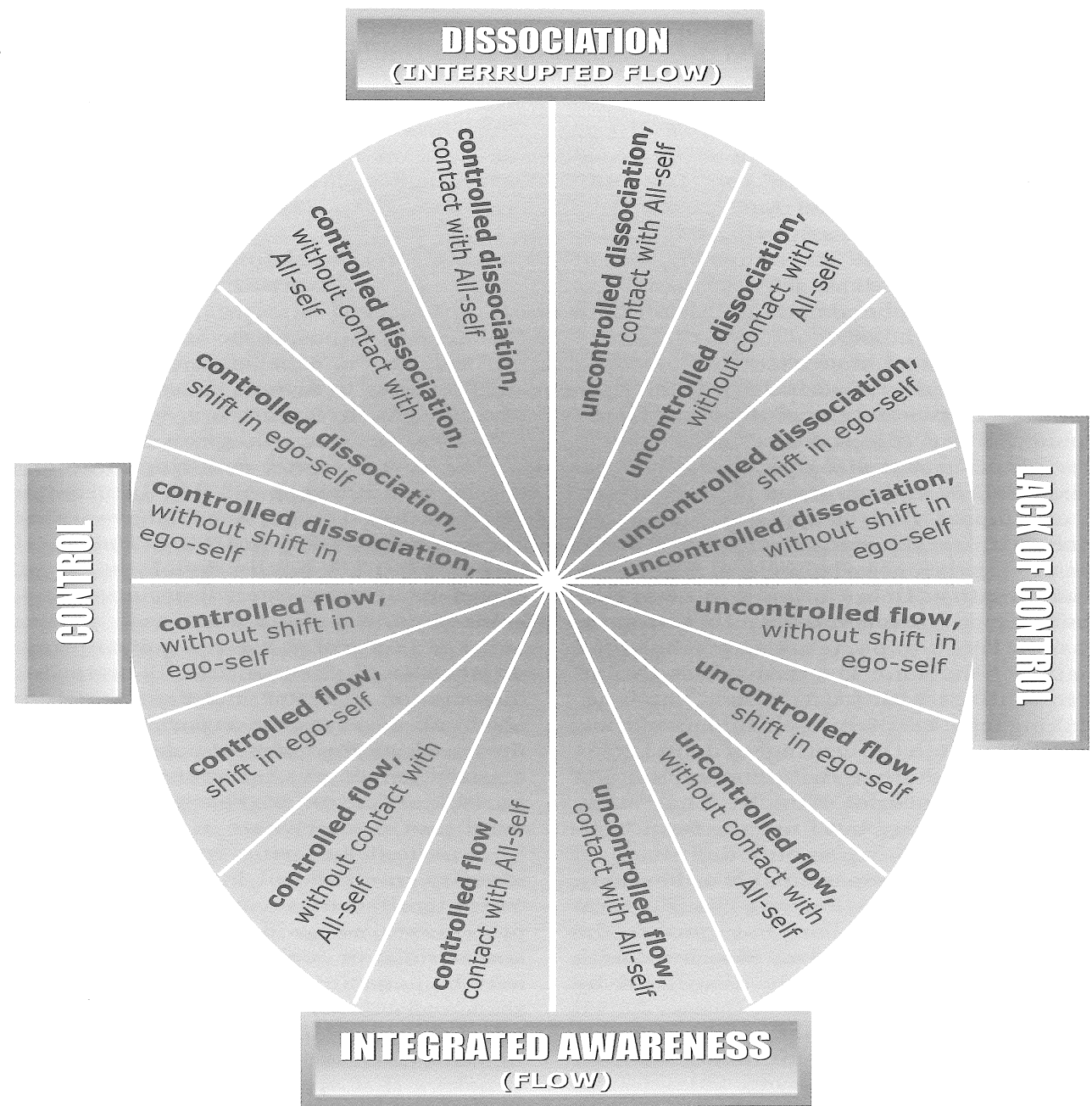

Note. Shading indicates that experiences are on a continuum rather than discrete.

84 The International Journal of Transpersonal Studies, 1999, Vol. 18, No. 2

Figure 4. Krippner's model of Dissociative Experiences. Reproduced by permission of Krippner, S. (1999, p. 84) on June 26, 2018). Please see Appendix A for the email that granted the use of Figure 4. 
Finally, Cardeña (2011b) notes that the ASC is part of the human experience that includes pathology and health. Since one of therapies goals is to adjust the client's current conscious state into a more cohesive structure, clinical psychology and psychiatry should focus on the ASC to understand the specific forms of pathology where the ASC manifests (Putman, 2005 as cited in Cardeña, 2011b). Thus disorders such as BPD, PTSD and schizophrenia qualify as an ASC due to noticeable changes in perception occurring such as dissociation, auditory and visual hallucinations, disorganized thinking and behaviour, depersonalization (loss of identity; a feeling of unreality) and derealisation (the awareness that you are observing yourself from outside your body) (American Psychiatric Association, 2013).

Now that a thorough understanding of the religious, spiritual, transpersonal and psychopathological experiences has been outlined, we now turn our attention to the Shaman's Altered State of Consciousness where aspects of religious, spiritual, transpersonal and psychopathological experiences have been associated with the shaman (Devereux, 1961; Walsh, 2007; Winkelman, 2010).

\section{The Shaman's Altered State of Consciousness}

Although preliminary research on the ASC has questioned the mental health of the shaman (Devereux, 1961; Silverman, 1967), it has been discredited based on the impact that Western viewpoints had on the specific cultural practices of the shaman (Noll, 1983). As well, the commonalities seen in cross-cultural occurrence of the ASC has produced the following characteristics common to the experience. They include: (1) a subjective experience (Fisher, 1986; Tart, 1975), (2) an induction method (Fisher, 1986; Tart, 1975), (3) an alteration in physiological and psychological awareness (Ludwig, 1966), (4) an alteration in logic (5) an alteration of time; (6) a noticeable change in perception; and (7) a sense of joy and awe (Tart, 1975). Since the shaman comes from a polyphasic culture (cultures that value the ASC as part of 
the cultural experience; Laughlin et al., 1990), the shaman's emersion and training in the ASC is the primary focus, allowing for control and mastery of the ASC to evolve (Noll, 1983;

Winkelman, 2011). Understanding this, researchers' interest in the shaman's ability to utilize the ASC has produced phenomenological investigations (Walsh, 2007;1995), ethnographical (Eliade, 1951/2004; Winkelman, 1986, 2010) and descriptive accounts of the shaman's ASC (Krippner, 2009; VanPool, 2009).

What follows is a review of the shaman's ASC from key researchers in the field.

Distinctions and similarities will be highlighted with an informed definition being offered afterwards. Specifically, the work of Michael Harner (1980/1990) distinctions made by Peters and Price Williams (1980), Clottes and Lewis-Williams (1998), Krippner (2000) and Van Pool (2009) will be noted. The Integrative Mode of Consciousness (IMC) theory by Michael Winkelman (2010) will be highlighted and finally, Walsh's (2007) ten characteristics of the shaman's ASC will be described. For clarity, this review will adopt Harner's (1980/1990) term to describe the shaman's ASC known as: the shamanic state of consciousness (SSC).

\section{Michael Harner and the SSC}

In his work, Harner (1980/1990) identified that the shaman experiences two distinct types of consciousness; the ordinary state of consciousness (normal waking reality) and non-ordinary reality (NOR) otherwise known as the ASC. Determining that the NOR is of a different landscape, Harner (1980/1990) concludes that the ASC of the shaman is induced by the NOR experience. Identifying this as the shamanic state of consciousness (SSC), Harner (1980/1990) notes that the shaman enters the NOR to communicate with spirits who give specific knowledge and information to the shaman for healing purposes. Harner (1980/1990) mentions that the basic tools to access the SSC occurs from the use of the drum and the rattle, and through chanting. Being fundamental to achieving the SSC, the drum is used across shamanic culture (Harner, 
1980/1990). The shaking of the rattle offers further stimulation to higher frequency states in the brain reinforcing the drum beats and further increase of the sonic effect (Harner, 1980/1990). As well, Harner (1980/1990) concludes that many shamanic practices use chanting to evoke the SSC. Usually repetitive and monotonous, chanting encourages the shaman's guardian spirits and helpers to be present, assisting the shaman with the maintenance of his power (Harner, 1980/1990).

According to Eliade (1951/2004), the drum has complex symbolism and a variety of uses, with its iconography representing the symbolism of the ecstatic journey. Whether it takes the shaman to the "center of the world", into the air, or summoning spirits to the shaman's aid, the shaman uses the drum to establish contact with the spiritual world (Eliade, 1951/2004, p. 168). Harner and Tyron (1992) emphasize that rhythmic drumming encourages a theta brain wave induction. Similarly, work done by Previc (2010) acknowledges this noting that rhythmic drumming deliberately excites the hippocampal theta waves that occur during REM sleep, meditation and in orientating distant stimuli.

Harner (1980/1990) mentions further that the SSC allows the shaman to "see" shamanically (ability to understand the information gathered in the SSC) and occurs as a fluctuating range of states. Ranging from light to very deep, the shaman uses the SSC according to what it is required for (Harner, 1980/1990). Due to the importance that the ASC has on polyphasic cultures, the interactions that occur while induced in the SSC such as those with animals, humans or other phenomena are considered to be an authentic experience (Harner, 1980/1990). Thus, when the shaman is fully immersed into the SSC it is acknowledged it as his/her "immediate reality" (Harner, 1980/1990, p. 53).

Finally, Harner (19801990) emphasizes that the successful use of the SSC requires a deep respect and awareness for all forms of life with the realization that we all depend on each other 
for survival. Understanding that humans are related to all forms of life, the shaman approaches other life forms with an unconditional respect and understanding (Harner, 1980/1990).

Research offered by Peters and Price-Williams (1980) who compared 42 societies from North and South America, Africa and Caribbean, North and South Asia, South East Asia and Oceania, identified three similarities among the SSC: (1) voluntary control of entrance and duration of the altered state, (2) ability to communicate with others (spirits, ancestors), and (3) a memory of the experience. Furthermore, shamans in eighteen of the cultures reviewed by the authors discussed spirit possession: (1) Chukchee, (2) Altaic, (3) (4) Yakut, (5) Tungus, (6) Evenks, (7) Pahari, (8) Senapur, (9) Mysore, (10) Tikopia, (11) Batak, (12) Sea Dyak, (13) Semang, (14) Mentawai, (15) Sambia, (16) Veddes, (17) Niue, and (18) Burma; with ten having out of body experiences, eleven showing both and three having a different altered state (Peter, Price-Williams, 1980).

Research done by Clottes and Lewis-Williams (1998) suggested that three stages exist to the SSC: (1) light alteration of consciousness whereby experiences of geometric forms and meandering lines are experienced; (2) complex meaning being made to these experiences; and (3) a synthesis of the information being gathered in the SSC and application to the individual being treated. Taking both individual experience and social understanding into consideration, Krippner (2000) suggests that shamans rely on information from a data base consisted of dreams, visions, intuitions and personal observation of the natural and social world. In their conclusions, both Sansonese (1994) and Krippner (2000) suggest that a genetic pre-disposition may exist amongst shamans using the SSC. To support this claim, Krippner (2000) concludes that the tradition of the Indo-European shaman utilized a parent-to-child transmission of knowledge that established extended-family shaman groups. This can be seen in the Huichol Indians of Mexico whose myths, songs and formal speech contains the knowledge and experience of extensive 
peyote use (Eger, 1978 as cited in Schaefer, 2011). Handed down as stories, myths and rituals to the children of the culture, this knowledge acts as key cultural symbols entrenching the identity and worldview into its predecessors (Schaefer, 2011).

In her research, VanPool (2009) mentions that the shaman can interact with spirits during waking or dreaming while also being able to transform into one. Achieved through rituals that induce the shaman into the SSC, the distinction rests with the SSC being a supernatural encounter that exists within the specific cultural framework (VanPool, 2009). VanPool (2009) notes that these supernatural encounters occur in the spirit world. As a result, VanPool (2009) concludes that this information lends to the importance of a unifying definition of shamanism that should include the SSC as a distinct ASC that is based completely on the cultural framework, ideology and belief system of the shaman.

Other researchers, such as Noll (1983) states that the SSC is entered willingly, defining it as a visionary state marked with spontaneous imagery and vivid lucidity. Noll (1983) concludes that the defining feature of the shamanic altered state is twofold: (1) involving the active engagement of the shaman, and (2) the shaman's control of the experience. However, Noll (1983) does not specify what control refers to. Peters (1982) further explains that the shaman is the participant, observer and controller of the altered state.

\section{The Integrated Mode of Consciousness}

From his cross-cultural research, Winkelman (2010) argues for an integrated framework to describe the activities of shamanism globally. The core of Winkelman's (2010) IMC theory is based upon: (1) cross cultural studies that suggest ASC is a worldwide occurrence (Bourguignon, 1968; as cited in Bourguignon, 1989; Eliade, 1951/2004); (2) the application of the dopamine theory (Previc, 2011) whereby the evolution of dopamine has contributed to the ability of the shaman to utilize the ASC and; (3) Maclean's triune brain model (1973) who suggested that 
specific behavioural and psychological functions of the ASC are located in the reptilian, paleomammalian and mammalian brain structures (Winkelman, 2010). With cross-cultural studies on shamanism having been already outlined, the review will focus on the importance that the dopamine theory and Maclean's triune brain theory (1973) has on Winkelman's IMC theory (Winkelman, 2010).

\section{Dopamine, the Brain and ASC}

The release of dopamine in the body produces a pleasurable response helping form memories to induce the experience of gratification (Salamone \& Correa, 2002 as cited in Winkelman, 2010). Examples of this are seen in the following: (1) eating and sexual activity, and (2) recreational drug use of caffeine, nicotine, THC and in psychedelics (Previc, 2011).

In his review Previc (2011), emphasizes the importance that dopamine has had in human evolution. Previc (2011) notes that the human brain has mostly evolved from epigenetic factors or external influences that can override genetic expression. Previc's (2011) review of the literature concludes elevation in dopamine levels in humans occurred during the emergence of Homo Habilis over two million years ago, and the emergence of the modern human around eighty thousand years ago. Dopamine is implicated in neurological processes such as reward prediction, stimulus associations, cognitive abilities and biological processes such as lowering blood pressure (Winkelman, 2010; Previc, 2011). Examples of this occurring across culture are seen in the development of making stone tools and enhanced running to attain meat for dietary purposes (Previc, 2011). According to Previc (2011), a determining factor of dopamine increase in early human populations was the inclusion of meat into the diet. Due to meat having abundant sources of tyrosine, it allows for improved thermal tolerance and endurance capabilities and acts as a precursor to dopamine enhancement (Gilbert, 1995 as cited in Previc, 2011). Previc (2009) also suggests that the addition of marine fauna into the diet of humans provided essential amino 
acids and iodine that boosted dopamine transmission and improved human longevity (Previc, 2011).

Previc (2011) also notes that a common feature of all ASC's is a disinhibiting of dopamine that occurs in the extra personal brain system located in the ventral cortex, the area known for playing an important role in consciousness (Previc, 2009 as cited in Previc, 2011). For example, mystical experiences, dreams, out of body experiences, floating sensations, deep hypnosis and hallucinations are all thought to occur in ventral brain pathways (Cardeña, 2005; Girand, Martius, \& Cheynes, 2007; as cited in Previc, 2011). Dopamine also plays a key role in parasympathetic influence on bodily functions and inhibiting factors in sympathetic emotional arousal systems in the amygdala and posterior cortex of the brain (Harris, 2005 as cited in Previc, 2011). Parasympathetic dominance has been associated with out-of-body experiences and other altered states such as meditation, drug use, prolonged dancing and trauma (Mandell, 1980; Sierra \& Berrios, 1998; Winkelman, 2010).

\section{The Triune Brain Theory}

The triune brain theory of Maclean (1973) discusses the integration of the reptilian, mammalian and paleo-mammalian brains as evidence for an ASC (as cited in Winkelman, 2010). As mentioned in Note 1, the reptilian brain regulates metabolism, digestion, and respiration and is responsible for wakefulness, attentional mechanisms and regulates coordination of behaviour (Winkelman, 2010). The mammalian brain is responsible for the regulation and control of bodily activities, as well as receiving and interpreting sensory impulses and transmitting information to the muscles and organs (Mammalian, n.d.). Finally, the paleo-mammalian brain is responsible for motivation, emotion involved in feeding, reproductive behaviour and parental behaviour (Paleo-mammalian, n.d.). Thus, when integrated, a cohesive hierarchy of information processing results, allowing for altered states to occur (Winkelman, 2010). Winkelman (2010) applies 
Maclean's theory stating that the SSC of the shaman produces psychophysiological and psychosocial brain integration by boosting interaction between conscious and unconscious processes manifesting as visions for the shaman. Furthermore, the triune brain model helps explain alterations of consciousness by offering a foundation that helps explain the nature of shamanism and the effects that the SSC has (Maclean, 1973 as cited in Winkelman, 2010).

In his ethnographic review of shamanism Winkelman (2010) identifies three defining aspects of the SSC: (1) shamanic soul flight; (2) meditative absorption (assimilation; incorporation); and (3) mediumistic possession. Soul flight is the ability of the shaman to leave his body during the SSC enabling him to obtain the necessary information required for helping those involved (Winkelman, 2010). Produced by preparatory restrictions such as fasting and followed by excessive chanting, drumming and extreme physical behaviour (dancing), the shaman eventually collapses and enters into the SSC (Winkelman, 2010).

Created by an internal focus, meditative absorption is achieved by chanting or drumming as well as sleep deprivation or fasting (Winkelman, 2010). As well, the shaman can be induced into mediumistic possession that manifests as tremors, convulsions or amnesia (Winkelman, 2010). This occurs when the shaman is exposed to rituals involving singing, chanting or percussive music (Winkelman, 2010). According to Winkelman (2010) the SSC has similar patterns to that of sexual activity producing a sympathetic activation that is followed by a parapsympathetic-dominant state.

Winkelman (2010) notes that shamanic and mystical traditions encourage the development of an inner perception to be formed. This gives the shaman the ability to navigate the SSC allowing the shaman to perceive spirits and to navigate the spirit world. Noting that this ability is biologically based, Winkelman (2010) argues that this biological framework has developed into underlying inherent structures that are formed through a cultural understanding of 
myth, such as belief in spirits and healing. As a result, Winkelman (2010) suggests that they are reinforced through rituals to achieve cognitive integration and psychological understanding.

Further to his research, Winkelman (2010) mentions that shamanic ritual creates a range of subjective experiences that need to be addressed in a comprehensive theory of consciousness. This can be seen in the shaman's ability to identify the discrete changes in consciousness that lead into the SSC (Winkelman, 2010). For example, the psychophysiological and experiential changes created by shamanic activities affect daily sleep/wake cycles, dreaming, deep sleep and what he calls the transpersonal (aspects of consciousness that extend beyond an individual's body and ego), spiritual (a subjective, intuitive, holistic, tactile experience involving nonverbal aspects of perception and knowledge), or transcendental consciousness (specific conditions defined by their own intrinsic properties). Furthermore, the SSC displays a uniqueness that is different from ordinary waking consciousness so much that it offers research insight into the fundamental nature of consciousness (Winkelman, 2010).

Finally, Winkelman (2010) explains that symbolic meaning is crucial to the SSC as it acts as a reference that helps interpret outward stimuli. Thus, symbols are incorporated into the biology of the individual through personal and cultural experiences (Winkelman, 2010). Winkelman's (2010) findings support Jung's theory of the archetype whereby the archetype is manifested into the person's daily life as a symbol to encourage integration and understanding to occur (Jung, 1980/1959a). What follows now is Walsh's ten dimensions of the SSC.

\section{Ten Dimensions of the SSC}

Basing his findings on previous research (Eliade, 1951/2004; Noll, 1983; Peter and PriceWilliams, 1980) as well on interviews with shaman apprentices in South America and of the Huichol and Nepalese traditions, Walsh's (2007) research helped clarify the criteria associated with the SSC. As a result, Walsh (2007) suggests that ten key dimensions are required for 
defining the SSC. Also, known as the shamanic journey, these include: (1) degree of control (over the content of the experience), (2) awareness of the environment, (3) ability to communicate, (4) concentration, (5) degree of energy or arousal, (6) degree of calm, (7) emotion, (8) sense of identity, (9) out of body experience (OBE) and, (10) the nature of inner experience. As Walsh (2007) notes, a distinguishing feature of a shaman is his ability to control the SSC, eventually leaving and entering it at will. Requiring an extensive amount of training and induction into the SSC, mastery occurs over a period of time (Harner, 1980/1990). Although obtaining control is paramount to entering and leaving the SSC, Walsh (2007) notes that what the shaman experiences while induced into the SSC changes each time. Thus, the shaman uses his previous knowledge of the experience to interpret what is being perceived (Walsh, 2007). Due to the shaman being induced into the SSC, Walsh (2007) notes the shaman's awareness of the physical environment is lessened during this experience because of his attention being placed on the SSC. This allows for the shaman to communicate the information he has received for the individual or community he is serving (Walsh, 2007).

As noted by Walsh (2007) concentration, energy arousal, a deep sense of peace and a variety emotion are often experienced by the shaman. Determined through concentration, the shaman's focus is a trait that is required for successful navigation of SSC (Walsh, 2007). As well, the shaman may feel energized with a deep sense of peace occurring during the SSC. Emotions may vary from dread to despair, to pleasures and excitement, with a similar range of emotions occurring after the experience ranging from fatigue to depletion (Walsh, 2007). With reference to the OBE, and the nature of the inner experience, Eliade (1951/2004) describes the OBE as an "ecstatic" experience with the shaman perceiving himself as a disembodied spirit with the ability to navigate far distances at tremendous speed. According to Walsh $(2007$, p. 242$)$ the nature of the shaman's inner experience is "rich, complex, and multisensory." Also, because of 
his ability to remain conscious during the SSC (Walsh, 2007) the shaman has a total recall of the experience.

In summary, advances in ASC research has considered the shaman's ASC to be unique, thus classifying it as the SSC. Furthermore, the shaman is referred to by the literature as an individual who has obtained mastery and control over the SSC (Harner, 1990; Peters and Price Williams (1980), Walsh, 2007; Winkelman, 2010). As well, similarities observed in the shaman from Winkelman (2010) and Harner (1980/1990) include; (1) the ability to visualize, interpret and incorporate information from the SSC, (2) the innate understanding of navigating the SSC; and (3) the use of chanting and or/drumming to evoke the SSC. Also achieved through induction procedures such as ingesting psychoactive compounds (Winkelman, 2011) the SSC has been described as a fluctuating range of states (Harner, 1980/1990), light alterations of consciousness (Clottes \& Lewis-Williams, 1998), and is obtained by hereditary transmissions through story, ritual and mythology (Krippner, 2000). Finally, Walsh's (2007) ten characteristics of the SSC has allowed for clarity and consensus on the experience. Specifically, Walsh's (2007) researcher clarified that the shaman's experience with the SSC changes each time, confirming that mastery and control are essential characteristics to the shaman's success.

Therefore a definition of the SSC includes the following criteria: (1) induction procedures such as drumming/chanting and the use of psychoactive compounds; (2) control/mastery over time; (3) experiencing the SSC from a light fluctuation to a full emersion state, (4) experience of communicating with spirits of human, animal, or discarnate forms; and the (5) ability to visualize, interpret and incorporate information from the SSC (Clottes \& Lewis-Williams, 1998; Harner, 1980/1990; Walsh, 2007; Winkelman, 2010).

This chapter has offered an overview of the consciousness and altered consciousness (ASC) experience from western accounts to research on the shamanic state of consciousness. It 
has put forward the easy problem of studying consciousness with research identifying brain regions that are associated with the consciousness experience (Rees \& Frith, 2007; Velmans, 2007), as well as the hard problem of studying consciousness where research cannot answer the question as to why we are conscious (Cárdeña, 2011a; Chalmers, 2011). Despite advances in consciousness research combining subjective accounts with objective data on consciousness (Beauregard, 2007; 2014, Haynes and Rees, 2005; Rees and Frith, 2007; Velmans, 2007) using objective data to establish a science of explaining consciousness has remained (Velmans, 2007). Thus, the problems that psychology and disciplines such as neuroscience face with explaining why we are conscious persist and the issues with forming a science of consciousness endures due to how its contents are explained (Velmans, 2007).

Finally, by recalling Fischer (1981) who concluded that our logical reasoning can strongly influence our perception, it suggests that our experience of consciousness over time is shaped by our perception of reality. Thus, Fisher's (1981) research implies that the universe as we understand it is not the real universe, rather a model to refer to. Similarly, at the Quantum level of understanding, researcher indicates that the interplay of reality is heavily influenced by perception (Wolf, 2001). Thus, if our perception of life has been heavily influenced by the cultural influences we have employed (Fisher, 1981; Whitehead, 2011; Wilbur, 1977/1993), then it begs the question of what consciousness really is.

What follows now is the methodology of the study, with the results and discussion to conclude the thesis. 


\section{CHAPTER V}

\section{METHOD OF THE QUALITATIVE STUDY}

\section{Purpose}

The purpose of this phenomenological study is to describe the shamans' experience of the ASC at the physical, cognitive, emotional and spiritual level (Harner, 1980/1990). Creswell (2013) suggests that a minimum of 3 and a maximum of 15 are suggested for an adequate group of participants. Using a phenomenological focus, this study investigated the lived experience of the ASC in 5 shamans. Phenomenological research is comprised of a level of focused research activities and is often used in the social and health sciences, particularly in sociology, psychology, nursing and education (Creswell, 2013). It combines a set of exploratory, material practices that make the world visible, thus allowing for a transformation of the world to take shape (Creswell, 2013).

The goal of phenomenological research is to acquire an appreciation of the phenomena according to the understanding people bring to them (Creswell, 2013). van Manen (1990) suggests that the purpose behind phenomenology is to bring out the original understanding of what is being studied. The inner drive that allows this to occur is: pathos (van Manen, 1990). Pathos originates from the Greek word, paschein, and is described as an element in experience or in artistic illustration inducing pity or compassion (www.meriam-webster.com). It leads the researcher towards the knowledge behind the experience of the phenomenon (van Manen, 1990). This creates an understanding of the essence behind the phenomenon, bringing the practical aspects of the experience to life, thus revealing why the experience is important (van Manen, 1990). It is the essence of what is being looked at, the original source of what makes it significant (van Manen, 1990). 
Phenomenology looks at a phenomenon and isolates its original essence by understanding how it fits into the overall experience of consciousness. Guba and Lincoln (1988) identify four philosophical assumptions behind effective phenomenological research. These include: (1) Ontology; (2) Epistemology; (3) Axiology; and (4) Methodology. Ontology considers the nature of reality and its defining characteristics. The researcher uses ontology by embracing the idea that multiple realities exist. Using an ontological lens gives the researcher the ability to use the exact phrases and words the participants use in order to capture the multiple realities that exist between each participant (Moustakas, 1994).

Using the philosophical base of epistemology, the researcher attempted to get as close as possible to the participants gathering knowledge from a subjective viewpoint and assembling it based on individual narratives (Creswell, 2013). Thus, the data on the shamans' experience of the ASC can only be grasped fully if it is gathered from the participants' environment (Creswell, 2013). Axiology considers the role of values in research. Thus, the researcher has to make his own values known in the study through a process of transparency, and allows to report his values and biases along with the information accumulated from the field (Creswell, 2013). The researcher made each participant aware of his personal interest and involvement in shamanism prior to the study. By taking this into account, the researcher offered a critical analysis of the literature by highlighting commonalities as well as differences in shamanism. As well, the interview questions and process outlined by Seidman (2013) were chosen to allow for a general to a specific focus to occur to ensure that the essence of the phenomenon revealed itself.

A defining feature of phenomenological research is the inductive process of gathering data (Creswell, 2013). Thus, information emerges and is shaped by the researcher's experience in collecting and understanding the data (Creswell, 2013). The data is driven by the axiological assumption of the researcher (researcher's experience) however its collection is accomplished 
while immersed in the field, thus is done from the "ground" up (Creswell, 2013). This scenario allows for flexibility and changes to be made during the research process as new data can be discovered (Creswell, 2013).

Phenomenology can also take a broader reach by addressing the impact that phenomena have on our conscious experience. Thus, the significance of objects, events, the experience of time, self or others, are also considered in phenomenology (Creswell, 2013). By understanding phenomena according to the subjective understanding people bring to them phenomenological research begins with assumptions using an interpretive/theoretical framework. This framework uncovers the essence of what individuals or groups experience according to the phenomena being studied (Creswell, 2013, Moustakas, 1994). Phenomenology focuses on gaining understanding with the reflective process being a key factor in phenomenological research. It involves discernment by considering how we understand the sensation of the world around us (Creswell, 2013; Moustakas, 1994). Furthermore, by using the phenomenological approach, it uncovers the natural process of the phenomenon, adding to theoretical development and contributes to quantitative research by offering the essence behind the phenomena being studied (Creswell, 2013).

\section{Objectives of the Study}

The objectives of the study included: (1) bring clarity to the ASC experience of the shaman, (2) focus on the shaman in North America.

\section{Research Questions}

1) What are the set of described experiences that the shaman has while induced into the altered state of consciousness.

a) How does the shaman describe his experience on a physical level?

b) How does the shaman describe his experience on an emotional level? 
c) How does the shaman describe his experience on a spiritual level?

d) How does the shaman describe his experience on a cognitive level?

\section{Philosophy}

The exploratory framework of phenomenology is found in philosophy (Creswell, 2013). Using a philosophical base helps the researcher understand how to establish the research questions and how to uncover the information that will answer them (Creswell, 2013). For example, the writings of Edmund Husserl, Heidegger and Sartre (Spiegelberg, 1982 as cited in Creswell, 2013), have contributed to phenomenological inquiry by asking fundamental question about the nature of phenomena.

\section{Husserl}

In his conclusions, Husserl suggests that knowledge can only be accepted if it is obtained from the immediate experience as phenomenology does not concern itself with facts; it seeks to establish itself as a "science of essences" (van Manen, 1990). Husserl (1960/1982) urges the reader to access an inner wisdom that invites original insights that inform the individual of his/her immediate experience. This must begin in an "absolute poverty" to ensure that the philosophical inquiry begins with a "lack of knowledge" (pg. 2).

Husserl was interested in the importance behind the essence of the phenomena being studied as it appeared through consciousness and concluded that both mind and objects occur with experience (Laverty, 2003). Husserl (1960/1982) suggested that clarity occurs with a total immersion into the phenomenon being studied, allowing for all the aspects of the experience to become known. Thus, consciousness was a co-occurring event between the person and the world and accessing consciousness was achieved through the direct grasping of the phenomena, via intentionality (Laverty, 2003; van Manen, 1990). According to Husserl, the process of 
intentionality is comprised of the mind being directed towards objects of study allowing for a description of the phenomena to take shape (Laverty, 2003; van Manen, 1990).

An important aspect of Husserl's work was the process of bracketing, or phenomenological reduction. Bracketing is the process of eliminating bias (collective and individual) to arrive at the experience of the phenomena by using participant data or the researcher's in order to understand the essence of the phenomena (Laverty, 2003). Husserl (1960/1982) discusses this as "grounding judgement" (pg. 11), allowing no judgement to be accepted until the researcher has understood it and can justify it by the observations made. Therefore, by suspending judgement of the phenomena being studied, the researcher allows for the possibility of the meaning behind the phenomena to emerge (Laverty, 2003). Klein and Westcott (1994) understand it as a 3-fold process by using: (1) exemplary intuition (holding phenomena in imagination); (2) imaginative variation (uses this to develop examples of similar experiences using imagination), and (3) synthesis (integrating both to arrive at the essence; Laverty, 2003). Husserl notes (1960/1982) that by immersing oneself into the phenomenon being studied, one uncovers essential aspects to the understanding of the phenomenon.

\section{Heidegger}

According to Laverty (2003) Heidegger was motivated by Aristotle and considered two fundamental questions: (1) what brings our conscious experience together? And, (2) what is it that unites our Being-ness or existence? Being fully aware that we take this for granted Heidegger's main question that drove his pathos was: If we know that things exist then: What does "to exist" mean (Laverty, 2003)? This later became known as Hermeneutic phenomenology as it concerned itself with the lived experience of being human (Laverty, 2003).

Hermeneutic phenomenology considers how life is lived and focuses on highlighting the flippant details of human experience in order to establish meaning and a sense of understanding 
(Wilson \& Hutchinson, 1991). The main difference between Husserl and Heidegger was Heidegger's focus on Dasein: 'the mode of being human' or 'the situated meaning of a human in the world' (Laverty, 2003, p. 24). Due to this consciousness was not separate from the world it is formed by our historical experience (Heidegger, 1927/1962 as cited in Laverty, 2003). Further to Heidegger's notion was the undividable link between a person's background and the world allowing for a pre-understanding of culturally entrenched ideas to exist within the person as they evolved through life (Koch, 1996). Thus, Heidegger approached his phenomenology by looking at human cultural expression. This was accomplished by gathering information from written, verbal, visual and expressive arts such as music or dance with the notion of interpreting it to discover the intended or expressed meaning of the experience (Kvale, 2009).

\section{Sartre}

Expanding on the inner and outer conscious experience, a main focus of Sartre was his rejection of the Cartesian view of reality. Sartre furthered his criticism by focusing on Heidegger's concepts of being into nothingness of which 2 concepts were central to his critique: (1) Being-in-itself, defining it as: outward things that are non-conscious, possessing an essence, existing independently of any observer comprising of all the things in the word, such as a tree. Secondly, (2) Being-for-itself was defined by Sartre as: those beings that are conscious, making them distinct from other things in relation to themselves and others (Sartre, 1943/2005). In the introduction to Being into nothingness, Warnock notes that Sartre's main objective was to explain the relationship between being-in-itself and being-for-itself (Sartre, 1943/2005). Sartre reflected that objects themselves appear firstly as what they solely represent, a distinct entity onto its own. This is the first awareness we come to in our ontological inquiry, the being of appearance. Upon further investigation, his inquiry led him to realize that the phenomenon being studied isn't what it appears to be (Sartre, 1943/2005). 
Criticizing Descartes notion of "I think therefore I am", Sartre (1960) argues that the "I am" is not the consciousness which thinks, rather a secondary activity is at work during this process. Furthermore, Sartre (1943/2005) understood that our conscious experience helps us realize that we are not the inner or outer experience we think we are. Rather, to be fully consciousness is to realize that we are both the perceiver of the object and the object being perceived. Sartre concluded that we are attempting to create our experience between our experience of ourselves (being-for-itself) and the outward experience of our self. By only being aware of ourselves when we encounter another robs us of our freedom because we are objectifying and are being objectified. Thus, we immediately identify with being-in-itself rather than with being-for-itself (Sartre, 1943/2005).

\section{Procedure}

This study incorporated the ideas of Husserl, Heidegger and Sartre by focusing on the phenomenon of the shaman's experience of the ASC. Thus, the study : (1) explored the ASC in a group of shamans; (2) attempted to bring out the original understanding of what is being studied (van Manen, 1990) and, (3) to outline the individual subjective experience of each shaman and consider the objective experiences of what the shaman participants have in common with each other (Creswell, 2013). The methodological approach included: (1) collection of the data through a 3-step interview process whereby each participant was interviewed 3 times. The interview begins from a general perspective and moves to a specific focus enabling the data to emerge naturally and the essence of the phenomenon (the experience of the ASC) to emerge, (2) transcribing the narratives; (3) reading the narratives; (4) regrouping the narratives into meaning units ensure the essence of the ASC is captured; (5) extracting emergent themes from the meaning units; and (6) using the information from the emergent themes to look for an overarching theme. 
The interview process suggested by Seidman (2013) is explained as follows: (1) the first interview is referred to as: focused life experience. The researcher's role is to put the participants' experience in context by asking them to discuss the details of their life history with the experience of the phenomenon being studied; (2) the second interview is referred to as: the details of the experience. The intention of the second interview is to focus on the tangible details of the participants' present lived experience, i.e. the participants are asked to reconstruct the details of this experience; (3) the third interview is referred to as: reflection on the meaning. During the third interview participants are asked to reflect on the meaning of their experience. Questions are asked to address the intellectual and emotional connections between the participants' work and life, i.e. they require the participants to look at how the situations in their lives interacted to bring them to their present understanding of the phenomenon. By exploring the combination of the past for clarification and what led them to describe the tangible experience of the phenomenon the interview creates the condition for reflecting upon what the participants are doing in their lives. According to Seidman (2013), the third interview can only be effective if the foundation for it has been established during the first two. He notes that 90 minutes is rationalized for each interview because an hour is too standard and causes participants to "watch the clock" (Seidman, 2013, p.23). Two hours seems too long and anything shorter than 90 minutes is too short because of the time it takes participants to reconstruct their lives. Finally, the length of time needs to be decided upon before the interviews because it gives a chronological order to them and it is suggested that each interview be spaced from 3 days to a week apart (Seidman, 2013).

Data was audio recorded using an adaptation of Seidman's (2013) 3-part semi-structured interview process, whereby the interviews were a day apart. This was rationalized due to the demand it may have put on the participants. 


\section{Organizing Data and Data Analysis}

Qualitative research gathers a large amount of data for interpretation (Creswell, 2013).

The data will be organized according to Creswell's (2013) template for coding a phenomenological study as displayed in Figure 5.

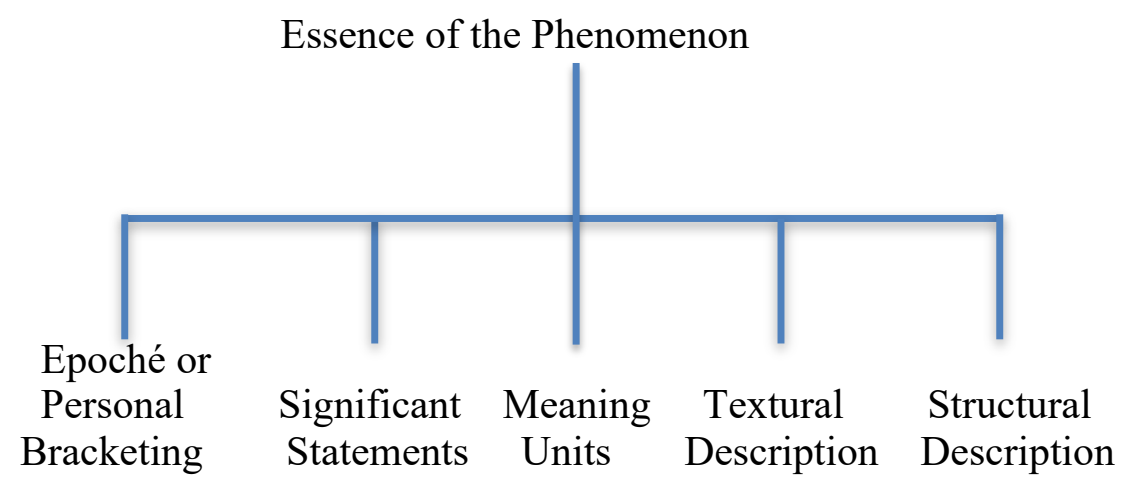

Figure 5. Template for Coding a Phenomenological Study (Creswell, 2013, p. 3925). Permission has been granted by Sage Publishing through Copyright Clearance Centre on September 26, 2018 via email. Please see Appendix A for the granting of the use of Figure 5.

From the participants experience of the ASC (the essence of the phenomena) the data was organized in the following manner: (1) through personal bracketing of the researcher's experience (setting aside preconceived experiencers of the participants in the study). According to Bevan (2014) the phenomenological reduction or epoché is a focused awareness that shifts the researcher out of his preconceived notions of the phenomenon, allowing the researcher to adopt a critical viewpoint. By adopting this stance, the researcher in this study questionned his own understanding of the phenomenon. This allowed for a new way of thinking, theorizing and experiencing the phenomenon to occur. Moustakas (1994) clarifies this further by suggesting that the researcher provides a detailed description of his own experience of the phenomenon so that the focus remains on the participant's experience. Secondly, (2) identifying significant 
statements from the audio-recorded interviews (Creswell, 2013), allowed for the text to be separated into identifiable meaning units. Thirdly, (3) the identifiable meaning units were regrouped into emergent themes that identify commonalities of the ASC experience across the participants'narratives. The data was then formulated into a (4) textural description of what the participants experienced with the phenomenon. Creswell (2013) notes it is important to capture the "what" of the experience in the participant's own setting. Finally, (5) a structural description of the experience was outlined to give an understanding of how the experience occurred according to the setting and context it was experienced (Creswell, 2013).

Giorgi (2009) describes the process whereby the researcher examines the data to get a sense of the whole picture of the phenomenon and from there determines the meaning units and then transforms the participant's description into psychologically sensitive expressions (as cited in Creswell, 2013). Thus, meaning units and emergent themes appear, eliminating overlapping and repetitive statements (Moustakas, 1994) and a description of the "essence" then emerges from this process (Creswell, 2013).

Therefore, during this study the researcher interviewed the shaman in his or her own natural setting because the site of the shaman is directly related to the world-view the shaman utilizes in his/her healing work (Winkelman, 2010, Walsh, 2007, Eliade, 2004). Since it is likely that the researcher absorbed the experiences of the participants, the interpretation of the semistructured interview was approached by using the following validation strategies: (1) as mentioned by Creswell (2013) bracketing the researcher's opinions by setting them aside and (2) using triangulating methods to ensure that the data is not biased. This included using an external auditor to analyze the data and confirming with the participants that the results are accurate after the data has been analyzed (Creswell, 2013). For a detailed process of the interceding method, please see Table 6 below. 
The reliability of the coding process of the emergent themes was established using the following steps: (1) Intercoding was done by the main researcher and one doctoral student, (2) Intercoding was done on three of the fifteen interviews, which respects the $10 \%$ ratio recommended by Mouter \& Vonk Noordegraaf (2012). Please see table 6 below for the breakdown of the protocol established. In most research where intercoding is used, a percentage index is calculated (see note 3 ). The text evaluator was chosen based on her experience as a researcher and her knowledge of qualitative analysis. She has been involved with Saint Paul University in a research capacity while completing her MA as well as during her doctoral thesis preparation. The first intercoder reliability index was $75 \%$. Using the intercoder protocol developed by Joulain (2015) the intercoder reliability reached 99\%, which is higher than the minimum of 70\% recommended by Lombard et al. (2002). Intercoding which remained dissimilar was submitted to Professor Malette who suggested more comprehensive codes. 


\section{Protocol for assessing the reliability of the initial coding}

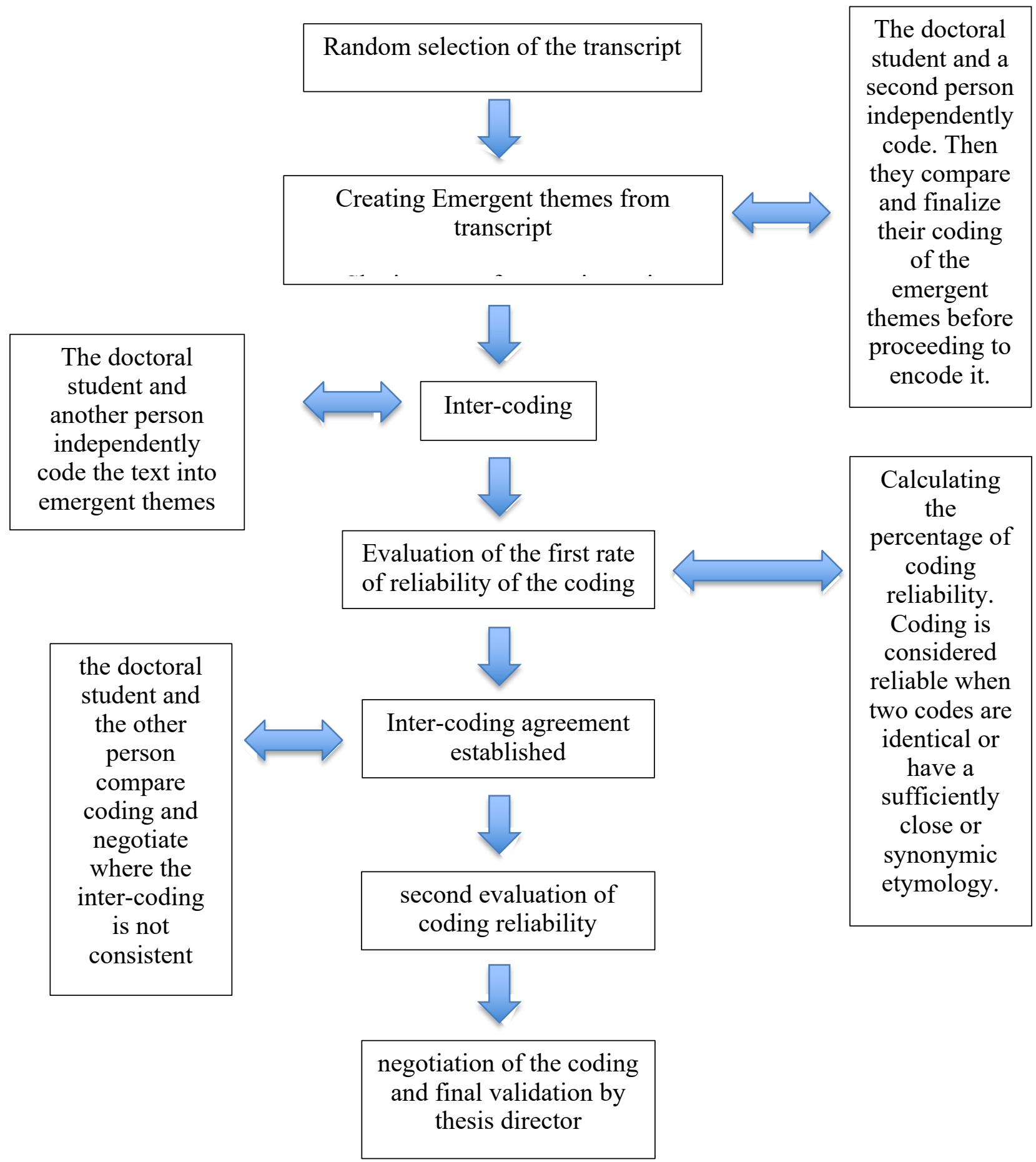

Figure 6: Protocol established from Joulain, S. (2015) who adapted it from Campbell et al. (2013). 


\section{Participant Recruitment}

Six participants were identified according the criteria offered in the literature review through online searches and local advertisements in spiritual based magazines. An email describing the study was sent to each of the participants upon identification who then volunteered to participate. Five out of the six volunteered to be participants in the study. Four of the Participants resided in Ontario, Canada, while one resided in Arizona, U.S.A. Four out of the five had direct cultural origins, while one had been immersed in cultural traditions from a young age. As the study sought to find shamans with a cultural understanding, the inclusion criteria followed what has been identified in the definition of a shaman. Thus, the Participants had to have direct cultural links, experienced the death and rebirth process with direct interaction with spirt guides or spiritual helpers. Although one is active in her community, the remaining four have relocated. Their presence was discovered through word of mouth that created a community of followers in their new locations. Following the acceptance from each participant a consent form was sent explaining confidentiality and the details of the participants' role in the study. Please see Appendix B and Appendix C.

\section{Socio-Demographic Questionnaire}

A socio-demographic questionnaire that covers basic identifying information was administered, with informed consent being taken (Please see Appendix D). This was sent over email to each participant upon acceptance of being part of the study.

\section{Participant Biography}

The study consisted of 5 participants, three males and two females. At the time of the interview Participant 1 was 48years old. He is male, with his primary language being English. He has resided in Ontario, Canada, for the bulk of his life. He is married, has a university diploma and has no religious affiliation. He has strong cultural roots and uses them in his healing work. 
He identified himself as a spiritual person, and identifies a connection with the universe, with the sum of all of his experiences and values humanity. Participant 1 started to have experiences of spontaneously healing people at a young age, as well as engaging in interactions with spirits and a group of spiritual beings later to be revealed to him as the council of Elders.

At the time of the interview Participant 2 was 55 years old. Her primary language is English and is married. She resides in Ontario, Canada. Participant 2 has both a college and university education, identifying strongly with native spirituality. Her experiences began as a child where she had numerous spirit encounters enabling her to see beyond everyday reality. They became her helpers and educated her in the ways of healing. She defines herself as a spiritual person and identifies with the sum of all of her experiences.

At the time of the interview Participant 3 was 53 years old. Her primary language is English, and resides in Ontario, Canada. Participant 3 is divorced, has a university education, identifies as a spiritual person and with the universe. Her experiences began as a child where she was guided by a spirit being whom she later identified with as Mother Mary. Her inquisitive nature led her to seek out Indigenous teachers who guided her in the realm of healing.

At the time of the interview Participant 4 was 67 years old and resided in Arizona, USA. His primary language is English and Spanish, and he also speaks Nahuatl. He identified himself as a single person with a college diploma. He practices no religion, identifies himself as a spiritual person with a connection to the universe, with the sum of all of his experiences and values humanity. His experiences began as a child and was soon recognized by his grandfather, a Nagual, who selected him to be taught in the teachings of his culture.

Finally, at the time of the interview Participant 5 was 50 years old. His primary language is English, and he also speaks Anishinaamebowin/Algonquin for ceremonial purposes. He resides in Ontario, Canada, and is in a common law relationship. He has a college diploma and 
identifies with traditional native spirituality he termed: Midewiwin (Good Hearted Way). He practices his spirituality daily and identifies as a spiritual person. He noted he felt a connection with the universe, with the sum of all of his experiences and identified a direct relationship with mother earth and the spirits of his ancestors. His experiences began as a child where he was in direct communication with his ancestor(s). They guided him and helped him get to a place of understanding within himself, allowing him to impart his teachings onto others.

\section{Validation of Emergent themes}

The emergent themes are reflected in histograms (see p.131 in the Results section for more detail) and represent the data gathered from the interviews. Each emergent theme comprises of meaning units that were found across the Participants interviews. To ensure the data collected had been interpreted as accurately as possible, the researcher randomly chose two

of the five Participants for validation. Participants 2 and 5 were contacted via phone call. During the conversation, the researcher went over each emergent theme and corresponding emerging themes. In each case both Participants contacted validated the information being accurate and capturing the meaning of what they had reported during the interviews.

\section{Challenges to the Study}

Challenges to phenomenological research include: (1) being too structured for some qualitative designs, (2) a philosophical understanding is required to conduct the research and can be too abstract to interpret adequately, (3) the individuals in the study all have to have experienced the phenomenon in question, (4) finding the individuals may be challenging and, (5) bracketing out the researchers experience may be difficult to achieve due to the researcher's own opinion and purpose of conducting the study (Creswell, 2013).

Prior to the study, the researcher had spent several years participating in shamanic activities, such as healing, ingestion of plant medicines and exploring altered states through 
shamanic induction. Therefore, adherence to the protocols offered by Creswell (2013) was initiated alongside careful analysis of the results by the thesis advisory. Since the researcher has had his own ASC experiences, extracting what the description of the shaman's ASC may be biased. This has been accounted for by the selection of the interview process outlined by Seidman (2013). As the interview process works like a funnel beginning with a general understanding to a specific understanding of the ASC, the essence of the interview data will be in its purest form by adhering to the raw data offered by each participant to define what they are saying. As well, the individuals in the study had experience with the ASC, whereas the researcher had limited experiences. This knowledge of experience was determined by the researcher according to the definition created by the literature review on the ASC and the SSC, circumventing the bias the researcher may have had with his own experience. Initially it was thought that finding participants may be challenging. However this changed as internet searchers and local spiritual magazines helped to locate the participants. Finally, bracketing out the researcher's experience was not an issue despite the researcher's personal experience with shamanism. This was accounted for by the researcher adhering to the literature on the subject of shamanism (Creswell, 2013) as well as allowing the interview process to reveal the data.

\section{Ethical Consideration}

Ethical considerations in qualitative research are two-fold: (1) it holds the researcher accountable and responsible for the conduct and validity of the study, (2) while also maintaining the safety of the participants (Creswell, 2013). Due to the personal nature of the interviews, the researcher informed the participants of the purpose of the study, potential risks and benefits of their participation, details of confidentiality, data collection and the storage procedure of the data being collected (Creswell, 2013). Participants were informed prior to the interview that should they experience emotional distress related to their participation in the study, they could withdraw 
at any point during the research process, as well as being recommended to contact a local health care authority. Confidentiality was explained to the participants and to maintain anonymity, aliases would be used in the written thesis and any subsequent publications. Furthermore, participants were assured that their privacy would be maintained throughout the research process (e.g., using pseudonyms such as Participant 1, Participant 2, etc.).

Contact information for the researcher and the researcher's thesis advisor was given if further questions or concerns were required. A signed copy of the consent form was made available to each Participant and the researcher retained a second copy. See Appendix B for the consent form. Lastly, it is important to note this study received approval from the Research Ethics Board of Saint Paul University (see Appendix E). 


\section{CHAPTER VI}

\section{RESULTS OF QUALITATIVE STUDY}

The study incorporated the ideas of Husserl, Heidegger and Sartre by focusing on the shaman's phenomenological experience of the ASC. As indicated in the methods section, a three-stage interview process outlined by Seidman (2013) was utilized to capture the Participant's knowledge of the ASC. The interview process moved from a broad to a specific focus acting like a funnel to capture the essence of what each Participant was saying (Seidman, 2013).

What revealed itself was a general sequence of development that occurred as a result of the ASC experience. Although this was not the study's intention the data offered a sequence that suggested the ASC experiences helped guide, teach and educate the Participants in becoming a shaman/medicine person. Thus, the meaning units were grouped into emergent themes that explain the ASC experience as well as the sequence of development. They are explained below. The reader will recall from the preceding chapter that Creswell (2013) has identified five steps to the phenomenological process. The reader will find a descriptive diagram, figure 4 on page 114 .

\section{Histograms}

Histograms are diagrams showing the frequency distribution which usually use rectangles. In the current study, each histogram presents the frequency of meaning units being mentioned by each of the Participants. Each emergent theme is comprised of corresponding meaning unit reflecting the essence of the emergent theme. For example, the emergent theme 'teachers' is composed of the meaning units: 'animal spirits', 'ancestor spirits' and 'other human beings.' Each Participant is represented by a color and always present in the same order on the histograms. An empty space in a histogram indicates a frequency of zero. For example, in the 
histogram on the emergent theme 'teachers', Participant 2 does not mention 'animal spirits' in the interview.

The emergent themes followed a general theme of evolution that each participant moved through until successfully becoming a shaman. Revealed through the experiences of the ASC, the emergent themes both reflect the experiences and the sequence of development that occurred. They are presented in the following histograms: (1) The term I use to identify myself as, (2) Integration, (3) Learning a New Language, (4) Teachers, (5) Transmission, (6) Application and (7) Archetypal examples of individuation. Each one will now be described in detail.

\section{Emergent Theme: The term I use to identify myself as}

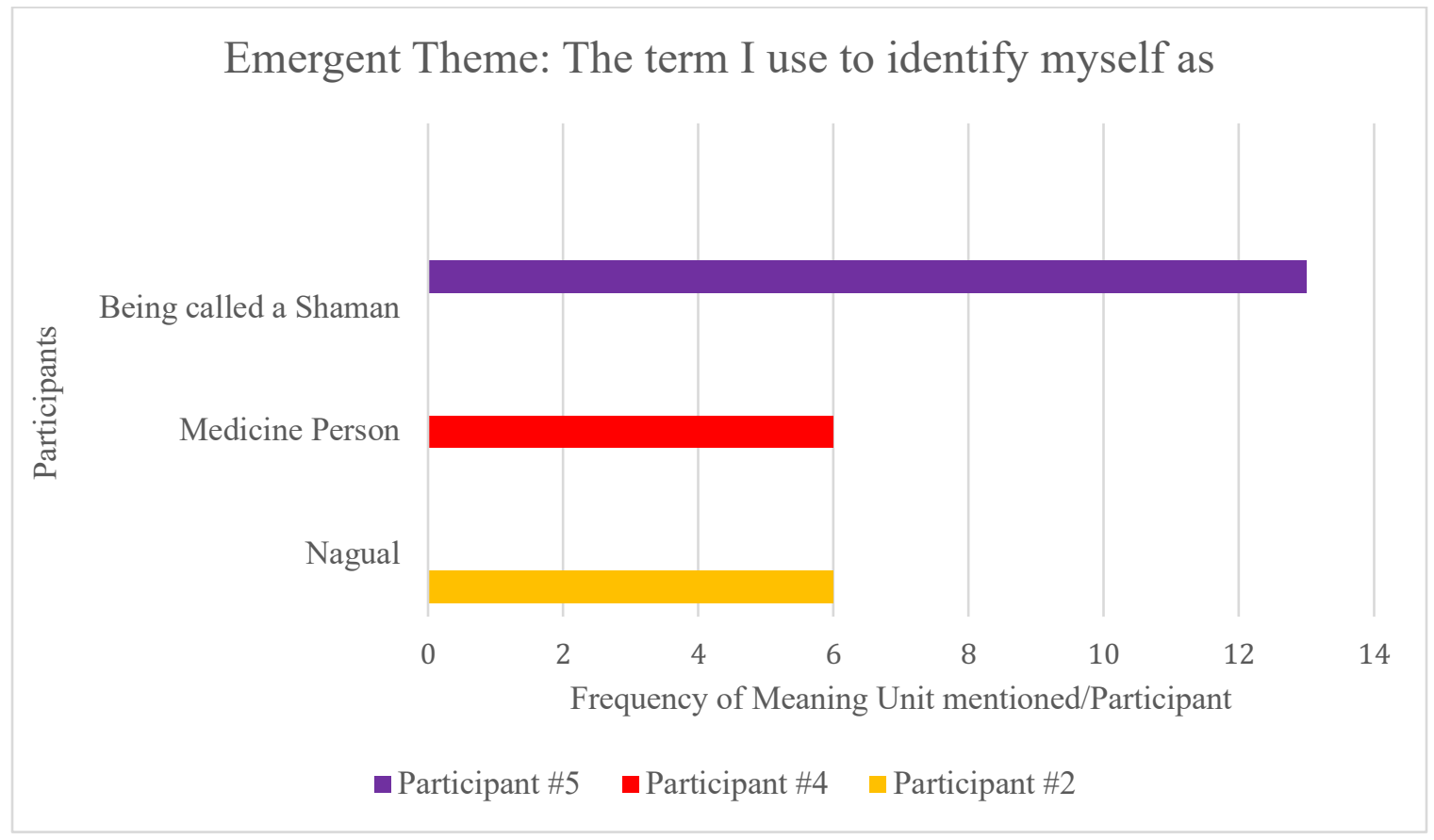

Figure 7. Emergent Theme 'The term I use to identify myself as

This study aimed to identify the specific experiences that each Participant had while immersed in the ASC. Although this was accomplished, exception to the use of the word 
'shaman' during the interview process occurred with the term 'medicine person' being preferred as well as the term 'Nagual'.

The present study identified 3 meaning units corresponding to the emergent theme of 'the term I used to identify myself as.' They include: (1) being called a shaman, (2) medicine person, and (3) Nagual. The phrase 'the term I use to identify myself as' was chosen as an emergent theme due to the definition behind what the word 'shaman' had for the participants. Three out of the five Participants interviewed took exception to the word 'shaman' as it has developed a social meaning in western society. These were Participant 2, Participant 4 and Participant 5. For Participant 2 the phrase 'medicine person' was used instead of the word 'shaman' and for Participant 4 the word 'Nagual' was used in place of the word 'shaman'. For example, Participant 2 emphasized that the phrase 'medicine person' was more appropriate to her. This is due to the traditional teachings she has received, incorporated and developed herself that best describes her understanding of who she is.

Similarly, Participant 4 noted: “First of all, I don't call myself shaman. We don't use that word. We're called medicine people or 'Nagual'. When asked what the difference was he suggested, "spelling... a shaman is a word that came to be... well it's not my word, it's the word you're using, so I'm just telling you it's not the word I call myself, it's your word."

As well, Participant 5 notes:

And I should say that being (called) a shaman and shamanism that's only, that's a belief system. Its old. I think that is why the whole shamanism piece is intriguing for people because at a root level that's how we operate but it's just not part of how we live our life anymore with grocery stores, gas pumps.

The data from the emergent theme "the term I use to identify myself as" has revealed that distinctions occur with terminology. Despite some researchers using the terms shaman and 
medicine man (person) interchangeably (Hulkrantz, 1985), the present study reflects that a difference exists due to the cultural meaning behind them. This will be expanded upon in the Discussion.

Now let us explore the emergent theme 'Integration.'

\section{Emergent Theme: Integration}

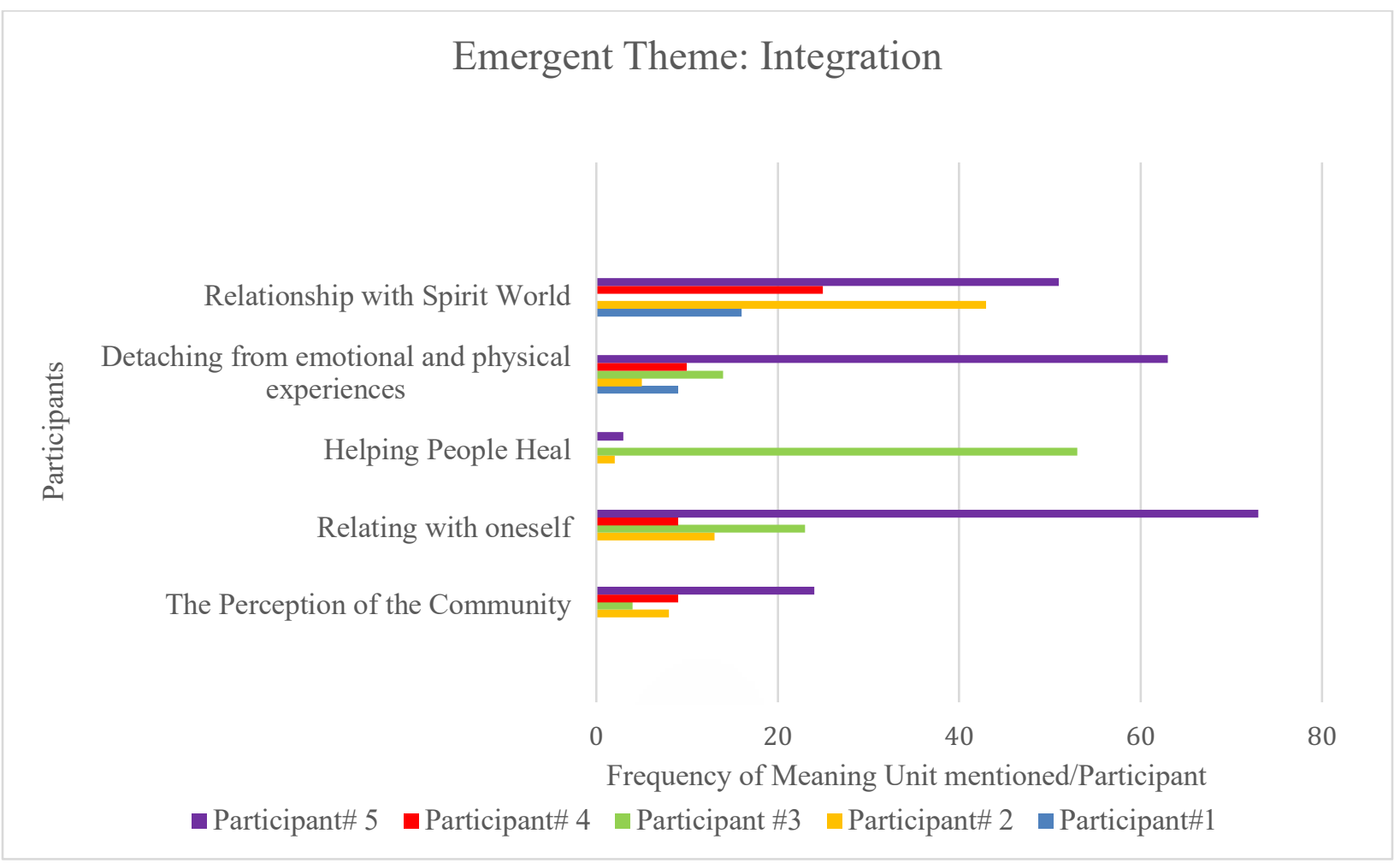

Figure 8. Integration

The word 'integration' was chosen as an emergent theme to capture the essence of how each participant applied the information in the process of becoming a shaman. Thus, to understand the knowledge and stages of development, each participant had to integrate the information accordingly. The present study identified 3 males and 2 females whose narratives suggest common characteristics associated with what a shaman could be. From the interviews, the Participants communicated information obtained from spirits through the ASC that aligns with the literature. 
The present study identified 5 meaning units corresponding to the emergent theme of 'integration'. They comprised of the following: (1) relationship with spirit world, (2) detaching from emotional and physical experiences, (3) helping people heal, (4) relating with oneself, and (5) the perception of the community.

From her experience with the meaning unit 'relationship with spirit world', Participant 2 notes: "it starts with you, with your relationship with you. When you develop that, that is only when you can start developing not only with people but in the spirit world."

For all the Participants, learning how to relate to and to detach from emotional states and navigate and use the ASC as means to gather information for themselves and others became integral to the learning process. To this, Walsh (2007) mentions that guidance comes from inner and outer experiences. Outwardly, this comes from apprenticeship where theory and practice are incorporated through understanding of cosmology, rituals and techniques of culture. Inwardly, the apprentice shaman learns from dreams, visions and spirits. Together the outer and inner worlds eventually align shaping the apprentice into a mature shaman capable of mediating between both words effectively (Walsh, 2007).

Regarding the meaning unit of 'detaching from emotional and physical experiences', Participant 1 notes: "You have to be able to give yourself permission to see and experience whatever's going on in that altered state of consciousness and from there you have to give yourself permission to believe it and that's what changes everything." In his reflections about 'helping people heal', Participant 5 noted:

Yeah, yeah but, but I don't think it's always about making them change what they believe. Yeah because I think that's, that's what we do kind of naturally inside within our own personal spaces. You know it's a perpetual challenging of our belief systems and in what we see what we experience. 
While discussing the meaning unit 'helping people heal', Participant 2 mentioned: "I would have a totally different experience and when you're going into that state you're there to do the healing work so whatever you are feeling I feel whatever you're experiencing, I wouldn't feel that." Participant 3 noted, "But I'm, I'm fully aware that I don't actually heal anybody, and I think any good healer knows that. They're not actually healing a person they're facilitating the experience." On the meaning unit 'relating to oneself', Participant 2 notes:

It's difficult to separate it because I look in the context that we're in the center; the spirit is outside, the emotions are outside, the physical and intellectuals, they are all outside, but that's what revolves around us that makes us who we are how we integrate or how we understand the, the person who we are on the inside.

Reflecting on the meaning unit 'perception of the community', Participant 4 notes:

We (friends) were talking about flying and I told them that I flied all the time and I was flying since I was a baby and I told him that I fly all the time and they looked at me like I was joking, and they could see in my eyes that I wasn't joking. They looked at me and called me a big liar and they suddenly turned on me and I never seen that before. I had never seen anyone turn on me.

Regardless of gender, research identifies similar inner experiences that are common to the initiatory call. These include: messages from spirits through dreams and a vision quests whereby a period is spent in solitude with fasting that allows for a visit by a spirit to occur (Walsh, 2007). Thus, the emergent theme "Integration" revealed that an understanding of oneself is integral to the development of the shaman. The data emphasized that relationship with oneself is vital to this. Corresponding to this relationship is the notion of surrendering to the ASC experience. For example, this led to Participant 1 giving himself permission to believe what is being revealed to him was authentic. This directly challenged the belief systems in place that had 
to be removed in order to accept what was being revealed to each Participant and was eloquently emphasized by Participant 4 who commented on his experiences of flying thinking it was normal for everyone. Finally, the experience of the ASC is different for the shaman as emphasized by Participant 2.

The next emergent theme to explore is 'Learning a new language.' It offers insight into the relationship each participant developed with the ASC, indicating a new language being understood.

\section{Emergent Theme: Learning a New Language}

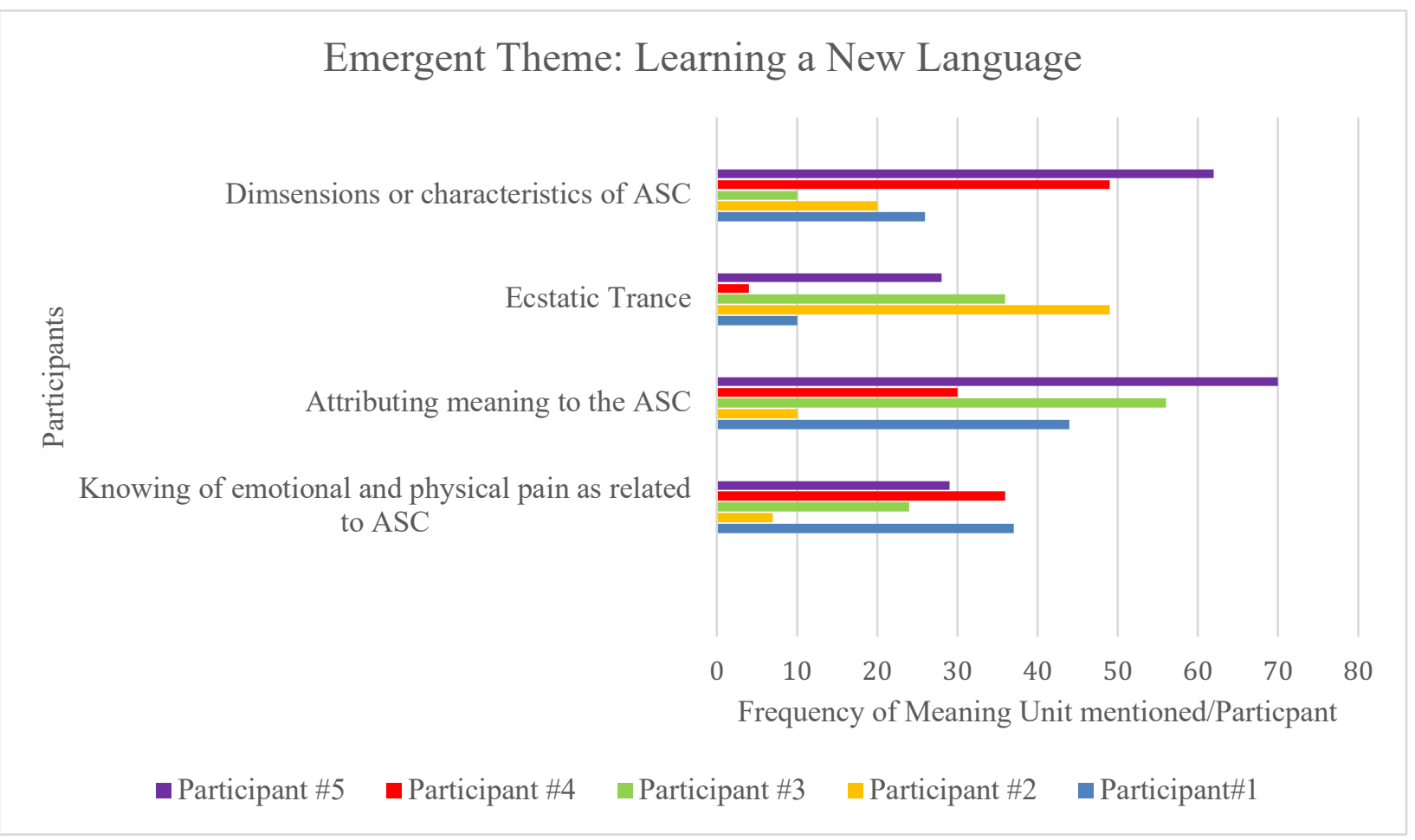

Figure 9. Learning a New Language

The phrase 'learning a new language' was chosen based on the understanding that each participant had to learn new ways of relating to themselves, others and the environment around them while immersed in the ASC. The meaning units included in the emergent theme 'Learning a New Language' are: (1) dimensions or characteristics of ASC, (2) ecstatic trance, (3) 
attributing meaning to the ASC and (4) knowing of emotional and physical pain as related to ASC.

Reflecting on the meaning unit 'dimensions or characteristics of ASC', Participant 1 mentions that,

I will go and be with these council of consciousness beings and I'll sit there and ask them questions because I'm troubled by something and they will literally bring in their perspective. They will always show up, they will always give time.

As well, Participant 4 noted;

It humbles me. Anything that the body can experience you will experience in various states of altered existence. Whatever the body is capable of feeling, you can feel all of those things and probably even a few things that you weren't aware of you could feel to a point where you don't have a body you will be free of it... So all of those things from the imaginable to the unimaginable to the none of the above.

Participant 4 goes on to say: "So I'm kind of in a state of getting guidance or answers through, not so much through my senses but for me it's based on what I'm seeing and sometimes what I am hearing."

As previously mentioned the results of this study support the notion of the ecstatic trance described by Eliade (1951/1980). What follows below are an account by each Participant as indicated in the meaning unit: 'ecstatic trance.'

Participant 1 explains it this way:

Ah, it's awesome! I think that, that, to me schools in! But also, if I need some specific advice I'll go in maybe have a meditation down by the pool and I will go up to the Council of Elders by allowing my consciousness to go to where they are and, and I allow 
him to adjust how I get there by hearing his voice and he guides me in and that's how I get there.

Participant 2 explains: "It's just like when a person comes in through the door...for example when you come in and we sit and talk...Spirits do the same thing, they come in, then we talk.” Participant 3 notes:

Yeah, the times, it feels like there's nothing around me, like there isn't anything. Nothing is three-dimensional. There is nothing and there are voices but they're not like speaking like we would. It's not sonic, its understood maybe, telepathic or something.

Participant 4 explains it in this way:

Everything that I ever imagined was nothing like what it was. It means that suddenly everything that I ever imagined I had put into a nice little box was nothing like what I imagined or wasn't in a little box. All of a sudden you could say that the, the frame of the box disappeared, and nothing was the same as I imagined to be.

Participant 5 describes his first experiences of the ASC describing it as:

I was starting to see flashes of bright light like lightning bolts and I would see them in the evening and in the middle of the day in this house I was living. It was like somebody was outside had this bright camera taking pictures with a very bright flash and that happened for quite a while and I always thought that might be something that was either making me wake up or helping me becoming aware of something beyond me.

On the meaning unit 'attributing meaning to an ASC' Participant 1 had this to say:

"I was seeing things that only somebody observing you from a distance would be able to see and that allowed for me to come out of the fog of whatever these programs are that seem to run most people's lives."

As well, Participant 4 had this to say: 
But if I'm the one having the experience and it seems pretty real and it seems pretty cool and it's made me feel a certain way and made me feel enlightened or empowered or smarter or it's given me an answer about something and its coming from what I'm perceiving as the spiritual direction or the spiritual world and its coming from a Manitou or a spirit or an ancestor or a grandmother or a grandfather or an animal that's representing them.

When asked to reflect on the emerging theme 'knowing of emotional and physical pain related to ASC' Participant 5 mentions that:

And I think what it really boils down to is that it is a real comfort zone for me, that's where I feel most true to myself. I can leave with a real heavy weight on my heart and I can come back completely different because of what I have learned or what I have seen or heard or what I have experienced... with the answers I have been given to help myself or somebody else or somebody's name, like they're spirit name or something.

The data from the emergent theme "Learning a New Language" revealed that an alternate reality exists beyond the waking state where respect is required for communication and the ensuing information being offered. For example, Participant 1 mentioned "allowing" his consciousness to go to the council for information and "allowing" himself to be adjusted. As well, the ASC experience as reflected by Participant 4 resulted in the experience of having no body with "the frame of the box" disappearing, suggesting that his understanding of reality shifted. Finally Participant 5 discussed an understanding of something "making him wake up" into a greater understanding of reality. 
The emergent theme Teachers will now be discussed and is reflected in Figure 10. It offers the reader insight into the how the Participants received their teaching and who the teachers were.

\section{Emergent Theme: Teachers}

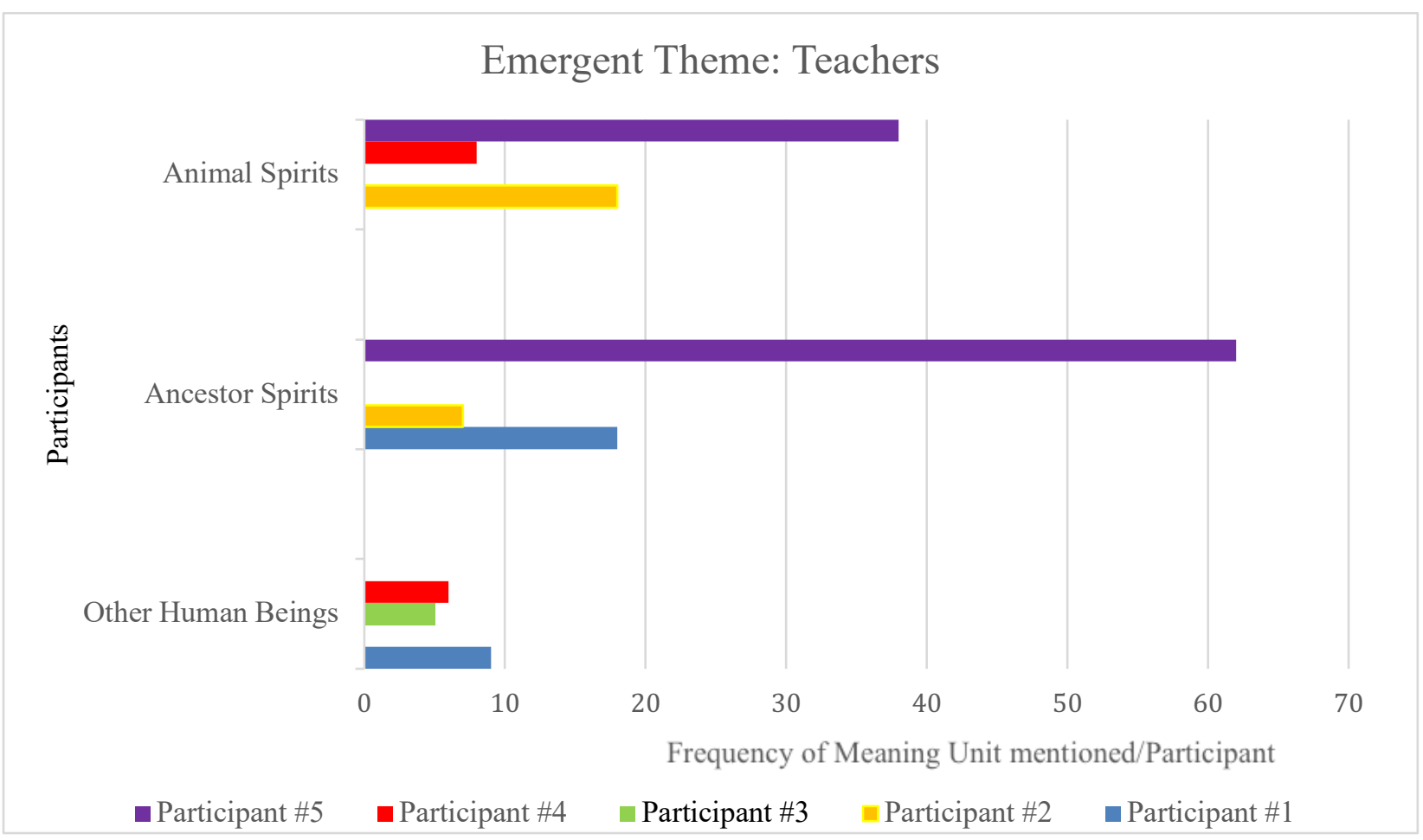

Figure 10. Emergent Theme: Teachers

The word 'teachers' was selected based on the interview data describing how each Participant acquired information related to becoming a shaman.

Thus, it seemed applicable to use the word "teachers" as this is the learning aspect to the process. The data also offered distinctions between the types of teachers and how distinct forms of "teachers" emerged as meaning units. These are: (1) animal spirits, (2) ancestor spirits, and (3) other human beings. Other human beings refer to human beings other than the Participant himself/herself. Both animal and ancestor spirit teachers revealed themselves via the ASC and in the physical world.

For example, an animal spirit is explained by Participant 5 in this way: 
Yah I'm walking on the physical world right on the shell of the earth, but I see something in a certain way, I know based on the knowledge base (of) that animal and I know what that animal means for me at that moment. There's no difference between seeing that real turtle or a turtle form that I am seeing in the clouds. There's no difference. It's the same message it's the same medicine, it's the turtle and it's based on my cultural understanding of what that animal or what that Manitou is, which is also connected to the context of why that answer showed up. So, it's really all the same, there is no separation, that's the way we're taught.

Participant 5 goes on to note that:

The teachers are the helpers because they exist in these different worlds. They're designed perfectly to exist in these certain worlds. So, there are certain creatures that live below the surface of the earth and there are creatures that live on the surface of the earth. There are birds that fly in air above the earth then there are the bigger Manitou's or spirits that exist in the upper realm.

Speaking to what each animal spirit represents, Participant 2 notes that the spirit of the Bear, Thunder Bird, and the Turtle all have specific teachings. Of the bear, she notes:

That's the medicine, okay? That's the one that helps me with the plants. The Thunderbird, that's the one that comes when he sees things all over especially when he is bringing the rains; the turtle is my clan, so I know that one is there all the time, it helps me see okay? Beyond the water, the land, because (that is) the nature of the turtle, so it helps you do those things.

Participant 4 notes that it is also possible to connect with the living spirit of an animal. Of this he explains: 
So, I will give you a little example. When you connect energetically with animal or an insect you will see through their eyes. They communicate not in words but in pictures and visuals... after a while you realize, and you begin to learn, at first you may get fooled at first you may wonder if it's your own mind. But after a while you realize it is not your own mind and they are connecting with you and they are showing you the way they communicate and their interpretation of the world (the teachings) by sending you the energy that makes it possible to see through their eyes... and after a while you learn how to do that and you learn how to navigate that just like you learn how to walk, or ride a bike and it makes it very fascinating. And after that everything changes.

Reflecting on the meaning unit 'ancestor spirits', Participant 5 discusses his interactions with the spirit of his grandmother:

But the more time I spent, or the more time she spent with me and taught me things and was teaching me things. More about plants and things and what to use them for it became apparent that it wasn't my dad's mom. And I really wasn't really sure who it was, but I just felt that she was my grandmother.

Discussing the meaning unit 'other human beings' Participant 1 comments what his physical teacher Jonathan taught him.

(I) came to the... to the realization that I exist in a different, with a different parameter for things that he (Jonathan) struggled with himself which is: knowingness. (Jonathan): explained it that instead of vision, as I don't get too many visions, that one of the highest states of, of uh expression is knowingness and that this is above those who have visions, this is above those who are auditory, this is above those who were even sensory, this is a connection to something that produces instantaneous answers to questions without, um, without the necessities of debate. You get a complete picture, and an answer that so 
complete he couldn't possibly explain it to the person who asked you the question; then I began to realize I'm doing something different. He (Jonathan) gave me a description that this knowingness comes with a tremendous level of experience plus, you know, a connection to the higher self for this great experience over long, long periods of time.

Significant to this development is the understanding of the interconnectedness between human beings and animals (Harner, 1980/1990). Shamanic cultures understood this connection and their myths reflected this with animal characteristics being represented by human behavior such as the coyote being known for its mischievous behavior (Harner, 1980/1990). The data revealed in the emergent theme "Teachers" offered insight into the details of how information was received, interpreted and therefore taught to the Participants. Distinctions between this reality and the next were made while also suggesting of information being present in this reality such as by Participant 5 who noted: "no difference between seeing that real turtle or a turtle form that I am seeing in the clouds." As is being revealed in the previous emergent themes, a sense of respect is paramount to receiving information as stated by Participant 4 who noted communication with animals or insects requires allowing them to communicate their way, not the way we are accustomed to. Finally, the notion of surrender is also apparent in this emergent theme as Participant 1 reflects on receiving "instantaneous answers" to the questions he asks.

The emergent theme: Transmission will now be discussed and is reflected in Figure 11. It offers the reader insight into what the Participants learned in their new roles as shaman and medicine person with the distinction being expanded upon below. 


\section{Emergent Theme: Transmission}

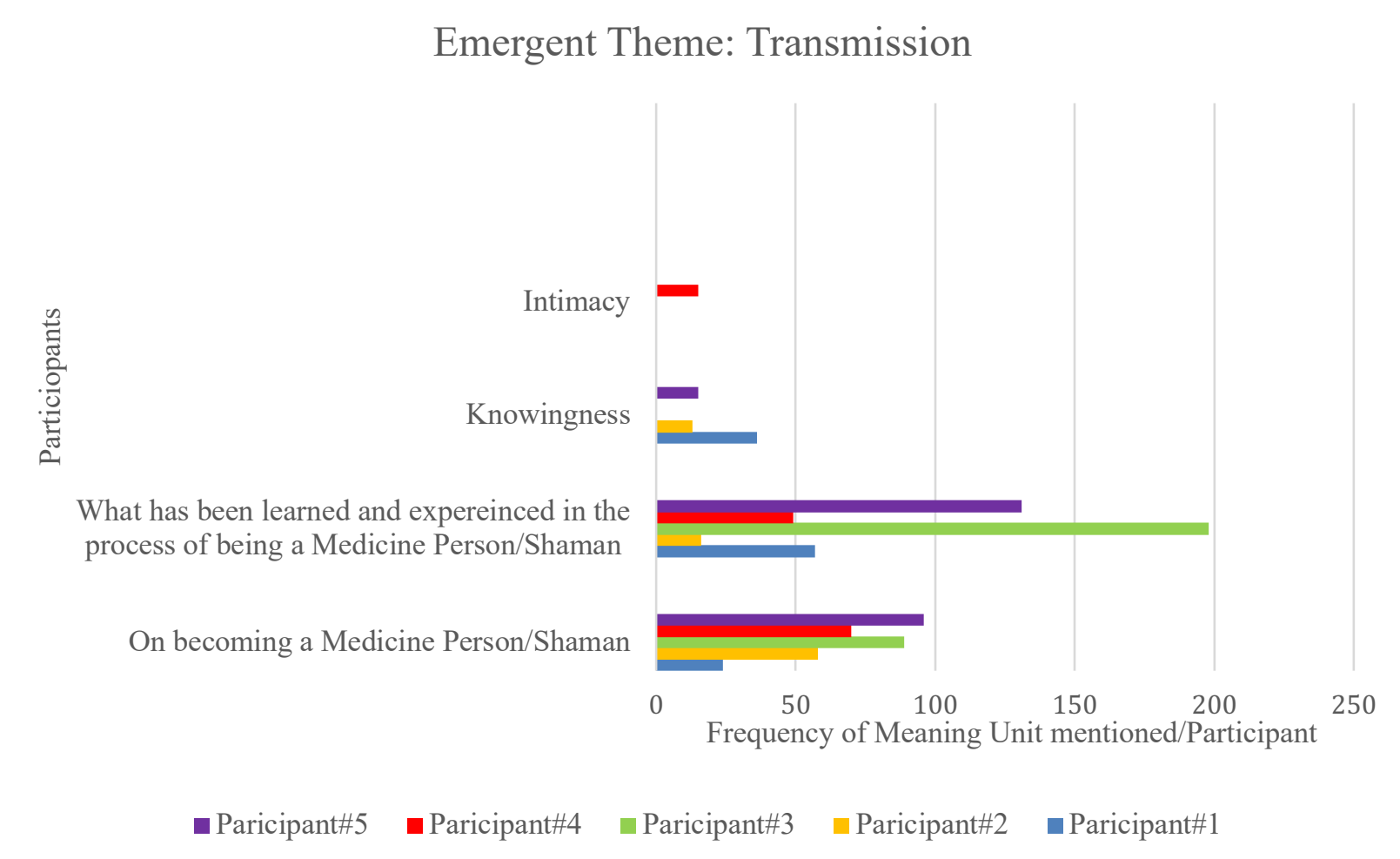

Figure 11. Emergent Theme: Transmission

The word 'transmission' was used as an emergent theme due to the meaning units representing an understanding or communication that was acquired through reflection or direct learning via the experience of the ASC. The meaning units included in the emergent theme 'transmission' are: (1) intimacy, (2) knowingness, (3) what has been learned and experienced in the process of being a medicine person/shaman, and (4) on becoming a medicine person/shaman. The meaning unit 'intimacy' was expressed by Participant 4 and involved his understanding of the internal and external relationship he has developed through his training as a 'Nagual'. He notes:

After a while you learn that you can direct, you can experience in the things that happen to you by becoming more intimate with yourself in knowing how you interact with the world, in how you speak, how you talk, how you think on and on and on and then it starts 
to become fascinating and fun and then you no longer are at the mercy of the world like most people are.

The meaning unit 'knowingness' was expressed by 3 of the participants and involved an inherent understanding of information, how to apply it and how to receive it. For example, Participant 1 notes:

"there was never a need to sort of question it or like I said intellectualize it...as I got older age it just simply was experience and that's all it was. He (teacher) gave me a description that this knowingness comes with a tremendous level of experience so if you come in with the knowingness that can't be tampered with because if you instantaneously feel a shift in vibration.”

Since the use of the words 'shaman' and 'medicine person' have been used by the Participants to distinguish themselves in the interviews, for clarity, a combined meaning unit was developed to identify the different words: "what has been learned and experienced in the process of being a medicine person/shaman'. Despite the distinction that the words had for some of the Participants, the learning process was the same for those Participants who took exception to the word 'shaman' during the interview process. Similarly, a combined meaning unit of 'on becoming a medicine person/shaman was also developed.

On the meaning unit 'what has been learned and experienced in the process of being a medicine person/shaman' Participant 4 noted:

Everything is important even the things I really don't consider important are important. Everything that we, everything that I experience in my life leads to the next thing choice leads to another choice; choice opens up a myriad of other choices, you know, the direction that I move in. It's all the same, it's still happening. I'm still learning. I learned 
that I'm still learning. I learned that I really don't know as much as I think that I do and learned that it keeps changing.

Reflecting more on the meaning unit 'on becoming a medicine person/shaman', Participant 1 noted:

I think the experience for me was to try and explain: what is knowingness?

...Starting off as a child being able to heal people of pain without any prior knowledge of how to do it, just an intuitive sense of how, and being given an opportunity to work on somebody with something as complex paralysis and being able to make them walk in a very short period of time and then I would say running into a higher dimensional being that was able to provide information on a scale you can't get from other humans and that, that was the change right there."

Similarly, Participant 2 noted: "probably started when I was a little girl, (I felt) different than everybody else, not understanding the experiences. Having a pull to plants, nature. Very, very strong pull in not seeing, in the context of things in believing in the context of things that are totally different."

As well, Participant 3 noted:

Each one is expressing their own reality, their perception of what and in fact none of them are true as we all do see the world with these colored lenses and so yeah, it's my job just to empower people basically just empower them. Help them to shed things that aren't serving them like limiting beliefs because your beliefs create your reality.

The data from the emergent theme "Transmission" revealed that an intimacy, as reflected by Participant 4 and a knowingness as reflected by Participant 1 occurs as they became accustomed to and in direct relationship with the spirit world. This allowed for a sense of control over their external environment as mentioned by Participant 1 who noted that he could "direct" 
his experiences. This also corresponds with Participant 4 who acknowledged that the more he immersed himself into the experiences of the ASC his intimacy with life was heightened. He also noted that the more open he became the less he realized he knew. This was further emphasized by Participant 3 who reflected that limiting beliefs help shape our understanding of reality.

The emergent theme Application will now be discussed and is reflected in Figure 12. It offers the reader insight into the Participant's understanding of their roles as shamans and medicine people and their understanding of the ASC experience

\section{Emergent Theme: Application}

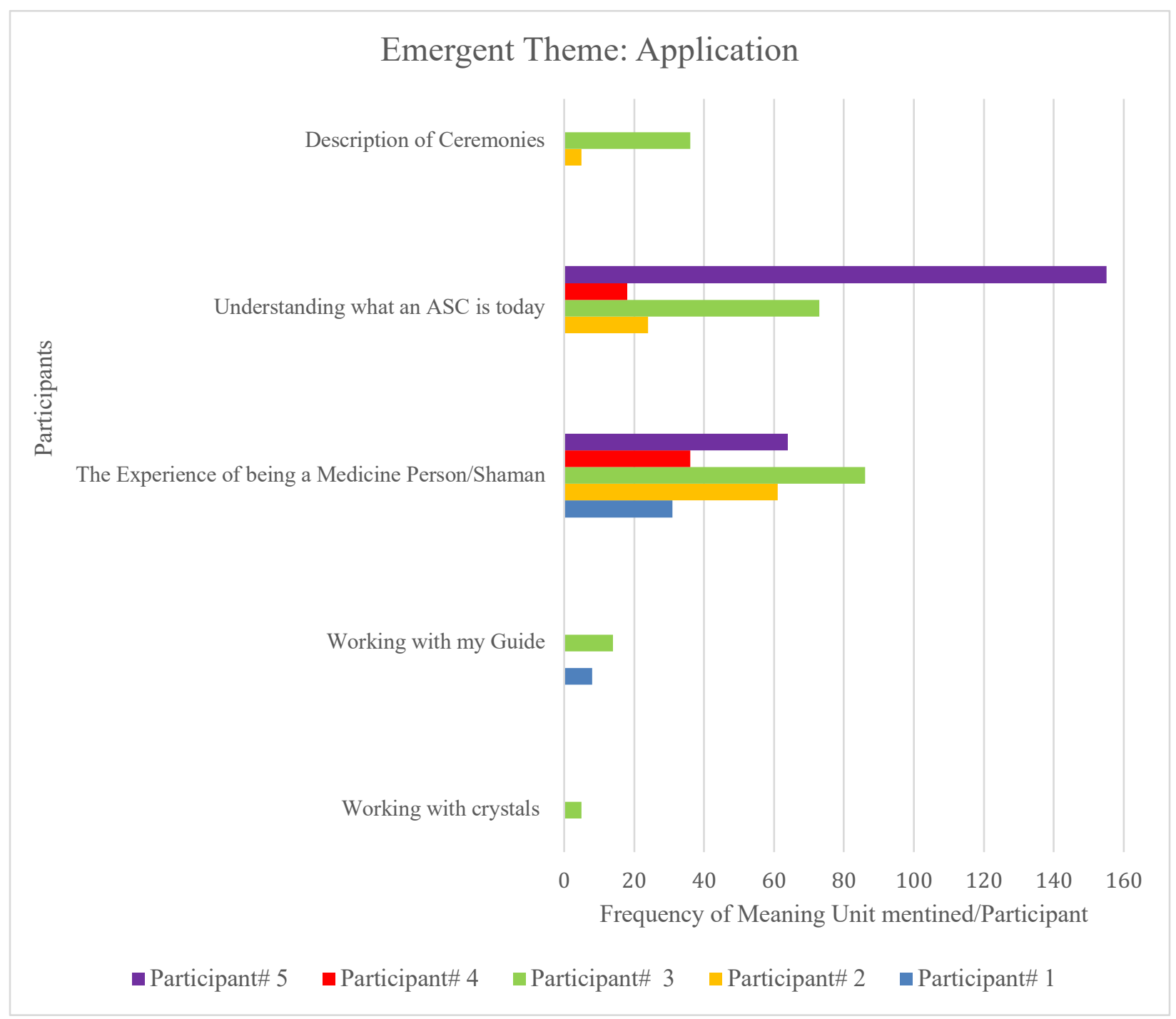

Figure 12. Emergent Theme: Application 
The word 'application' was chosen as an emergent theme due to the process that was common among all participants regarding how the information from their teachers (spirits, ancestors, other human beings) while induced into the ASC has been applied since becoming a shaman. The meaning units included in the emergent theme 'application' are: (1) description of ceremonies, (2) understanding what an ASC is today, (3) the experience of being a medicine person/shaman, working with my guide, (4) working with crystals, (5) relationship with spirit world, (5) being a medicine person. For example, Participant 5 noted on the meaning unit of 'description of ceremonies',

I, I think one thing that might be good to talk about here is um, as an example is the ah, the vision quest so fasting prior to vision question...it's ah making you weaker...making you weaker physically; its making you weaker mentally because you're not eating you're not drinking um so the thinking behind that is that it's, it's making room for that spiritual part of you or that non-ordinary reality part of you to become stronger.

On the meaning unit 'understanding what an ASC is today' Participant 3 noted:

I think at the level of spirit a person is talking about a wholeness of being. Like spirit is all encompassing, like the level of your soul, the level of Creator. So that would include everything you've ever been, you know all of your lifetimes all of your physical incarnations, everything you have thought, said and done and felt, so all of it.

On the meaning unit 'the experience of being a medicine person/shaman' Participant 1 noted, I've grown and advanced based upon interest that I've had in particular topics...each experience would help me graduate from that topic and then move it up the realm to a higher dimension of reasoning about something similar.

On the meaning unit 'the experience of being a medicine person/shaman' Participant 4 noted: 
I learned to walk quietly. I learned to be discerning. I learned that my way is not the way but only a way. I learned that everybody's way is a way, that it is their way. I learned that some people are very adamant about their ways. I learned that we are so adamant about our ways that it is the source of all conflict; it is the source of all conflict that people have within themselves and outside of themselves. It is basis of all relationship.

Participant 2 mentioned that her understanding of 'the experience of being a medicine person/shaman' was:

Having to live in two worlds; knowing that your different; knowing that you outgrow your family. You gotta jump back and forth. First world is the spirit world. spirit world to me was being out in the bush, as soon as I went out fasting I knew I was home, whatever answers I was looking for, the answers would be provided.”

On the meaning unit 'working with my guide' Participant 1 noted:

So, for me they broke me in slowly and allowed me to investigate it so that my curiosity is the thing driving the whole thing. Well I got to know what's behind the curtain! You can't just put a curtain up there because I know what I'll do. I'm just going to go behind it.

On the meaning unit 'working with crystals' Participant 3 reflected:

It's so easy for humans to work with them and I see people come in the store and they go right for the calcite. Already that's like open, easy, for them to do that. And then there are others that are so different you know? Like a meteorite from another planet or something, like not very many people go around that stuff.

The data from the emergent theme "Transmission" offered information obtained from the ASC experience and how it enhanced each of the Participants training. For example Participant 5 reflected on the vision quest ceremony emphasizing that the process opens up the person to 
experience the "spiritual part of you." Participant 3 noted that "a wholeness of being" exists in all humans, and that helping people experience this connection was her goal. As he advanced in his interpretation of the information, Participant 1 reflected that each experience "would help me graduate from that topic and then move it up the realm to a higher dimension of reasoning." To add to this statement, Participant 4 mentioned that he "learned that everybody's way is a way," suggesting that a humbleness has occurred within him as he understood that there is no right way, just experience that leads you. Finally, Participant 2 reflected her challenges with accepting that she had to "live in two worlds" and learned how to pass between them, outgrowing her family along the way.

Let us now look at the emergent theme: 'archetypal examples of individuation.'

\section{Emergent Theme: Archetypal examples of Individuation}

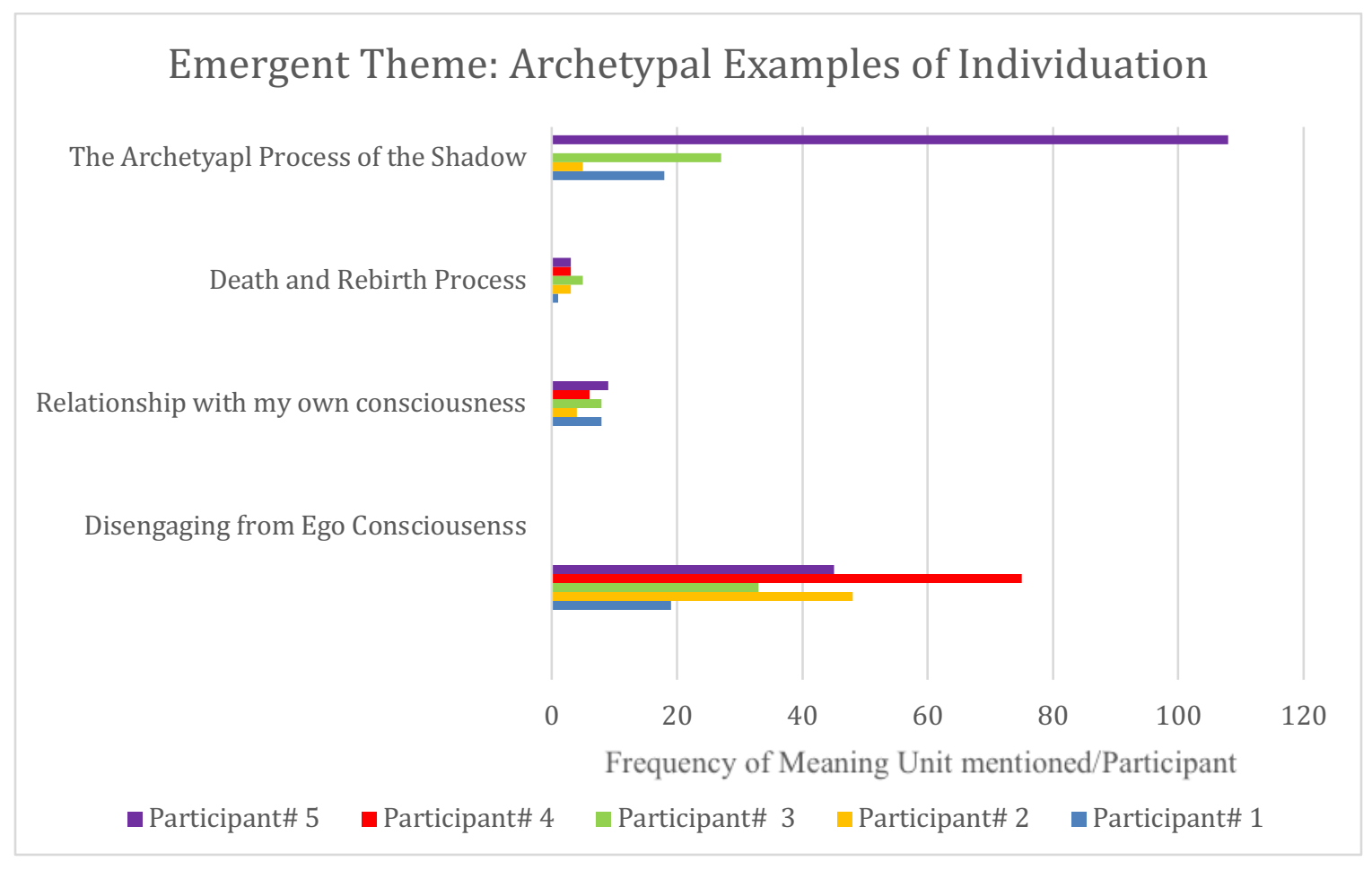

Figure 13. Emergent Theme: Archetypal examples of Individuation 
The emergent theme 'archetypal examples of individuation' was included to identify the distinctions each participant noted in their development. Four meaning units have materialized from the interviews and include: (1) Archetypal process of the shadow, (3) the death and rebirth process, (3) relationship with my consciousness, and (4) disengaging from ego consciousness.

On the meaning unit 'archetypal process of the shadow Participant 3 notes:

"You call it the shadow and it transforms and sometimes those are the strongest parts of yourself because they are the parts that will do the dirty jobs and get you through what you need to survive."

On the meaning unit 'death and rebirth process', Participant 1 mentions:

You could say it was a death and rebirth but for myself it was literally just a transition point it was just a point to say: enough of that, let's start something different and there was no sort of well, no that's not true because now I'm seeing something here. Similarly, on the meaning unit 'death and rebirth process', Participant 2 notes that to become a medicine person you:

Have to go through the bad....Going through the bad is when you're fighting the demons and you actually fighting with the demons... It's almost once you, you past the initiation, once you beat them, once you pass the initiation those things can't bother you no more. And they are not a part of your thoughts, not a part of your being then you know you have the work, the gift of the Creator that you have to do.

On the death and rebirth experience, Participant 3 stated:

I had a near death experience recently where I was just completely out of my body way out beyond these states that we're talking about, and I was with that source, the source energy and when I came back in, I mean things had spontaneously healed, ah everything's changing in my life. 
As well, Participant 4 noted:

That was not my first death, but that was the most powerful of them. First one happened around birth; I would say I had at least half a dozen each has brought me to a different level of understanding.

Finally, Participant 5 mentions:

So yeah there is a lot about that wounded healer stuff, you know how people go through different types of traumas and different emotional experiences that somehow, I guess it is like a death. Yah it's a death and your kind of like reborn. So, whether it's a physical death, that's one thing. It's still a death, it's a change.

The meaning unit 'relationship with my own consciousness' was chosen due to the direct relationship the Participants had with consciousness. Participant 1 noted:

And so we've been able to work an arrangement so spirit acts as intermediary to bring vibratory frequency in to cure the ills of the soul because the soul has had experience on other planets and some of these planets have blown up while we are on it... so if you don't think that that lead to trauma... of course it does.

Specifying that the intellectual aspect of the mind works with spirits, Participant 2 mentions:

"The intellectual, that's the one that works with the spirit and it tells you what you can do...because you're living in the spirit world and you're living in this this world." As well, Participant 3 reflects that:

The expanded state of consciousness takes you somewhere else, you know where you can understand the infinite nature of existence that everything is connected; we are all One and how that is, is actually an ecstatic blissful thing and not something to be feared. Similarly, Participant 4 comments: 
It's sacred. Take that word sacred, expand it. It's the way everything is. Its determined by my relationship towards it ASC is a perception. And the way I look at it is not dependent on how it is but how I am. I will see the world as I am not as it is.

While reflecting on the meaning unit 'relationship with my consciousness' Participant 5 noted: I think I always believed that, but I didn't absolutely believe it and trust it until that moment until I was bigger than I thought I was, that that's what we're made of, we're pure consciousness and another way for me to embrace that is that we are just a spirit. The meaning unit, 'disengaging from ego consciousness' was reflected by Participant 1, who notes, "I learned that I now have access to the biggest Library ever created of all things knowing." Similarly, Participant 2 reflects: "I had to retrain my mind...You have to understand what it's all about... Once you start to understand Ego as a separate entity in you're being then you see it for what it is."As well, Participant 3 mentions:

Because it's like it's getting beyond the ego and the small self that exists in this world and seeing what else is out there and to do that you gotta walk through your shadow.

Participant 4 has also acknowledged this in his interview:

That's where I learned that people do not see the world in the same way that we did; they did not hear it in the same way that we did, they did not participate it in the, the same way that we did, and I learned very, very quickly that the world that I participated in was quite different than the world that I lived in.

Participant 4 also spoke of the difference between a Nagual and an ordinary human:

Let's just say that we have the ability or the capacity to see things that you don't. Because we can see energy. We can see the lines of energy. We can see a person's energy shift, animals, plants. You could say that a Nagual has the ability to see the essence of a person as opposed to just their shell. So that makes life very interesting and very intimate. 
Finally, his first encounter with a Nagual as a child revealed the sense of power they have: ... and as soon as I would think something, he would say it exactly as I was thinking it. I would no sooner think it he would say it out loud and all of a sudden, I realize that he was reading my mind and I had no idea how he was doing it. And finally, I couldn't stand it anymore and I just took off. Took off running. That was my, my first encounter with a Nagual to that extent."

Finally, Participant 5 mentions:

It's like there is no effort because when I get there, it's like physically being there enables me to open up more, even though that is still happening in reality, it is still real. I'm not in my imagination. I'm on the planet, I'm walking on mother Earth, and I'm seeing father sky, and I'm seeing the rock people and the standing people and the trees and all the animals and the things that I experience. You see the way we're taught, there is no separation between the two. It's all perception...it's just another level of awareness, you know. So, for me that is what I embrace the most when I am out in the natural world.

Data from the emergent theme 'Archetypal Examples of Individuation' revealed that the ASC experience allowed for each Participant to experience a transformational process that resulted in going beyond the confines of the ego as noted by Participant 2 who reflected that she had to "retrain" her mind. As well, Participant 3 emphasized a disconnection with ego consciousness. This allows for the medicine person/shaman to experience a greater understanding of reality as revealed by Participant 4 who noted he could see the essence of a person in the form of "energy." Following this Participant 5 noted that his understanding of the ASC allowed him to connect to his understanding of reality through perception. As this was noted in the literature review, the shaman's transformation resembles the individuation process 
(Sandner, 1997/2012; Merchant, 2012) that Jung believed to be an inherent process that all people can go through once the archetypal structures begin to be actualized and made conscious (Jung, 1959a/1980).

This concludes the Results Section. A detailed account of the findings will be outlined below in the Discussion section. The topics for the discussion include: reflections on the research questions, the discussion of figure 14 : the shaman's model of development. The shaman's model of development reflects the model of development as viewed through each emergent theme with exception of "the term I used to identify myself as". This was omitted as it was not considered as part of the developmental course that each Participant took, however is included in the discussion as it was identified in the data. 


\section{CHAPTER VII}

\section{DISCUSSION}

Detailed accounts of the shaman's experiences of the ASC are varied with discrepancies about the quality (phenomenal experiences during the ASC, such as sound, sensations; Cardeña, 2011a) of the ASC (Harner, 1980/1990; Hulkrantz, 1985), what is experienced during it (Noll, 1983) and how it occurs (Eliade, 1964/2004; Walsh, 2007; Winkelman, 2010; Krippner, 2012; Peters \& Price-Williams, 1980), differing amongst researchers. Thus, the description the shaman's experiences from a subjective perspective remains unclear and has yet to be defined/described.

What is consistent amongst researchers is that the shaman does enter the ASC and its uniqueness is reflected in the transformational effect it has on the shaman and others (Harner, 1980/1990; Walsh, 2007). Therefore, this study sought to add clarity and understanding to the shaman's phenomenological experience of the ASC by interviewing five shamans. As well, Walsh's (2007) criteria for determining the experience of the shaman's ASC were used for validity. These have been offered again on p. 155. Walsh's (2007) criteria were chosen as it reflects the shaman's ability to self-heal, suggesting the likelihood that the archetypal process of individuation has occurred. The reader is reminded that Jung's notion of individuation is a process that allows for the shedding of limiting thoughts and beliefs about oneself and the world (Jung, 1959a/1980). This will be referred to throughout the discussion.

The following questions were developed based on the influence of Husserl, Heidegger and Sartre:

1) What are the set of described experiences that the shaman has while induced into the altered state of consciousness?

a) How does the shaman describe his/her experience on a physical level? 
b) How does the shaman describe his/her experience on an emotional level?

c) How does the shaman describe his/her experience on a spiritual level?

d) How does the shaman describe his/her experience on a cognitive level?

The answers to the interview questions were reflected in the emergent themes described in chapter 6. As indicated, each emergent theme had corresponding meaning units that reflected the content of the answers. The semi-structured interview used for each participant interview, reflected upon Seidman's (2013) interview process (see appendix D) and was used to focus each participant in a process that acted like a funnel to get to the essence of each research question. For example, the emergent theme 'integration' highlights the following meaning units: relationship with spirit world, detaching from emotional and physical experiences, helping people heal, relating to oneself, and the perception of the community. The meaning units, relationship with spirit world, helping people heal all are reflected in the research question: How does the shaman describe his/her experience on a spiritual level? As well, the meaning units 'relating to oneself' and 'accepting the mind as part of oneself' are both representative of the research question: How does the shaman describe his/her experience on a cognitive level? The meaning unit: detaching from emotional and physical experiences is reflected in the research question: How does the shaman describe his/her experience on an emotional level? Finally, the meaning unit 'the perception of the community' is reflected in the research question: How does the shaman describe his/her experience on a physical level?

\section{Reflections on the Research Questions}

The results have aligned with the information noted in the literature and revealed evidence suggesting that the shaman/medicine person's role has a greater importance than was once considered. Specifically, the results detailed the experiences of the ASC while also showing that the ASC acts as a tool of development for the growth of the shaman/medicine person. 
Outlined as emergent themes in the Results section the sequence of development presented itself as evidence of the data offering proof for the essence of the phenomenon, the ASC, in the five participants to fully reveal itself. For example, Smith (2007) understands the shaman's development as an illustration of the individuation process of Jung (1959a/1980). As well, the notion that the shaman utilizes concepts that are reflected in quantum physics as reflected by Wolf (1991), highlights the shaman's ability to see phenomena that aren't visible to the general population. The reader is reminded of Participant 4 who reflected his ability to see energy:

Let's just say that we have the ability or the capacity to see things that you don't. Because we can see energy. We can see the lines of energy. We can see a person's energy shift, animals, plants. You could say that a Nagual has the ability to see the essence of a person As well, the results coincide with Walsh's (2007) phenomenological description of the ten dimensions required for defining the shaman's experience of the altered state. These include: (1) degree of control; (2) awareness of the environment; (3) ability to communicate; (4) concentration; (5) degree of energy or arousal, (6) degree of calm; (7) emotion; (8) sense of identity; (9) out of body experience (OBE); and (10) the nature of inner experience.

Furthermore, the Participant's narratives suggest that the definition of what a shaman is requires further development. Indeed, even the use of the word 'shaman' revealed limitation with some participants preferring 'medicine person' or their own word (i.e. Nagual) for what they have become. It has also brought insights into the nature of consciousness and how it is viewed in Western society. As well, it has brought forward a new understanding of the collective unconscious, suggesting that the shaman/medicine person interacts with this to acquire information for the benefit of himself/herself and others. The above suggests that the shaman/medicine person's role and the model of development have changed since its original 
conceptualization by researchers such as Winkelman (2010), who maintains that shamanism is reflected in the culture it originates from.

This study notes that the Participants' narratives suggest that the shaman/medicine person in modern day society has evolved to meet the changing needs of culture. Thus, the emergent themes of the interviews will be interpreted as a new archetypal model of development as revealed through the $\mathrm{ASC}$, of the shaman/medicine person and its relevance for the modern world. They include: (1) Integration, (2) Learning a New Language, (3) Teachers, (4) Transmission and Application, and (6) Archetypal examples of Individuation. Each is to be looked at as a corresponding phase of development that the Participants moved through to achieve their status as medicine person or shaman.

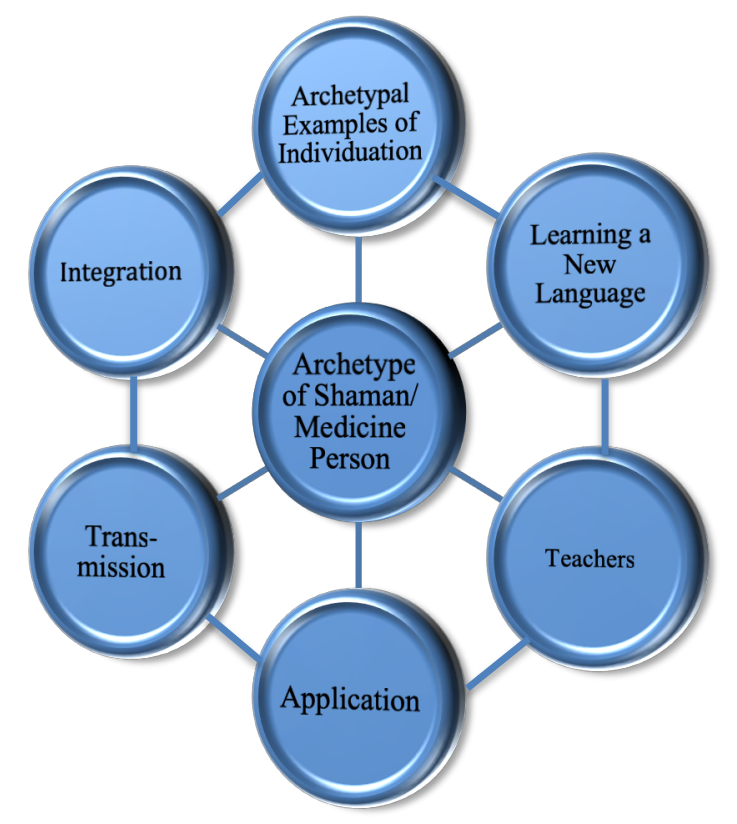

Figure 14. The Shaman's model of Development

The diagram represents the archetypal process of the shaman/medicine person. The connection between each emergent theme indicates the interconnected aspect of becoming a shaman or a medicine person as reflected through the center: the archetype of the 
shaman/medicine person. The center represents the result of the process of archetypal development as well as acknowledging the terms of shaman and medicine person. It is to be considered as the binding force that all emergent themes correspond to, with the top of the diagram reflecting the transformational process as reflected by Jung as the individuation process via the ASC. Thus, the diagram has been created to reflect this with the central understanding of the shaman/medicine person being highlighted as the end result that each participant achieved in their respective archetypal developmental process.

When the diagram is considered from the perspective of how each emergent theme links to one another, an integrative resulting archetypal developmental process is revealed. According to Jung, the developmental process of the individual is mitigated by the Self archetype offering the unique archetypal process for the individual to experience (Stevens, 1994). Crucial to Jung's understanding of the psyche was his awareness of the archetype's beginning. This led him past the development of western thought to humanity's primordial start. This he understood through the archetypes he encountered personally as well as from his dream analysis of his patients. For embedded in the mythology of history are these primordial images that have laid the groundwork for the evolving archetypal process of humanity (Jung, 1961/1990). Thus, as humanity has evolved so too has the archetypal process or the many evolutions the world has seen would not have occurred. The same can be said for modern culture. Both humanity and culture evolve on the micro and macro stage. This also relates to the figure of this thesis, the shaman/medicine person. The narratives have revealed an archetypal process that has been encouraged by the continued interest in the shaman/medicine person, keeping this figure prominent. The adaptation of the shaman/medicine person to our modern times has put forward an archetypal process that allows for the shaman/medicine person to remain current and relevant to our evolving needs. The emergent theme: archetypal examples of individuation reflected Jung's process while the 
corresponding emergent themes of: integration, learning a new language, teachers, application, and transmission revealed a detailed, yet separate, archetypal process. As Stevens (1994) notes, Jung conceptualized the psyche as a self-regulating system upholding equilibrium just as the body does. This suggests that the relationship between conscious and unconscious is compensatory (Stevens, 1994). This process, known as the principle of compensation was central to Jung's understanding of how the psyche adapts and develops through the archetypal process during the course of a life cycle (Stevens, 1994). As the diagram suggests, the person who becomes a shaman/medicine person adapts to the evolving criteria revealed in the emergent themes as a new archetypal developmental process. Thus, the overarching theme is the new archetypal process.

\section{Emergent Theme: The term I use to identify myself as}

Considerable issues have arisen in the literature concerning the use of the word 'shaman' suggesting that scholars decide how terms should be used and when. Based on the information from the Participants' narratives the use of the word 'shaman' limits, distorts and adds a social meaning to an individual who has the ability and knowledge to acquire information willingly with the ASC to help others in need (Craffert, 2011; Hulkrantz, 1985). Despite it being used freely throughout the text, it is evident that the word "shaman" is a point of contention for research and in this study. For example, the term 'medicine person', and 'Nagual' was preferred by two Participants who both acknowledged limitations with the word 'shaman.'

The term medicine-man finds its roots in the Latin medicina, translating into the physician's art as well as the use of curative substances and has kept its meaning due to the primitive nature that the word has. It has been used primarily to describe American Indian doctors, and the word 'shaman' with Siberian doctors (Hulkrantz, 1985). Similarly, and as indicated earlier, Eliade (1951/2004) borrowed the word saman from the Tungus that later 
became popularized into the word 'shaman'. In the case of this study the same word has been used to convey what is being studied, however has also been confused by the sociodemographic nature it was studied under.

In his research, Brinton (1894) mentions that the earliest use of the term 'Nagual' was found in 1530 in Honduras, by the historian Herrera who noted the individual as one who appeared to the indigenous people as an animal, acting as a guardian or companion. Different forms of the word were used, such as naualli by missionaries to New Spain who identified the person as a master of mystic knowledge, one who can use black magic, thus being compared to a wizard or a sorcerer (Britton, 1894).

Hulkrantz (1997) notes that Native American medicine is "strongly traditional" (p. 2). Despite the diversities that occur, medicine work is rooted in the tribe or group the shaman is part of and conform to the rules that have been ascribed to them. Thus, aboriginal medicine belongs to aboriginal people just as western medicine belongs to western people (Hulkrantz, 1997). With that, the difference that scholars ascribe to the word 'shaman' and 'medicine person' is subjective. Hulkrantz (1985) further elaborates on this by stating that distinctions such as these suggest that scholars decide how terms should be used and when. As well, the definition also changes depending on which scholar you are reading. Objections thus have arisen due to the research being done and the observations being made (Sidky, 2010; see chapter 2). Notably the issue between the words 'shaman' and 'medicine person' rests with the notion of whether both experience ecstatic trance and how healing is practiced (Hulkrantz, 1985). Ecstatic trance was first mentioned by Eliade (1951/1980) in describing the unique ability that the shaman has in attaining information (spirit communication, healing) for the betterment of the individual or tribe he or she represented. All of the five participants in this study verified they experienced this phenomenon. The term "ecstatic" has since been expanded by some scholars into the ASC 
utilized by the shaman and has been further researched and studied (Harner, 1980/1990; Winkelman, 2010).

As noted in the Results section, three out of five Participants took exception to the word 'shaman', with the remaining Participants accepting the use of the word, however noting its limitation. This distinction was clearly represented by Participant 4 who preferred the term 'Nagual' to be used while interviewed. Participant 4 also reflected that 'shaman' was the researcher's word, meaning the word that the Western culture used. This has also been verified in chapter 3, What is a shaman?, where Eliade (1951/2004) was noted to have used the word saman to describe his ethnographic study.

Despite research focusing on the ASC experience and it's manipulation by the shaman as a main factor defining the shaman, it does not consider the implications it has for the shamans or those appearing to be one. Thus the Participants in this study reflected this issue, preferring terms that reflected their title. Although the literature emphasizes characteristics of what describes a shaman (ecstatic trance, death and rebirth process, spirit communication) it takes little consideration of the individual who has acquired these characteristics.

What became clear throughout the narratives was that the boundaries of a word hindered the experience of what was being described (Bohm, 1980). However, this did not interfere with the ensuing ASC experience and the developmental process that emerged as a result of the ASC experiences that each Participant had in the process of becoming a shaman/medicine person. Jones's (2006) study into the history of the use of the word 'shaman' in North America suggests that it had been used by anthropologists and government officials as early as the 1850's to describe the practices of American Indians and First Nations people. Therefore, its meaning has changed over time as scholars were influenced by the rise of behaviorism, structuralism, and especially functionalism that influenced psychology, sociology and anthropology (Jones, 2006). 
Specifically, anthropologist Bronislaw Malinowski’s (as cited in Jones, 2006) functionalism and its influence on the psychology of Carl Jung add depth to this issue. Malinowski would note that the shaman's role is central to the society he or she lives in, as it serves the needs of the individuals within it. Thus, culture operated to meet the needs of individuals rather than society as a whole (Jones, 2006). This is reflected in Figure 14 showing that the shaman's development is integral to the surrounding archetypal process that represents each emergent them. Winkelman (2010) suggests that historically, the shaman's role was crucial to the psychological health and well-being of society as well as providing information on where vital food sources were located, keeping the culture alive. This study reflects that the shaman/medicine person has maintained his/her stature and influence with the interest in seeing a shaman/medicine person for guidance and healing being a worldwide occurrence, echoing Malinowski's claim (Jones, 2006) while also supporting the evolving nature of the shaman/medicine person as reflected in Figure 14.

\section{Emergent Theme: Integration}

The emergent theme 'integration' extracted from the Participants' narratives offers insight into how each Participant integrated the learning being offered through contact with spirit guides, nature, animal spirits and teachers that appeared to them. The literature reflects that a key component to the shaman's development is learning how to engage with the spirit world (Winkelman, 2010; Walsh, 2007). All five participants acknowledged this with the meaning unit being described as: relationship with spirit world. Winkelman (1986) notes that the training a shaman goes through includes induction into trance states via social isolation or auditory methods such as repetitive drumming, physical austerities, use of hallucinogens, all of which can cause unconsciousness and/or collapse. The shaman's journey can begin in early adulthood when signs of spirit interaction is apparent, or as a selection at birth to continue family tradition and 
can be chosen by the individual. Those who have been chosen by spirits or otherwise, have traditionally been men however female shamans are not uncommon. For example 4 out of 5 Participants reported early childhood experiences that suggested they were different from their peers. Participant 1 had the ability to heal, Participant 2 was in contact with spirits, Participant 4 could astral travel, and Participant 5 was in contact with an ancestor he later called his grandmother.

Winkelman (2010) mentions the ASC allows the shaman to enter the spirit world with beliefs in spirit being central of the symbolic cultural, personal and social framework of the shaman. This framework of beliefs allows the spirit to be accepted and for healing to occur. He further notes that it is the belief in spirits that creates the symbolic system of norms, values, behaviors that directs people towards a desired social behavior. Thus, when the shaman interacts with spirits, it allows for this desired system of values to be re-incorporated within the individual or group (Winkelman, 2010).

The data of this study not only reflected the existence of the spirit world but also noted that it is part of the existing world. A key distinction made in the study was that the ASC was not required to access it. This contradicts Winkelman (2010) who asserts the ASC's primary purpose is for access to the spirit world. For example, Participant 5 acknowledges that the spirit world is not separate but a part of the existing consciousness. He concluded that it is our perception that makes the distinction of it being somewhere else.

The study does reflect that spirits are available for healing purposes and are the ones doing the healing work through the shaman/medicine person who acts as a bridge for this to occur. Particularly, Participant 2 noted, " you need to be able to see and understand when the spirits come in to do the doctoring...they help out with the doctoring." 
The meaning unit 'helping people heal' is also worth mentioning as the knowledge of healing comes from the spirit world. Craffert (2011) suggests the shaman be looked as a person employing a cultural understanding of control over of the ASC along with social functions that benefit the community. Similarly, Jones (2006) asserts that the shaman be looked at as a person who has at his/her disposal a "healing system paradigm" (p. 22), rather than a set of techniques and practices. The notion of a healing system that occurs across culture endowed to the shaman adds a level of meaning to the term, rather than the reductionist view it has inherited as it has evolved across disciplines of study. It constellates the wounded healer archetype making the shaman/medicine person into a living example of the wounded healer.

\section{Emergent Theme: Learning a New Language}

The interviews suggested that a new language was being learned, one that encompasses an understanding of the ASC experience. These were reflected in the meaning units: dimensions or characteristics of the ASC, ecstatic trance, attributing meaning to the ASC and knowing of emotional and physical pain as related to ASC. Thus, a system of knowledge was being taught to each Participant. Rock and Krippner (2011) note that ritual is the key factor in the development of a group's worldview and mythology. They continue to suggest that ritual in shamanic societies are a "stylized technology" (pg. 666) with the symbols and metaphors of that system encouraging the relief of suffering and healing to occur (Rock \& Krippner, 2011).

This study reflected similarities reported by each Participant in their experience of the ASC that were reflected in the meaning units noted above. As noted by Rock and Krippner (2011) the specific ritual or language being learned by the shaman is representative of this process. However, this study reflected the language being learned by modern day shamans, all who have received teaching on the ASC by human teachers and spirit teachers (please see the section below on the Emergent Theme: Teachers for discussion). The meaning unit 'dimensions or 
characteristics of the ASC' reflect the symbolic understanding and categorical differences of the ASC experienced by the Participants.

Winkelman (2010) notes that the symbolism of the shaman's experience of the ASC play an integral part to help understand outward stimuli, effecting the biological structure of the shaman through personal and cultural influences. Furthermore, of the ten dimensions noted by Walsh (2007) to define the shaman's experience of the ASC, the final point, the nature of the inner experience, is vital for the shaman. Walsh (2007, p. 239) elaborates on this by asking key questions: (1) are the experiences primarily auditory, visual, or somatic? And (2) are the experiences subtle or are they intense and even overwhelming? These questions point to the meaning units of: attributing meaning to the ASC and knowing of emotional and physical pain as related to ASC respectively.

Rock and Krippner's (2011) account of shamanism discusses the soul flight model referring to Eliade's (1989) notion of techniques of ecstasy" (p.5) with other researchers (Harner, 1980/1990; Winkelman, 2010; Walsh, 2007) also discussing this as a key aspect to the shaman's unique ability to acquire information for the well-being of the individual or group being represented. This study reflects this in the meaning unit 'ecstatic trance' noting the variety of emotional experiences outlined by research, such as Walsh (2007) who has reflected upon dread, pleasure, fatigue and feelings of emotional depletion.

\section{Emergent Theme: Teachers}

The information the shaman requires to conduct healing work is integral to the practice of shamanism. This involves an understanding of cultural practice and mythology that is handed down through interactions with teachers (other shamans) and through interaction with spirits (Winkelman, 2010; Walsh, 2007). Winkelman (2010) notes that the shaman's interaction with spirits offers the shaman access to learning through a developed process in the mind that is 
symbolic in nature. Crow Dog, a Sioux medicine man states in his autobiography, "I have no education, but the spirits talk to me. I listen, and I learn about relationship, I learn about spirits" (Crow Dog \& Erodes, 1995; pg. 59). Much like Jung's assertion that the key to understanding our lives rests in the role the archetype plays, the symbolic nature of the spirit is a key factor in the shaman's development (Winkelman, 2010). Furthermore, Winkelman (2010) states that spirits are the most important symbols in shamanism, offering the shaman a system of representation that surpasses physical life. As well, Walsh (2007) and Eliade (1964) both note that through contact with spirits, the shaman establishes a structure to understand, communicate and mediate with spirits what is best for the individual or group of people the shaman represents (Eliade, 1964; Walsh, 2007).

Results from the current study confirm the shaman having access to spirit guides, animal guides and ancestor guides to help them acquire information that is beneficial to helping others (Winkelman, 2010). The Participant's narratives reflect that the spirit guides have an intimate connection with them. For example, Participant 5 notes from his interview that there is no difference from seeing an animal on the land or an animal spirit guide and their presence offers him guidance and answers that he is looking for. This inherent trust also involves surrender. Participant 4 noted his understanding of connecting energetically to an animal or insect requires him to surrender his mind to the animal he is connecting to. This suggests that a deep respect for living beings must be present in order for such an experience to occur.

Teacher spirits guide, coax and educate the apprentice in the unseen terrain where power and knowledge will be attained. This includes an understanding of how to navigate the map of the inner world including understanding how to engage with spirits. It is the spirits who provide information, power and help to the shaman, without them the shaman is at a loss (Harner, 1990; Walsh, 2007). Although the understanding of human/animal interconnectedness is not readily 
seen in our ordinary reality, Harner (1990) notes that during the ASC both shaman and animal communicate. This is seen in the Australian aborigine concept of 'Dream Time' where a parallel word exists alongside ours that is accessible during dreams and visions. It is in this world where the shaman interacts with ancestors, other shamans and animal spirits (Harner, 1990; Ryan, 1999).

\section{Similarities between The Collective Unconscious and the Emergent theme: Teachers}

The data obtained from this study shows strong support for the existence of the collective unconscious. A link can be made between the spirit world of the shaman/medicine person and Jung's concept of the collective unconscious. The study verifies that the shaman/medicine person accesses the ASC and his/her ability to obtain valuable information from beings and figures known as spirit guides and ancestors to help others in need. This shows evidence of the shaman/medicine person's encounter with archetypal figures of the collective unconscious (Jung, 1959a/1980). Sandner and Wong (1997/2012) note that both the shaman and Jung's analytic psychology both identify a separate place that the psyche has access to, with shaman's referring it to as the spirit world and Jung as the collective unconscious that is inhabited by archetypal figures.

Jung (1933/2001) suggests we consider the collective unconscious as a continuous flow of images and figures that occur during sleep and during "abnormal states of mind" (p. 191). Furthermore, Jung (1959a) notes "unmistakable" (p. 5335) connections to mythological ideas, establishing a "species of singular beings" (p.5335), displaying distinct personalities, free from doubt, conflict and suffering. Suggesting that the archetypal forms belonging to a forgotten past and that of the minds of forgotten ancestors and time periods, Jung concludes that their emergence in our lives causes upheaval due to these factors (Jung, 1959a). 
Although many researchers in psychology are reluctant to suggest that the collective unconscious exists, such as Hunt (2012) who argues that the content of the unconscious is unresearchable with the phenomena of it being "more distant and speculative" (p. 80), Grof's research has suggested otherwise. Grof (1985) mentions that material derived from psychedelic research "brings strong support" (p. 190) for the existence of the collective unconscious. Grof concludes that Jungian psychology adds clarity to the perinatal experience such as with the various reports of mythological images and themes that often occur during this stage. As well, Jung's understanding of the nature of the libido, differences between ego and Self, the conclusions made about the role the unconscious has, and the individuation process, can be independently confirmed by his patients and those who had no prior knowledge of Jung (Grof, 1985).

More recently, empirical studies have also brought forward evidence for the existence of the collective unconscious. For example, in their study Rosen et al. (1991) sought to empirically test the strength of the relationship between chosen archetypal symbols and the meaning associated to them. The researchers proposed that the knowledge of a relationship between archetypal symbols and their meanings should resemble abstract semantic memories (knowledge of language, concepts and relationships between ideas) and have little to no autobiographical references. An Archetypal Symbol Inventory (ASI) of forty images was created with the pictures depicting archetypal symbols that were to be associated with their symbolic meaning. If correctly matched, the researchers theorized that subjects should be able to recall the correct meaning used on a cued recall test. The study found that when participants relied on resources available to consciousness they could not recognize the proposed archetypal associations. However, when participants were shown pairs of symbols and meanings to learn a paired-associate recall procedure, improved recall was observed when archetypal symbols were matched with 
corresponding archetypal meanings than those that were not. Rosen et al. (1991) concluded that the symbol and the associated meaning activated prior implicit associations embedded in memory not otherwise available from conscious recall.

In support of this, Sotirova-Kohli et al. (2013) noted that even though the findings of Rosen et al. (1991) may give support to the existence of the collective unconscious and archetypal memory, it may also only reflect the specific linguistic and cultural aspects of the population tested. Thus, to determine that it is not unique to a population, they developed a German language adaptation of the materials Rosen et al. (1991) used, including an adaptation to the ASI that was translated into German. Sotirova-Kohli (2013) hypothesized that, if certain symbols have a universal, archetypal meanings, then "they should be significantly better recalled if they are paired in a memory task with those meanings than if they are paired with other meanings unrelated to the archetypal ones" (p. 542). The authors noted that their study demonstrated cross-cultural evidence to support the association between archetypal symbols and their meanings suggesting that the collective unconscious and the archetypes proposed by Jung have a universal basis.

\section{Emergent Theme: Transmission}

The emergent themes transmission speaks to the evolving role the shaman/medicine person has in consciousness development.

During the transmission phase of development, the word 'knowingness' or the phrase 'it's a knowing' appeared throughout Participant's 1,2 and 5 interviews and was explained as a level of awareness, that when developed, became permanent. Similarly, the use of the word 'intimacy' was offered by Participant 4 whose understanding reflected a level of awareness developing, that also became permanent. This level of knowing or intimacy allowed for access to the greater experience of consciousness via the ASC as if a door was opened to the Participants. 
Kirmayer (1993) reflects that meaning is a relational experience and its conclusions are based on understanding what feelings, sensations and actions an experience evokes. For the Participants of this study, it became clear that a deeply intimate and personal experience occurred to evoke a transformation allowing each of them to experience consciousness in a new way. This intimacy or knowing allowed for a greater expansion of reality to occur for the Participants, allowing access to aspects of consciousness that are not present in our everyday reality.

\section{Emergent theme: Application}

The application phase of development marks the Participant's ability to apply what they have learned. At the core of this phase is the awareness of that a relationship is developing. In the current study, this was expressed with spirit guides, animal guides and ancestor guides, with nature, oneself, and the universe. Kirmayer (1993) notes that meaning evolves through the reciprocal nature of the new information being received and also how it is transmuted or created into the world of the perceiver. The Participants effectively created their consciousness shift by their actions and response to the changes they experienced. Thus, like any relationship, an exchange occurs with new information being considered and then incorporated into the psychology of the individual. Similar to the process of change seen in the patient in psychotherapy, the relationship developing in the Participants suggests that an understanding of the consciousness experience was deepening. The study provides evidence that a breakdown of the perceptual process has occurred with each Participant developing a knowing of, or intimate relationship with themselves and the world.

Despite widespread variability in how psychological theory views relational patterns being formed early in life, most would agree that our past does have influence over our present, thus influencing our perception of reality. Thus, our relationship patterns and our personality may be linked to our past experience of significant others and events that create meaning. This suggests 
that shifts in perception can occur of what happens in our external world as each individual interacts with their surroundings. As mentioned in the literature review (p. 21), the concept of transference outlines this referring to how the influence of significant others impacts our ensuing interpersonal relationships (Andersen \& Baum, 1994) and occurs when our feelings, wishes and assumptions about past relationships are projected into the present moment, whether in a psychotherapy session or in life (Berzoff et al., 2011). Outwardly these perceptions are manifested, and interfere with our impulse control, judgement, disrupting our conscious experience (Berzoff, Flanagan \& Hertz, 2011). Therefore, the relationship we have with consciousness is predicated upon our experience of it. The shaman/medicine person is a person who has transcended this, placing himself as part of and not separate of the experience. This study reflects this shift noting intimate experiences with ego consciousness as outlined by each Participant as well as a shift occurring that allowed for a deeper understanding of life to emerge.

Thus, having achieved an intimate relationship with oneself and the world around them, the shaman/medicine person interacts with it, seeing himself as a manifestation of it, knowing that a deeper consciousness experience is occurring.

\section{Emergent Theme: Archetypal Examples of Individuation}

Sandner and Wong (1997) reflect that Jung uses the shaman as an example in his reflections on the individuation process. As Jung notes:

The ecstasy is often accompanied by a state in which the shaman is 'possessed' by his familiars or guardian spirits. By means of this possession he acquires the mystical organs which in some sort constitute his true and complete spiritual personality. This confirms the psychological inference that may be drawn from shamanic symbolism, namely, that it is a projection of the individuation process. (Jung, 1967/1970, p. 341). 
Further to this Sandner and Wong (1997) note that the shaman is not a manifestation of a particular cultural phenomenon but refers to an archetypal pattern, universal to humanity, prepared to emerge once the individual is ready, with the archetypal pattern of the shaman results in the individuation process.

As well, the emergent theme 'archetypal examples of individuation' also verified the death and rebirth experience as being an integral aspect to the shaman's/medicine person's development. The results of the thesis indicate that the shaman/medicine person may go through more than one death and rebirth experience. For example, Participant 4 discusses going through "many" deaths. As well, the Participants narrative reflected that the knowledge and awareness of being a shaman or medicine person differed in each Participant. This suggests that an evolution may occur with respect to the shaman/medicine person's development. For example, Participant 1 and 4 both reflected a wisdom that was still developing in the remaining Participants.

Smith (2007) notes that the individuation process is Jung's comparison to the path to the heart whereby the ego-consciousness becomes secondary and in services of the self. The literature reflects that the shaman moves through a death and rebirth process, suggesting that a transformation of consciousness occurs, with an expanded awareness coming forward. Being separate from the ego, this relates to Jung's process of individuation. Smith further elaborates that shamanism reflects this as the shaman is able to be in service to the needs of others by living a life that reflects an understanding of servitude (Smith, 2007). For a more detailed description, please see Chapter 2.

Given that the shaman/medicine person has been through the death and rebirth process, this suggests that the shaman/medicine person has experienced the "wearisome" details of coming to terms with the unconscious aspect of the personality. This allows for the understanding that a connection exists between the shaman/medicine person and consciousness. As Jung notes about 
individuation: "It is a relatively rare occurrence, which is experienced only by those who have gone through the wearisome but, if the unconscious is to be integrated, indispensable business of coming to terms with the unconscious components of the personality." (Jung, 1954, p. 223)

The discussion will now focus on clinical observations made by Stanislov Grof and supporting research as it is an integral piece to the shaman's transformation as depicted in Jung's individuation process.

\section{Clinical Observations in the Death and Rebirth Process}

Detailed clinical observations of the death and rebirth process during LSD assisted psychotherapy by Stanislov Grof will follow. Despite the controversy that using LSD in psychotherapy brings, Yensen and Dryer (1996) note that Grof's theoretical contributions have been firmly situated by the meticulous observation and scholarly descriptions of thousands of clinical experiences by patients undergoing LSD assisted psychotherapy. It is also important to note that current research in this area is being conducted by the organization known as MAPS (Multidisciplinary Association for Psychedelic Studies) where current clinical trials on LSD assisted psychotherapy are being done, alongside other psychedelic compounds such as ibogaine treatment for addiction, Ayahuasca-assisted therapy for PTSD and addiction and medical marijuana for PTSD (www.maps.org).

Winkelman (2010) mentions that the death and rebirth process is a symbolic death of the old self that allows for a new, "higher order self" (p. 109) to emerge. Similarly, Walsh (2007) reflects that this process is common throughout various spiritual disciplines, such as in mystical and yogic traditions. He notes that spiritual practitioners must be open to facing physical death and ego death. Ego death is the end of an old, programed identity and in aboriginal practices concerning important life transitions, rites of passage are enacted as death-rebirth rituals. Walsh (2007) notes that specific spiritual practices are designed to evoke unique experiences. These can 
be valuable, insightful, cathartic and healing to the individual with little differences existing between the spiritual experiences of shamans or others. He cautions that mistakes are made when we jump from making statements about experiences to making assumptions about the reality these experiences reveal (Walsh, 2009).

Grof's work with psychedelics began in 1956 in Prague with an interdisciplinary team doing comparative studies of hallucinogenic drugs, working with diagnoses ranging from depressive disorders, psychoneuroses, psychosomatic disease and character disorders to psychoses. Grof also worked at the Prague Psychiatric Research Institute in the Department for the Study of Psychogenic Disorders from 1960-1967 (Yensen \& Dryer, 1996). From this anecdotal evidence, Grof outlined four basic stages of consciousness that he observed his patients moving through: (1) the sensory barrier, (2) the individual unconscious, (3) the level of birth and death, and (4) the transpersonal domain (Grof, 1985).

Particularly, Grof's emphasis on memories derived from LSD assisted psychotherapy sessions has brought forward claims about the origins of trauma that he observed during his LSD assisted psychotherapy sessions (Grof, 1985). As well, this brings forward subjective evidence supporting the death and rebirth process as a transformational process of the psyche (Grof, 1985). Otto Rank also focused on birth, noting that all human mental life originates in the anxiety of the birth trauma with central human conflicts resting with the desire to return to the womb. Thus, during life any change from pleasure to unpleasant situations results in the desire to re-establish security (Grof, 1985). Similarly, Attachment Theory proposes a need to seek safety, where the person, upon experiences of distrust in their immediate environment, makes deliberate attempts to establish homeostasis (Mikulincer \& Shaver, 2007). Grof's work suggest that this is a process that, once transgressed, opens up the individual to move into the transpersonal domain that correspond to the mystical experience outlined in the literature review. 
Grof's observations of the birth trauma occurred during what he refers to as the Basic Perinatal Matrices (BPM) that appear at the perinatal level of consciousness, defined as: occurring in, concerned with, or being in the period around the time of birth (www.meriamwebster.com). Grof (1985) noted that his patients reported encompassing psychological experience of birth, aging, disease, dying and death). Consisting of four stages the resulting characteristics include: (1) the BPM I stage with reported memories of the intra-uterine experiences to that of cosmic unity on the spiritual/philosophical dimension; (2) the BPM II stage coinciding with the first stage of clinical delivery, including memories of the impact that uterine contractions had on the person before the cervix is dilated with corresponding spiritual/philosophical experiences of having "no exit" (p. 10) or subjective references to what experiencing "hell" (p. 10) is like (3) the BPM III stage describes memories of birth including passage through the birth canal and to experiences of the death-rebirth struggle on the spiritual/philosophical domain, and (4) the BPM IV stage that relates to memories associated with separation from the mother's body on the biological level and to experiences of ego death and rebirth on the spiritual/philosophical level (Yensen \& Dryer, 1998).

Grof notes that once the subjects have experienced the death and rebirth process that involves detailed imagery of the birth process a "profound" (Grof, 1985 p. 100) transformation in personality occurs. Similar to the shaman's experience noted in the literature, Grof (1985) confirms patients reported experiences with visions of blinding white or golden light, being left with the feeling of an immense decompression and expansion of space. This follows an existential crisis whereby the individual concludes that he will have to leave this world to which he has become emotionally attached and that the beginning of life resembles the end of life. Reflective of the emerging theme 'detaching from emotional and physical experiences' from this study, this follows an opening into significant spiritual and religious experiences that form a 
fundamental part of the psyche, independent of cultural, societal and religious backgrounds

(Grof, 1985). More importantly this also suggests that once the death and rebirth process has occurred, that the shaman begins his/her individuation process.

\section{The Wounded Healer Archetype}

The data from the interviews offers evidence in support of the shaman/medicine person being a wounded healer. Each Participant has outlined this process of change that the literature specifies (Harner, 1980/1990; Winkelman, 2010; Walsh, 2007) as the acknowledgement of the shaman's metamorphosis. Integral to the shaman's etymology (Sandner and Wong, 1997) Groseback (1975) concludes that it is the fundamental archetypal pattern required for healing to occur on a deep level. This is reflected in Figure 14 in the emergent theme of 'archetypal examples of individuation' through the 'meaning unit death and rebirth process.' To become a wounded healer, the individual has to incorporate the subjective meaning of his/her wounds to be able to impart it onto another. This allows for a shift in consciousness to occur. Thus, the shaman/medicine person acts as a consciousness explorer and teacher to help others become conscious themselves. Recalling Walker's use of symmetry (2008) and Jung's (1960) understanding of symmetry (see p. 48, Chapter 3 ) the wounded healer archetype acts as the organizing principle behind the shaman's methodology. When constellated, the wounded healer archetype acts as its own system of healing to the individual possessing its characteristics.

\section{Induction Process}

Research has identified induction procedures as the method used to enter the ASC (Cardeña, 2011; Winkelman, 2010). These include ingestion of mind-altering substances, rhythmic drumming, chanting, breathing techniques, fast paced movement until exhaustion, fasting and others (Winkelman, 2010). One induction that was discussed in the interviews and not observed in the literature was the experience of nature. Three of the participants identified 
nature as the place where they received information, help and guidance. The act of leaving home and moving into natural surroundings allowed for a gradual and effective emersion into the natural world. Once separated from everyday life, it allowed for access to information that was needed.

The interviews suggested the ASC is part of the overall conscious experience and is accessible without any specific induction procedure, despite some of the participants discussing meditation and fasting methods to clear their minds. Once this awareness develops, described above and in the interviews as the term "knowingness" or "intimacy" it allows for access when required. Eliade (1964/2004) notes that this becomes a permanent condition, where it is possible to "see" the spirits. Thus, the shaman is a person who leads by example, displaying to the world that it is possible to access the ASC or expanded sense of consciousness at will.

\section{Findings on the Consciousness Experience}

Psychologist and educator Glenn Aparicio Parry (2006) who advocates for a synergistic, interconnected curriculum focused on indigenous knowledge, notes that the main difference between Native and Western science is that Native science describes observed reality and unobserved reality, equally. Reflected in Native languages, this understanding has influenced how reality was structured. For example, the Hopi language identifies two cosmic forms to be inherent to reality, manifested and manifesting. As well, verbs in Hopi language do not distinguish tense as Indo-European structure does. Thus, the manifested world is made up of everything, mental and physical, with no distinction between past and present with a future that is always manifesting (Parry, 2006).

From his research, Walsh (2007) suggests that the shaman's worlds are interconnected with one another, while Harner (1980/1990) mentions that the shaman uses his/her mind to transcend the limits of this reality and move into another. In the literature, it has been reported 
that a shaman is aware of three distinct "worlds" known as the lower, middle and upper worlds where ancestors and spirits inhabit. The shaman travels to them for help and guidance to gather necessary information for himself and others (Eliade, 1964/2004).

With exception to Participant 5, who clarified nine worlds existing, rather than three, the remaining Participants offered information suggesting that the spirit world is just as accessible as waking reality is. The nine worlds acknowledged by Participant 5 included: four worlds, an upper, a middle, a lower and a sky world, as well as mental, physical, spiritual. The remaining two are above the head and below the feet. Research has noted that shamans go to the spirit world to gain insight, acquire information for the healing and benefit of others. This would confirm Harner's (1980/1990) assertion that the main attribute of a shaman is the contact with a "hidden, normal reality" (pg. 17). Winkelman (2010) identifies that the role of the spirit world is for healing and are "symbolic representations" (p. 150), while Walsh (2007) discusses the implications of accepting it as fact, while also acknowledging that the shaman does experience something unique.

It became clear as the interviews progressed that the phenomenological experiences of the Participants were addressing a much larger problem that has plagued research since it began to investigate the consciousness experience: the subject/object dichotomy. All the Participants noted that a connection exists between the spirit world and the normal waking world we are used to. Due to our perception being focused on the structure of this reality, we do not consider that other realities may exist, or one complete consciousness experience. The data from this study identified contact with spirits, including interactions with spirits occurring during the interviews.

In his impressive synthesis of consciousness research, Wilber (1977/1993) argues that philosophy, psychology and science have maintained a theoretical outlook that separates us from our subjective experience of reality. This has altered our view and caused Western society to 
exist in its own altered state. Kuhn (1962) discusses this from his observations of scientific revolutions whereby versions of reality have been altered by our acceptance of belief systems. Finally, Whitehead (2011) suggests that society has made alterations to consciousness for systems of social order and governance to occur. This may have helped structure our experience however it has also altered our understanding of consciousness. The subject/object dichotomy keeps everything in subjective reality separate, focusing on the 'space' between subject and object. This separation has occurred across all levels of thought and perception altering ideas of race, social status and more. This alteration is what Bohm (1980) and Grof (2009) refer to as the Newtonian Cartesian order of thought.

Bohm (1980) indicates that the notion of order being permanent and unchangeable limited our perception of consciousness, while challenging this allows for new ideas, measures and structures to be considered. As well, Grof (2009) argues that his research with psychedelics challenges the prevailing notion of the Newtonian Cartesian view of object reality. Subjects reported 'experiential identification' (p. 447) with other people, animals and nature suggesting that subject and object are not separate rather there is a natural union with all things.

The current study shows that the Participants all report a 'hidden reality' that is accessible at will, challenging the notion that the ASC is required to access it. Thus, the Participants suggest that it is alterations in our perception, an ASC in itself, that have caused variations in our everyday conscious experience that has kept us from what Bohm (1980) refers to as the implicate order, a continuous unfolding process where life changes form eventually becoming life again. It is an understanding that has been known in Eastern traditions of thought for centuries. This unfolding process occurs in everyday life, and it is our perception that alters it (Wolf, 2001). This is easily observed in nature with matter being in a continuous flow of decomposing and composing itself anew. The seed that falls does not become the plant, rather the plant manifests 
by everything that surrounds it: the soil, the sunlight, the air, the rain, therefore is dependent and part of the experience (Bohm, 1985). It is our perceived independence from this that has caused our perception to be altered from the implicate order Bohm (1985) refers to.

As mentioned previously, the Western culture is a prime example of the monophasic state, valuing waking consciousness as the true experience (Winkelman, 2010). Anything altered from this state is considered different or abnormal such as the SRE, TE, ME and psychopathological states (Cardeña, 2011a, 2011b; Grof, 1985). On the other hand, polyphasic cultural worldviews consider the ASC experience as equally important as non-ASC experiences. Therefore, these cultures have incorporated the ASC as a tool for exploration with the individual and cultural identity being informed by the experiences of the ASC (Laughlin et al., 1990). This offers a well-informed understanding of how the consciousness experience unfolds.

When two cultures collide, theoretical, social, and psychological issues result. Craffert (2011) notes that the ASC assumes that a normal conscious or baseline state exists, whereas research has ignored that many conscious states exist (Tart, 2011). As well, fluctuations in consciousness occur all the time (Cardeña, 2011a). Research agrees that consciousness is culturally defined (Whitehead, 2011), thus baseline states from culture to culture are also defined differently. Furthermore, ASC's are not only the result of biological and neurological structures as Winkelman (2010) argues, they are also culturally and societal in origin. The intricacy and multidimensional aspects to them are best understood through the cultural context in which they are informed (Craffert, 2011). Thus, the shaman is best understood through the application and use of the ASC in cultural and social settings, offering a theoretical approach to clarify the biopsychosocial implications of its benefits (Craffert, 2011).

The intention of the shaman is to remove his/her client from their own version of reality and connect to the greater truth that exists, allowing one to unite with it, make conclusions, and 
to learn from it (Harner, 1980/1990). In his book, The Master and His Emissary: The Divided Brain and the Making of the Western Word, McGilcrist (2010) suggests that our left and right hemispheres of the brain have influenced the construction of our conscious experience. This implies that a logically structured reality, resembling a left hemisphere focus, constitutes for a similar conscious experience. Similarly, those that are more attuned to the right hemisphere, have a broader and more fluid understanding of consciousness, such as polyphasic cultures that a shaman comes from. Harner (1980/1990) noted that two aspects of reality exist, ordinary reality and non-ordinary reality. To apply McGilchrist's (2010) argument, both realities that Harner (1980/1990) speaks of exist simultaneously, despite what is observed. Thus, if we hold the belief that the table across from us is separate, it will remain to be so. The illusion lies in how the experience is considered. When we observe, we may notice things as separate but do not consider that we are part of the experience as well. Thus, it is not that the ASC is separate from our conscious experience, the error lies in our perception of reality as having to resemble what we have structured it to be. What research has overlooked is that the consciousness of society has evolved in large part due to people like the shaman, again placing the shaman as an individual whose role is adapting to the growing needs of the world.

\section{Limitations to Research}

The main limitations to research are the sample size and the method used. Qualitative methods of inquiry offer subjective accounts of what is being studied. Thus, quantifying this data becomes challenging as it is difficult to measure accurately. However, the subjective experience is how we perceive our reality, bringing into question the limitations of research designs. Of all the challenging subjects in research, consciousness has been one of the most difficult to describe. After all, it is the human being who observes, defines and creates stories about subjective experience. 
Although the participants received information during the ASC experience some also received information from other human beings that also was considered part of their development. Ryan (1999) notes that the shaman's training is multifaceted and as the shaman develops the ability to access deeper levels of consciousness via the ASC, the shaman learns how to apply the teachings accordingly. Distinctions begin to emerge between ego and self, giving the shaman the ability to integrate ego disillusionment so to navigate the unseen world appropriately (Walsh, 2007; Ryan, 1999; Winkelman, 2010). Similarly, Jung outlines this in the unfolding process of individuation where the individual comes to an understanding of what the archetypes are (Jung, 1959a/1980).

\section{Clinical Applications}

The use of shamanic approaches in psychotherapy is not a mainstream affair. Due to ethics and the limits of what psychotherapy can achieve in a typical therapeutic hour, incorporating techniques associated with shamanic work is challenging. However, the process that the shaman/medicine person engages in with his/her client is similar to that of psychotherapy. Psychotherapeutic approaches aim at uncovering unconscious material rendering them conscious to the client. This allows for integration, understanding and reflection to occur. It is the engagement with the spirit world or the collective unconscious where understanding occurs. Working from this foundation may thus impart changes similar to that of the shaman/medicine person. The Foundation for Shamanic Studies offers intensive training on shamanic ritual, technique and process. A five-day course is offered on Harner Shamanic Counselling ${ }^{\mathrm{TM}}$ that offers an approach towards personal problem solving and guidance for those who chose to use the methods professionally (www.shamanism.org). Another possibility would be to incorporate the shamanic journey into client sessions to help the client move into unconscious content. Before this can be achieved the practitioner would have to consult with the 
respective college before this is offered. This can also be learned from the Foundation for Shamanic Studies as well as other shaman training programs that are offered to the public. Finally, the University of Minnesota offers a course in The Foundations of Shamanism and Shamanic Healing (https://www.csh.umn.edu/node/1476). 


\section{REFERENCES}

Aghajanian, G. K., \& Marek, G. J. (1999). Serotonin and hallucinogens.

Neuropsychopharmacology, 21(2S), 16-23. doi: 10.1016/S0893133X(98)00135-3

American Psychiatric Association. (2013). Diagnostic and statistical manual of mental disorders (5th ed.). [Kindle DX version]. Retrieved from Amazon.com.

Andersen, S. M., Baum, A. (1994). Transference in interpersonal relations: Inference and affect based on significant-other representations. Journal of Personality, 62(4), 459-497. doi: 10.1111/j.1467-6494.1994.tb00306.x

Anderson, W. L. (2002) Free spirits, presumptions, women, and false prophets: The discernment of spirits in the later Middle Ages. PhD. Dissertation. University of Chicago.

Asclepius. (n.d.). Retrieved from https://en.wikipedia.org/wiki/Asclepius.

Barnett, M. (2007). What brings you here? An exploration of the unconscious motivations of those who choose to train and work as psychotherapists and counsellors. Psychodynamic Practice, 13(3), 257-274. doi: 10.1080/14753630701455796

Barnes, L.L., Plotnikoff, G.A., Fox, K., and Pendleton, S. (2000). Spirituality, religion, and pediatrics: Intersecting worlds of healing. Pediatrics. 106 (4), 899-908.

Baumeister, R. F., Masicampo, E. J., Vohs, K. D. (2010). Do conscious thoughts cause behavior? Annual Review of Psychology, 62, 331-361.

Beauregard, M. (2014). The primordial psyche. Journal of Consciousness Studies, 21(7-8), 132157.

Beauregard, M. (2012). Brain wars: The scientific battle over the existence of the mind and proof that will change the way we live our lives. [Kindle DX version]. Retrieved from Amazon.com. 
Beauregard, M. (2011a). Transcendent experiences and brain mechanisms. In E. Cardeña, \& M. Winkelman Editors (Eds.), Altering consciousness. Multidisciplinary perspectives.

Volume 2. Biological and psychological perspectives. (63-84). ABC-CLIO.

Beauregard, M (2011b). Neuroscience and spirituality: findings and consequences. In H. Walach et al. (Eds.), Studies in Neuroscience, Consciousness and Spirituality. (57-73). Springer Science \& Business Media.

Beauregard, M., \& Paquette, V. (2006). Neural correlates of a mystical experience in Carmelite nuns. Neurosci Lett, 405(3), 186-190. doi: 10.1016/j.neulet.2006.06.060

Beischel, J., Rock, A. J., \& Krippner, S. (2011). Altered consciousness from the age of enlightenment through mid-20 ${ }^{\text {th }}$ century. In E. Cardeña, \& M. Winkelman Editors (Eds.), Altering consciousness. Multidisciplinary perspectives. Volume 1. History, Cutlure and the Humanities. (63-84). ABC-CLIO.

Berzoff, J., Flanagan, L. M., \& Hertz, P. (2011). Inside out and outside in: Psychodynamic clinical theory and psychopathology in contemporary multicultural contexts. (3rd ed.) [Kindle DX version]. Plymoth, UK: Rowman \& Littlefield Publishers, Inc.

Bevan, M. E. (2014). A method of phenomenological interviewing. Qualitative Health Research 24(1), 136-144.

Bourguignon, E. (1989). Trance and shamanism: What's in a name? Journal of Psychoactive Drugs, 21(1). doi: 10.1080/02791072.1989.10472138

Bourguignon, E. 1974. Culture and the Varieties of Consciousness. Reading, Massachusetts: Addison-Wesley.

Bourguignon, E. (1968). A cross-cultural study of dissociational states. Research Foundation, Ohio State University.

Bowie, R. (2000). The anthropology of religion. Oxford: Blackwell. 
Block, N. (2001). Paradox and cross purposes of recent work on consciousness. Cognition, 79, 197-219. doi: 10.1016/S0010-0277(00)00129-3

Bohm, D. (1980). Wholeness and the implicit order. New York: NY: Routledge.

Brinton, D. G. (1894). Nagualism: A study in Native Amercian folk-lore and history. Philadelphia: MacCalla \&Company.

Bruhl, L. (1926/1966). How natives think. Washington Square Press: New York.

Cardeña, E. (2011a). Altering consciousness: Setting up the stage. In E. Cardeña, \& M. Winkleman (Eds.), Altering consciousness multidisciplinary perspectives volume 1: History culture and the humanities. (pp. 1-22). Santa Barbara: California: ABC-CLIO, LLC.

Cardeña, E. (2011b). Altered consciousness in emotion and psychopathology. In E. Cardeña, \& M. Winkelman Editors (Eds.), Altering consciousness. Multidisciplinary perspectives. Volume 2. Biological and psychological perspectives. (279-300). ABC-CLIO.

Cardeña, E. (2005). The phenomenology of deep hypnosis: Quiescent and physically active. Journal of Clinical and Experimental Hypnosis, 53, 37-59. doi:

$10.1080 / 00207140490914234$

Cahn, B. R., \& Polich, J. (2006). Meditation states and traits: EEG, ERP, and neuroimaging studies. Psychol Bull, 132(2), 180-211. doi: 10.1037/0033-2909.132.2.180

Campbell, J.L., Qunicy, J., Osserman, J., and Pederson, O. K. (2003). Coding in-depth semi-structured interviews: Problems of unitization and intercoder reliability and agreement. Sociological Methods and Research, 42 (3), 294-320.

doi: $10.1177 / 0049124113500475$

Chalmers, D. J. (1997). The Conscious Mind: In Search of a Fundamental Theory: OUP USA. 
Chalverat, C. (2003). La dynamique de l'archétype "guérisseur- blessé” à l'œuvre dans la pratique et la formation des praticiens de l'aide. Education Permanete (3), 22-25.

Cohen, C.B., Wheeler, S.E., Scott, D.A., \& The Anglican Working Group in Bioethics. (2001). Walking a fine line: Physician Inquiries into patients' religious and spiritual beliefs. The Hastings Center Report. 31 (5), 29-39.

Connelly, R., \& Light, K. (2003). Exploring the "New" Frontier of Spirituality in Health Care: Identifying the Dangers. J Relig Health, 42(1), 35-46.

Conti-O'Hare, M. (2002). The nurse as wounded healer: From trauma to transcendence. Jones \& Bartlett Learning.

Courtois, C. A., Ford, J. D., Herman, J. L., \& van der Kolk, B. A. (2009). Treating Complex Traumatic Stress Disorders (Adults): Scientific Foundations and Therapeutic Models [Kindle DX version]: Guilford Publications.

Clottes, J. \& Lewis-Williams, D. (1998). The Shamans of Prehistory: Trance and Magic in the Painted Caves. New York: Harry N. Adams.

Coady, N., \& Hill, G. (2003). Comparing euro-western counselling and aboriginal healing methods. Native Social Work Journal, 5, 44-63.

Cook, A., Spinozzola, J., Ford, J., Lanktree, C., Blaustein, M., Cloitre, M., DeRosa, R., Hubbard, R., Kagan, R., Liautaud, J., Mallah, K., Olafson, \& van der Kolk B. (2005). Complex trauma in children and adolescents. Psychiatric Annals, 35(5), 390-398. doi: $10.3928 / 00485713-20050501-05$

Craffert, P., F. (2011). Shamanism and the shamanic complex. Biblical theology bulletin. 41(3). 151-161. doi: 10.1177/0146107911413212

Creswell, W. (2013). Qualitative inquire \& research design: Choosing among five approaches. [Kindle DX version]. Retrieved from Amazon.com. 
Cropper, M. (2002). The life of Evelyn Underhill: An intimate portrait of the groundbreaking author of mysticism. Woodstock, Vermont: Skylight Paths Publishing.

Devereux, G. (1961). Shamans as neurotics. American Anthropologist, 63(5), 1088-1090. doi: 10.1525/aa.1961.63.5.02a00110

Devinsky, O., \& Lai, G. (2008). Spirituality and religion in epilepsy. Epilepsy Behav, 12(4), 636 643. doi: 10.1016/j.yebeh.2007.11.011

de Rios, M. D. D., \& Winkelman, M. (1989). Shamanism and altered states of consciousness: An Introduction. Journal of Psychoactive Drugs, 21(1), 1-7. doi: $10.1080 / 02791072.1989 .10472137$

Di Agresti, D. (1980). Svilupi della riforma monastic Savonaroliana [Developments in the Savonrolan monastic reform]. Florence: Olschki.

Dietrich, A. (2003). Functional neuroanatomy of altered states of consciousness: The transient hypofrontality hypothesis. Conscious Cogn, 12(2), 231-256. doi: 10.1016/s1053

Dossey, B. M., Keegan, L., \& Guzetta, C. E. (2005). Holistic Nursing: A Handbookfor Practice. ( $4^{\text {th }}$ ed.) Sudbury : Mass, Jones \& Bartlett Publishers.

Dossey, L. (1995). Whatever happened to healers? Alternative Therapies in Health and Medicine, 1(5), p. 6-13.

Dowling, M. (2007). From Husserl to van manen. A review of different phenomenological approaches. International Journal of Nursing Studies (44). doi: 131-142.8100(02)00046 6

Dunning, T. (2006). Caring for the wounded healer-nurturing the self. Journal of Bodywork and Movement Therapies, 10(4), 251-260. doi:10.1016/j.jbmt.2005.05.001

Egnew, T. R. (2005). The meaning of healing: transcending suffering. Ann Fam Med, 3(3), 255262. doi: 10.1370/afm.313 
Eger, S. (1978). Huichol women's art. In K. Berin (Ed.), Art of Huichol Indians (pp. 35-53).

New York: Fine Arts Museums of San Francisco/Harry n. Abrams.

Eliade, M. (1964/2004). Shamanism: archaic techniques of ecstasy. Princeton, NJ: Princeton University Press.

Etic. (n.d.). In. Webster Dictionary online. Retrieved from https://www.merriamwebster.com/dictionary/etic

Farber, B. A., Manevich, I., Metzger, J., Saypol, E. (2005). Choosing psychotherapy as a career: Why did we cross that road? Journal of Clinical Psychology 61, 1009-1031. doi: 10.1002/jclp.20174

Flaherty, G. (1992). Shamanism in the eighteenth century. Princeton, NJ: Princeton University Press.

Frank, J. D., \& Frank, J. B. (1993). Persuasion and Healing: A Comparative Study of Psychotherapy ( $3^{\text {rd }}$ ed.). Baltimore, Maryland: Johns Hopkins University Press.

Freshwater, D. (1995). Polarity and unity in caring the healing power of symptoms. Complementary Therapies in Nursing and Midwifery, 5(5), 136-139. doi:10.1016/S13536117(99)80092-4

Fischer, R. (1981). Matters mastermind: The model making brain as analogy. Diogenes, 29 (116), 18-39.

Fischer, R. (1986). Toward a neuroscience of self-experience and states of self-awareness and interpreting interpretations. In B.B. Wolman \& M. Ullman (Ed.). Handbook of states of consciousness. (pp. 3-30). U.S.A: Nostrand Reinhold Company Inc.

Fischer, R. (1992). A cartography of cognitive and non-cognitive states of consciousness. Anthropology of Consciousenss (3) 3-4. 3-13. 
Friedrich, W. N. (2002). Psychological Assessment of Sexually Abused Children and Their Families: SAGE Publications.

Gackenbach, J., \& LaBerge, S. (1988). Conscious mind, sleeping brain: perspectives on lucid dreaming: Plenum Press.

Gilbert, C. (1995). Optimal physical performance in athletes: Key roles of dopamine in a specific neurotransmitter/hormonal mechanism. Mechanics of Ageing and Development, 1, 83-102. doi: dx.doi.org/10.1016/0047-6374(95)01635-X

Girand, T. A., Martius, D. L., \& Cheyne, J. A. (2007). Mental representation of space: Insights from an oblique distribution of hallucinations. Neurospychologia, 45, 1257-1269.

Glaser, G. H. (1978). Epilepsy, hysteria, and “possession”. The Journal of nervous and mental disease, 166(4), 268-274.

Greeley, A. M. (1975). The sociology of the paranormal: A reconnaissance (Sage Research Papers in the Social Sciences, Vol. 3, Series No. 90-023). Beverly Hills, CA: Sage.

Grof, S. (1985). Beyond the brain: Birth, death and transcendence in psychotherapy. Albany NY: State University of New York Press.

Grof, S., \& Grof, C. (1989). (Eds.). Spiritual emergency: When personal transformation becomes a crisis. Los Angeles: Tarcher.

Groseback, J. (1975). The archetypal image of the wounded healer. Journal of analytic psychology. 20(2). doi:org/10.1111/j.1465-5922.1975.00122.x

Guggenbühl-Craig, A. (1971). Power in the helping professions. Thompson, Conn. Spring Publications.

Hamayon, R. N. (2001). Shamanism: Symbolic system, human capability and western ideology. In H. Francfort, \& R. Hamayon (Eds.), The concept of shamanism: Uses and abuses. (pp. 1-27). Budapest: Akadémiai Kiadó. 
Harner, M. J. (1962). Jivaro souls. American Anthropologist, 64(2), 258-272. doi: 10.1525/aa.1962.64.2.02a00020

Harner, M. (1980/1990). The way of the shaman. (2nd ed.) New York: Harper Collins.

Harner, S., \& Tyron, W. (1992). Psychoimmunological effects of shamanic drumming. In M. Hoppal \& J. Pentikainen (Eds.), Northern Religions and Shamanism. (pp.196-204) Budapest, Hungary: Akademial Klado, Helsinki Finnish Literature Society.

Harner Shamanic Counselling. (www.shamanism.org).

Harris, C. D. (2005). Neurophysiology of sleep and wakefulness. Respiratory Care Clinics of North America, 11, 567-586.

Hayes, J. A. (2002). Playing with fire: Countertransference and clinical epistemology. Journal of Contemporary Psychotherapy, 32(1), 93-100.

Haynes, J. D., Deichmann, R., and Rees, G. (2005) Eye-specific suppression in human LGN reflects perceptual dominance during binocular rivalry. Nature 438, 496-9.

Heidegger, M. (1927/1962). Being and time. New York: Harper.

Hill, P. C., Pargament, K. I., Hood Jr., R. W., Mccullough, M. E., Swyers, J. P., Larson, D. B., Zinnbayer, B. J. (2000). Conceptualizing religion, and spirituality: Points of commonality, points of departure. Journal for the Theory of Social Behaviour, 30(1), 5177.

Hufford, D. J. (1985). Commentary: Mystical experience in the modem world. In G. W. Foster, The world was flooded with light: A mystical experience remembered (pp. 87-183). Pittsburgh, PA: University of Pittsburgh Press.

Hulkrantz, A. (1985). The shaman and the medicine-man. Social Science and medicine, 20(5), 511-515. doi: 10.1016/0277-9536(85)90367-3 
Hunt, H. T. (2012). A collective unconscious reconsidered: Jung's archetypal imagination in the light of contemporary psychology and social science. Journal of analytical psychology, 57, 76-98. doi: 10.1111/j.1468-5922.2011.01952.x

Husserl, E. (1960/1982). Cartesian meditations. An introduction to phenomenology. (Cairns, D. Trans.). The Hague: Maritnus Niijhoff Publishers.

'lolana, G. (2016). Jung and goddess: The significance of jungian and post-jungian theory to the development of the western goddess movement. (Unpublished doctoral dissertation). School of Critical Studies, College of the Arts, University of Glasgow.

Jackson, S. W. (1992). The Listening Healer. American Journal of Psychiatry, 149(12), 16231632.

Jacobson, K. H. (1993). Organization and the mother archetype: A jungian analysis of adult development and self-identity within the organization. Administration \& Society. 25(1), $60-84$.

James, W. (1842/1986). Essays in Psychical Research. (Vol. 16). USA: Harvard University Press.

James, W. (1905/2008). The Varieties of Religious Experience: A Study in Human Nature. (15th ed.). USA: Arc Manor.

James, W. (1910). Psychology. New York: Henry Holt and Company.

James (n.d.) In Standford Encyclopaedia Online. Retrieved from http://plato.stanford.edu/entries/james/\#5.

Jeste, D. V., del Carmen, R., Lohr, J. B., \& Wyatt, R. J. (1985). Did Schizophrenia exist before the eighteenth century? Comprehensive Psychiatry, 26(6), 493-503. doi: 10.1016/0010$440 X(85) 90016-1$

Jones, P. N. (2006). Shamanism: An inquiry into the history of the scholarly use of the term in 
english-speaking north america. Anthropology of Consciousness, 17(2), 4-32. doi: 10.1525/ac.2006.17.2.4

Joulin, S. (2015). Vers un traitement plus holistique des personnes ayant abuse sexuellement de mineurs: Analyse herméneutique et qualitative de la dimension religeuse et spirituelle de distorsions cognitives liées à l'abus et de leur prise en compte dans la psychothérapie (Unpublished doctoral dissertation). Saint Paul University, Ottawa, ON, Canada.

Jung, C. G. (1916). Psychology of the unconscious: A study of the transformations and symbolism of the libido, a contribution to the history of the evolution of thought. New York: Moffat Yard and Company.

Jung, C. G. (1933/2001). Modern man in search of a soul. New York: Routledge.

Jung, C. G. (1953). The collected works of C. G. Jung, Volume 7. (2 ${ }^{\text {nd }}$ ed.) Princeton: Princeton University Press.

Jung, C. G. (1954). The collected works of C. G. Jung, Volume 8. (2 $2^{\text {nd }}$ ed.) Princeton: Princeton University Press.

Jung, C. G. (1959a/1980). Collected works of the unconscious, Volume 9 (Part 1). (2nd ed.). [Kindle DX version]. Retrieved from Amazon.com.

Jung, C. G. (1959b/1980). Collected works of the unconscious, Volume 9 (Part 2). (2nd ed.). [Kindle DX version]. Retrieved from Amazon.com.

Jung, C. G. (1960). Synchronicity. New York: NY: Princeton University Press.

Jung, C. G. (1961/1990). Memories, dreams, reflections. New York: Random House.

Jung, C. G., von Franz, M. L., Henderson, J. L., Jacobi, J., Jaffé, A. (1964/2010). (3rd ed.) Man and His Symbols: Random House Publishing Group.

Jung, C. G. (1967/1970). The collected works of C.G. Jung, Volume 13: Alchemical studies. 
Princeton: Princeton University Press.

Jung, C. G. (1968). The collected works of C. G. Jung, Volume 12: Psychology and alchemy: Princeton: Princeton University Press.

Jung, C. G. (1969) The collected works of C.G. Jung, Volume 11: Psychology of religion: West and East. Princeton: Princeton University Press.

Jung, C. G. (1971/1991). Psychological types. Princeton University Press: London.

Jung, C. G. (1971). The Portable jung. New York: Penguin Books.

Kalsched, D. (1996). The inner world of trauma: Archetypal defenses of the personal spirit. Routledge: New York.

Keagan, L. (1994). The nurse as healer. Delmar Publishers: University of Michigan.

Kehoe, A.B., 1996. Eliade and Hultkrantz: the European primitivism tradition. American Indian Quarterly 20, 377-392. doi: 10.2307/1185783

Kehoe, A.B., 2000. Shamans and Religion: An Anthropological Exploration in Critical Thinking. Waveland Press, Inc., London.

Kenny, G. (2012). The healers journey: a literature review. Complement Ther Clin Pract, 18(1), 31-36. doi:10.1016/j.ctcp.2011.08.002

Kirmayer, L. (1993). Healing and the invention of metaphor: The effectiveness of symbols revisited. Cultural Medical Psychiatry, 17, 191-195.

Kirmayer, L. (2003). Asklepion dreams: The ethos of the wounded-healer in the clinical encounter. Transcultural Psychiatry. 40(2), 248-277. doi: 10.1177/1363461503402007

Kleinman, A. M. (1973). Some issues for a comparative study of medical healing. International Journal of Social Psychiatry, 19(3-4), 159-165.

Knox, J. (2003). Archetype, attachment, analysis: Jungian psychology and the emergent mind. [Kindle DX version]. Retrieved from Amazon.com. 
Koenig, H.G. (2000). Religion, spirituality, and medicine: Application to clinical practice.

JAMA. 284 (13), 1708. doi: 10.1001/jama.284.13.1708-JMS1004-5-1

Krippner, S. (1999). The varieties of dissociative experience: A transpersonal, post-modern model. International Journal of Transpersonal Studies. 18(2), 81-101.

Krippner, S. (2000). The Epistemology and Technologies of Shamanic States of Consciousness. Journal of Consciousness Studies, 12(11), 93-118.

Krippner, S. (2002). Conflicting Perspectives on Shamans and Shamanism Points and Counterpoints. American Psychologist, 57(11), 962-977. doi: 10.1037/0003 066X.57.11.962

Krippner, S. (2012). Shamans as healers, counselors, and psychotherapists. International Journal of Transpersonal Studies, 31(2), 72-79.

Kroll, J., \& Bachrach, B. (1982). Visions and psychopathology in the middle ages. The Journal of Nervous and Mental Disease, 170(1), 41-50. doi: 10.1097/00005053-19820100000007

Laughlin, C. McManus, J., \& d'Aquili, E. (1990). Brain, symbol, and experience. Toward a Neurophenomenology of consciousness. New York: Columbia University.

Lanza, R., \& Berman, R. (2009). Biocentrism: How life and consciousness are the keys to understanding the true nature of the universe. Dallas, TX: BenBella Books, Inc.

Levine, J., \& Steele, L. (2005). The transcendent experience: conceptual, theoretical, and epidemiologic perspectives. Explore (NY), 1(2), 89-101. doi:

10.1016/j.explore.2004.12.002

Livio, M. (2005). The equation that couldn't be solved. How mathematical genius discovered the language of symmetry. New York, NY. Simon and Schuster.

'lolana, P. (2016). Jung and goddess: The significance of Jungian and post-jungian theory to the 
development of the western goddess movement. (Unpublished doctoral dissertation). School of Critical Studies, College of the Arts, University of Glasgow.

Lombard, M., Snyder-Dutch, J., Bracken, C. C. (2004). Practical resources for assessing and reporting intercoder reliability in content analysis research projects. Intercoder Reliability in Content Analysis, 1-18.

Lombard, M., Snyder-Dutch, J., Bracken, C. C. (2002). Content analysis in mass communication assessment and reporting of intercoder reliability. Human Communication Research, 28(4), 587-604.

Ludwig, A. M. (1966). Altered states of consciousness. Archives of General Psychiatry, 15, 225-234. Available from http://dx.doi.org/10.1001/archpsyc.1966.01730150001001

Maclean, P. (1973). The triune concept of brain and behavior. Toronto: University of Toronto Press.

Magico-religious practitioner (n.d.). In Merriam-Webster online. Retrieved from https://www.merriam-webster.com/dictionary/magico-religious

Mandell, A. J. (1980). Toward a psychobiology of transcendence: God in the brain (pp. 379464). Springer US.

Mammalian brain. (n.d.) In: The Free Dictionary online. Retrieved from http://www.thefreedictionary.com/mammalian+brain

Manchester, C. F., Allen, T., \& Tachiki, K. H. (1998). Treatment of dissociative identity disorder with neurotherapy and group self-exploration. Journal of Neurotherapy, 2(4), 40-53. doi: 10.1300/J184v02n04_03

Mazzola, L. C. (1998). The Medicine Wheel: Centre and Periphery. Journal of Popular Culture. doi: 10.1111/j.0022-3840.1988.2202_63.x 
Means, J. J. (2002). Mighty Prophet/Wounded Healer. Journal of Pastoral Care, 56(1), 41-49.

Merchant, J. (2012). Shamans and analysts: New insights on the wounded healer. [Kindle DX version]. Retrieved from Amazon.com.

Menezes, Jr. A., \& Moreira-Almedia, A. (2009). Differential diagnosis between spiritual Experiences and mental disorders of religious content. Psiq Clin, 36, 75-82.

Menezes Jr, A., \& Moreira-Almeida, A. (2010). Religion, spirituality, and psychosis. Current psychiatry reports, 12(3), 174-179. doi: 10.1007/s11920-010-0117-7

McGilchrist, I. (2009). The master and his emissary: The divided brain and the making of the western world. [Kindle DX version]. Retrieved from Amazon.com.

Mishara, A. L., \& Schwartz, M. (2011). Altered states of consciousness as paradoxically healing: An embodied social neuroscience perspective. In E. Cardeña, \& M. Winkelman Editors (Eds.), Altering consciousness multidisciplinary perspectives. Volume 2. (327-254).

\section{ABC-CLIO.}

Miller, G. D., \& Baldwin Jr, D. C. (1987). Implications of the wounded-healer paradigm for the use of the self in therapy. Journal of Psychotherapy \& the Family, 3(1), 139-151.

Moustakas, C. (1994). Phenomenological research methods. Thousand Oaks, CA: Sage.

Mousalimas, S. (1990). The concept of participation in Lévy-Bruhl's 'primitive mentality. Jaso 21(1), p. 33-46.

Mouter, N., \& Vonk Noordegraaf, D. (2012). Intercoder reliability for qualitative research: You win some, but do you lose some? The Netherlands: Delft University of Technology, 1-9.

Moutoussis, K. and Zeik, S. (2002) The relationship between cortical activation and perception investigated with invisible stimuli. Proceedings of the National Academy of Sciences 99: $14,9527-32$.

Neumann, E. (1954/2002). The origins and history of consciousness. Routledge: New York. 
New Age. (n.d.). Retrieved from https://www.britannica.com/topic/New-Age-movement.

Newberg, B., Alai, A., Baime, M., Pordehnad, M., Santanna, J. and d'Aquilli, E. (2001). The measurement of a regional cerebral blood flow during the complex cognitive task of meditation: A preliminary SPECT study. Psychiatry Research: Neuroimaging Section 106, 113-122.

Newberg, B., Lee, Y. L. (2005). The neuroscientific study of religious and spiritual phenomena: Or why god doesn't use biostatistics. Zygon, 2(40), p. 469-490.

Noirhomme, Q., Laureys, S. (2011). Altering Consciousness and Neuropathology. In E. Cardeña \& M. Winkleman (Eds.), Alterning consciousness multidisciplinary perspectives volume 2: History culture and the humanities. (pp. 263-278). Santa Barbara: California: ABCCLIO, LLC.

Noll, R. (1983). Shamanism and schizophrenia: a state-specific approach to the "schizophrenia metaphor" of shamanic states. American Ethnologist, 10(3), 443-459. doi: 10.1525/ae.1983.10.3.02a00030

Nouwen, H. (1972). The wounded healer: Ministry in contemporary society. New York: Crown Publishing Group.

Ogembo, J. M. (2005). The Persisting Conflict of Interpretations of Shamanism. Reviews in Anthropology, 34(2), 197-210. doi: 10.1080/00938150590948649

Overton, J. A. (1998). Shamanism and clinical hypnosis. A brief comparative analysis. Shaman, 6(1), 1-19. Retrieved from http://www.folkscene.hu/magzines/shaman/c6.html Pargament, K. I. (2007). Spiritually integrated psychotherapy: Addressing the sacred. New York: The Guilford Press.

Parrott, A. C. (2001). Human psychopharmacology of Ecstasy (MDMA): a review of 15 years of empirical research. Hum Psychopharmacol, 16(8), 557-577. doi: 10.1002/hup.351 
Paleomammalian (n.d.). In wiktionary online. Retrieved from http://en.wiktionary.org/wiki/paleomammalian

Paloutzian, R. F. \& Park, C. L. (2005). Handbook of the psychology of religion and spirituality. New York: The Guilford Press.

Parry, G. A. (2006). Native wisdom in a quantum world. Shift at the Frontiers of Consciousness, 9, 29-33.

Peters, L. G., \& Price-Williams, D. (1980). Towards an experiential analysis of shamanism. The American Anthropological Association, 7(3), 397-418. doi: doi.org/10.1525/ae.1980.7.3.02a00010

Peters, L. G. (1982). Trance, initiation, and psychotherapy in Tamang shamanism. Amercian Ethnologist, 9(1,), 21-46.

Presti, D. E. (2011). Neurochemistry and Altered Consciousness. In E. Cardeña, \& M. Winkelman Editors (Eds.), Altering consciousness multidisciplinary perspectives. Volume 2: Biological Perspectives. (pp. 21-42). Santa Barbara: California: ABC-CLIO, LLC.

Previc, F. H. (2011). Dopamine, altered consciousness, and distant space with special reference to shamanic ecstasy. In E. Cardeña \& M. Winkleman (Eds.), Altering consciousness multidisciplinary perspectives volume 2: Biological Perspectives. (pp. 43-62). Santa Barbara: California: ABC-CLIO, LLC.

Previc, F. H. (2009). The dopaminergic mind in human evolution and history. Cambridge, U.K.: Cambridge University Press.

Postmodernism. (n.d.) Retrieved from https:/www.britannica.com/topic/postmodernismphilosophy.

Putman, F. W. (2005, November). States of Being. Invited address presented at the 22nd Annual Meeting of the International Society for the Study of Dissociation, Toronto, Canada. 
Revonsuo, A., Kallio, S., \& Sikka, P. (2009). What is an altered state of consciousness? Philosophical Psychology, 22(2), 187-204. doi: 10.1080/09515080902802850

Riffe, D., Lacy, S., Fico, F. G. (2005). Analyzing media messages: Using quantitative content in analysis in research. Mahway, NJ: Lawrence Erlbaum Associaties.

Richtand, N. M., \& McNamara, R. K. (2008). Serotonin and dopamine interactions in psychosis prevention. Progress in brain research, 172, 141-153.

doi:10.1016/S00796123(08)00907-2

Rock, A. J.., \& Krippner, S. (2011). Demystifying shamanism and their world: A multidisciplinary study. [Kindle DX version]. Retrieved from Amazon.com.

Rosen, D. H., Smith, S. M., Huston, H. L., Gonzalez, G. (1991). Empirical study of associations between Symbols and their Meanings: Evidence of Collective Unconscious (archetypal) memory. Journal of Analytical Psychology, 36, p. 211-228.

Rouget, G. (1985). Music and Trance: A Theory of the Relations Between Music and Possession. Chicago: University of Chicago Press.

Roy, L. (1942/2001). Transcendent Experiences: Phenomenology and Critique: University of Toronto Press.

Ryan, R. E. (1999) The strong eye of the shaman. Inner Traditions: Rochester, Vermont.

Salamone, J., and Correa, M. (2002). Motivational views of reinforcement: implications for understanding behavioral functions of nucleus accumbens dopamine. Behavioral Brain Research, 137, 3-25. doi: 10.1016/S0166-4328(02)00282-6

Sansonese, J. N. (1994). The body of myth: Mythology, shamanic trance, and the sacred geography of the body. Rochester, VM. Inner Traditions/Bear \& Co.

Sandner, D. F; \&Wong, S. H. (1997) The sacred Heritage: The influence of shamanism on analytic psychology. New York: NY: Routledge. 
Sartre, J. P. (1943/2005). Being and nothingness. (Barnes, H. Trans.). London: Routledge.

Sartre, J. P. (1960). The transcendence of the ego: An existential theory of consciousness.

(Williams, F., Kirpatrick, R. Trans.). New York: Hill and Wang.

Sedgwick, D. (1994). The wounded healer: Countertransference from a Jungian perspective. New York: Routledge.

Seidman, I. (2013). Interviewing as qualitative research: A guide for researchers in education and the social sciences ( $4^{\text {th }}$ ed.). New York: Teachers College Press. Retrieved from Amazon.ca.

Schaefer, S. B. (2011). Peyote and meaning. In E. Cardeña, \& M. Winkleman (Eds.), Altering consciousness multidisciplinary perspectives volume 2: Biological Perspectives. (pp. 147-166). Santa Barbara: California: ABC-CLIO, LLC.

Scott, E. (2014). The Visionary Psyche: Jung's Analytical Psychology and Its Impact on Theories of Shamanic Imagery. Anthropology of Consciousness. 25 (1), 91-115. doi: 10.1111/anoc. 12020

Shephard, P. A. (1999). Seeing with the Strong Eye: Psychotherapist Initial Experience of Shamanic Journeying. (PhD Psychology Doctoral), Massachusetts School of Professional Psychology.

Sierra, M., \& Berrios, G. E. (1998). Depersonalization: neurobiological perspectives. Biological psychiatry, 44(9), 898-908. doi: 10.1016/S0006-3223(98)00015-8

Sidky, H. (2010). On the antiquity of shamanism and its role in human religiosity. Method and theory in the study of religion, 22, 68-92. doi: 10.1163/094330510X12604383550963

Silverman, J. (1967). Shamans and acute schizophrenia. American anthropologist, 69(1), 21 31. doi: 10.1525/aa.1967.69.1.02a00030 
Sluhovsky, M. (2011). Spirit Possession and Other Alterations of Consciousness. In E. Cardeña \& M. Winkleman (Eds.), Altering consciousness multidisciplinary perspectives volume1: History, Culture and the Humanities. (pp. 73-88). Santa Barbara: California: ABC-CLIO, LLC.

Smith, M. C. (2007). Jung and shamanism in dialogue. Indiana: USA: Trafford Publishing.

Smith, A. P. (2009). A history of gnosticism. Wheaton, IL: Quest Books.

Sotirova-Kohli, M., Opwis, K., Roesler, C., Smith, S. M., Rosen, D. H., Vaid, J., \& Djonov, V. (2013). Symbol/Meaning Paired-Associate Recall: An “Archetypal Memory” Advantage?. Behavioral sciences, 3(4), 541-561. doi:10.3390/bs3040541

Stace, W. T. (1972). Teachings of the Mystics: Penguin Group (USA) Incorporated.

Stevens, A. (1994). Jung: A very short introduction. [Kindle DX version]. Retrieved from Amazon.com.

Storr, A. (1989). Freud: A very short introduction. [Kindle DX version]. Retrieved from Amazon.com.

Tart, C. T. (1975). States of consciousness: EP Dutton: New York.

Tart, C. T. (1986). Consciousness, Altered States, and Worlds of Experience. Journal of Transpersonal Psychology, 18(2), 159-172.

Taylor, E. (1983). William James on exceptional mental states. The 1896 Lowell lectures. New York: Scribner.

Underhill, E. (1930). Mysticism: A study in the nature and development of man's spiritual consciousness. [Kindle DX version]. Retrieved from Amazon.com.

University of Minnesota. The Foundations of Shamanism and Shamanic Healing (https://www.csh.umn.edu/node/1476). 
VanPool, C. S. (2009). The signs of the sacred: Identifying shamans using archaeological evidence. Journal of Anthropological Archaeology, 28(2), 177-190. doi: 10.1016/j.jaa.2009.02.003

van Manen, M. (1990). Researching lived experience: Human science for an action sensitive pedagogy. Albany: State University of New York Press.

Vaitl, D., Birbaumer, N., Gruzelier, J., Jamieson, G. A., Kotchoubey, B., Kubler, A., ... Weiss, T. (2005). Psychobiology of altered states of consciousness. Psychol Bull, 131(1), 98127. doi: $10.1037 / 0033-2909.131 .1 .98$

Varela, F., Lachaux, J. P., Rodriguez, E., \& Martinerie, J. (2001). The brainweb: phase synchronization and large-scale integration. Nature reviews neuroscience, 2(4), 229-239.

Veltmeyer, M. D., McFarlane, A. C., Bryant, R. A., Mayo, T., Gordon, E., \& Clark, C. R. (2006). Integrative assessment of brain function in PTSD: brain stability and working memory. Journal of integrative neuroscience, 5(1), 123-138. doi: 10.1142/S0219635206001057

Villoldo, A. (2000). Shaman, healer, sage. New York: Harmony Books.

Vitebsky, P. (1995). The shaman: Voyages of the soul, trance, ecstasy and healing from Siberia to the Amazon, London: Duncan Baird.

Walker, M. (2008). Symmetry, science, and shamanism: Towards a theory of everything. Horizons, 27-55.

Walsh, R (1989). What is a shaman? Definition, origin and distribution. Journal of Transpersonal Psychology, 21,1-11.

Walsh, R. (1994). The making of a shaman: calling, training, and culmination. Journal of Humanistic Psychology. doi: 10.1177/00221678940343003

Walsh, R. (1995). Phenomenological mapping: A method for describing and comparing states of consciousness. Journal of Transpersonal Psychology, 27, 25-38. 
Walsh, R. (2007). The world of shamanism: New views of an ancient tradition. [Kindle DX version]. Retrieved from Amazon.com.

Whitehead, C. (2011). Altered consciousness in society. In E. Cardeña, \& M. Winkleman (Eds.), Altering consciousness multidisciplinary perspectives volume 1: History, Culture, and the Humanities. (pp. 181-202). Santa Barbara: California: ABC-CLIO, LLC.

Wilbur, K. (1997/1993). The spectrum of consciousness. Wheaton, IL: Quest Books. Retrieved from Amazon.ca.

Winkelman, M. (1984). A Cross Cultural Study on Magico-Religious Practitioners. (PhD), University of California, Irvine, California.

Winkelman, M. (1986). Magico-religious practitioner types and socioeconomic conditions. Behavior Science Research, 20, 17-46.

Winkelman, M. (2010). Shamanism: A biopsychosocial paradigm of consciousness and healing. [Kindle DX version]. Retrieved from Amazon.com.

Wolf, F. A. (1991). The eagle's quest: A physicist finds scientific truth at the heart of the shamanic world. New York, NY: Simon and Shuster.

Wolf, F. A. (1994). The dreaming universe: A mind-expanding journey in the realm where psyche and physics meet. New York: NY: Simon and Schuster.

Wolf, F. A. (2001). Mind into matter: A new alchemy into mind and spirit. Portsmouth, NH: Moment Point Press, Inc.

Wolf, F. A. (2004). The yoga of time travel: How the mind can defeat time. Wheaton, Illinois: Theosophical Publishing House.

Wulf, D. M. (2000). Mystical experience. In E. Cardeña, S. J. Lynn, \& S.S. Krippner (Eds.). Varieties of anomalous experience (pp. 397-440). Washington DC: American Psychological Association. 
Young-Eisendrath, P., \& Dawson, T. (1997). The cambridge companion to jung. Cambridge: Cambridge University Press.

Yensen, R. \& Dryer, D. (1996). The Consciousness research of Stanislav Grof: A cosmic portal beyond individuality. In B. W. Scotton, Chinen, A. B. \& Battsta, J. R. (Eds.). Textbook of transpersonal psychiatry and psychology. New York, NY: Basic Books.

Zerubavel, N., \& Wright, M. O. (2012). The dilemma of the wounded healer. Psychotherapy (Chic), 49(4), 482-491. doi:10.1037/a0027824 


\section{Notes}

${ }^{1}$ The paleomammalian brain is part of the limbic system and contains the processes involved in social behavior and nonverbal, emotional, and analogical information processing, such as mediating affect, sex, fighting/self-defence, social relations, bonding and attachment. The neomammilian brain (frontal lobes or neocortical structures) is involved in language, rational thought, analytic process and complex problem solving (Winkelman, 2010). The reptilian brain (R-complex) is part of the upper spinal cord, and lower brain structures that extend into the thalamus and hypothalamus beneath the temporal lobe. It is responsible for metabolism, digestion, and respiration, wakefulness, attention, and regulation and coordination of behaviour (Winkelman, 2010).

${ }^{2}$ For example, for consciousness to occur, a synchronization of brain states is thought to incorporate information into a common workspace, involving cortico-cortical and thalamocorticoal functional connections with other cerebral areas of the brain that allow for synchronization of neural networks to occur (Noirnomme \& Laureys, 2011)

${ }^{3}$ Intercoder reliability should be designed to include multiple coders for both the content and the assessment along with the reporting of intercoder reliability among them. Reliability is necessary for validity in the study and without it the results and conclusions in the research project may be suspect (Mouter \& Vonk Norrdiegraaf, 2012). The steps to conduct intercoder reliability include: (1) Determining the scope of the intercoder reliability check. Reliability in content analysis is achieved by defining the categories and subcategories most relevant to the study and then checking them (Riffe et al., 2005); (2) Draft the protocol. The rules and definitions that operationalize and demarcate categories and subcategories are to be outlined in a 
a coding and categorizing protocol. The protocol makes it possible for other researchers to replicate and interpret the results (Mouter \& Vonk Norrdiegraaf, 2012). (3) Determine the sample to be tested. Limiting the sample to be tested is a common procedure, as testing all the content is impractical. The sample sized is dependent upon the characteristics of the data and how large the sample should be (Mouter \& Vonk Norrdiegraaf, 2012). Lombard et al. (2004) suggest that $10 \%$ of the total content is sufficient; (4) Preform the test, choose the reliability coefficient and calculate the coefficient. The intercoder reliability check is comprised of coding and comparing the results of the coders. Reliability coefficients can be used to determine how much the data deviates from perfect reliability (Lombard et al., 2002); (5) Discuss results, choose a minimum acceptable level of reliability for the index or indices used (Mouter \& Vonk Norrdiegraaf, 2012). Lombard et al. (2002) suggest that usually scores of .90 or greater are accepted, .80 is accepted in most situations, and .70 may be accepted in some exploratory studies. 


\section{APPENDIX A}

\section{Email Verification}

(1) Table 3 and Table 4 on pages 70-71 were originally one table that was created by Tart (1975) who responded via email on August 30, 2018 and granted the use of the table.

"Charles T. Tart" < cttart@ucdavis.edu>

Re: Permission

To: Robert Anderson <randerson@ustpaul.ca>

Resent-From: randerson@ustpaul.ca

Got it! I hadn't thought of it as a table, but you're right. Yes, with credit you may certainly reproduce it.

With best wishes,

Charles Tart

(2) Figure 2 on pg. 31 created by Chalverat (2003) accepted via email on August 29, 2018 the reprinting of the Figure.

Bonjour,

L'EESP m'a fait parvenir votre demande.

Vous pouvez, bien sûr, utiliser les références de cet article.

Une version plus complète a été publiée en 1999 dans les Cahiers de psychologie analytique, La Vouivre Vol 9/ Psyché et Société aux éd. Georg, Chêne-Bourg, Genève, sous le titre:"Le mythe du guérisseur blessé dans les médecines populaires comme fondement archétypique de la relation d'aide. »

Je joins à ce message une version abrégée que je diffuse aux étudiants en médecine de l'Unil de Lausanne dans le cours que je donne sur les médecines complémentaires.

Je vous souhaite bonne réception. 
Charles Chalverat

Formateur, superviseur, consultant

Professeur Honoraire de l'EESP/HES-SO Santé/Social

Rue des Vernes 11 b

2013 Colombier

Portable : +41(0)78 7136512

(3) Figure 3 on pg. 73 was granted permission by the American Anthropological Association via email on August 10, 2018.

Hi Rob,

Thank you for this request! We would be happy to grant you permission to use the figure, free of charge, in your doctoral thesis. We do ask that you include a credit line for the figure; the one we usually provide is as follows: "Reproduced by permission of the American Anthropological Association from Anthropology of Consciousness, Volume 3, Issue 3-4, pp. 3-13, 1992. Not for sale or further reproduction." If you publish your work in the future and wish to include the figure, you will need to contact AAA again regarding permissions.

Please let me know if you have any questions! And congratulations on your upcoming thesis completion!

Sincerely,

Chelsea

Chelsea Horton

Publishing Assistant

American Anthropological Association

chorton@americananthro.org 
(4) Figure 4 was created by Krippner (1999) who granted the use of the table on August 26, 2018 via email.

Stanley Krippner <skrippner@saybrook.edu>

Re: permission to use model

To: Robert Anderson <randerson@ustpaul.ca> Cc: Steve Hart < shart@saybrook.edu>

Resent-From: randerson@ustpaul.ca

I think I already gave my permission.

Good luck

Stanley Krippner

(5) Figure 5 create by Creswell (2013) was granted permission by Sage Publishing through

Copyright Clearance Centre on September 26, 2018 via email.

\section{SAGE College LICENSE} TERMS AND CONDITIONS

Sep 28, 2018

This is a License Agreement between Rob Anderson ("You") and SAGE College ("SAGE College") provided by Copyright Clearance Center ("CCC"). The license consists of your order details, the terms and conditions provided by SAGE College, and the payment terms and conditions.

All payments must be made in full to CCC. For payment instructions, please see information listed at the bottom of this form.

License Number $\quad 4436590702441$

License date $\quad$ Sep 26, 2018

Licensed content publisher SAGE College

Licensed content title Qualitative inquiry and research design : choosing among five approaches

Licensed content date Jan 1, 2013

Type of Use Thesis/Dissertation

Requestor type Author of requested content 


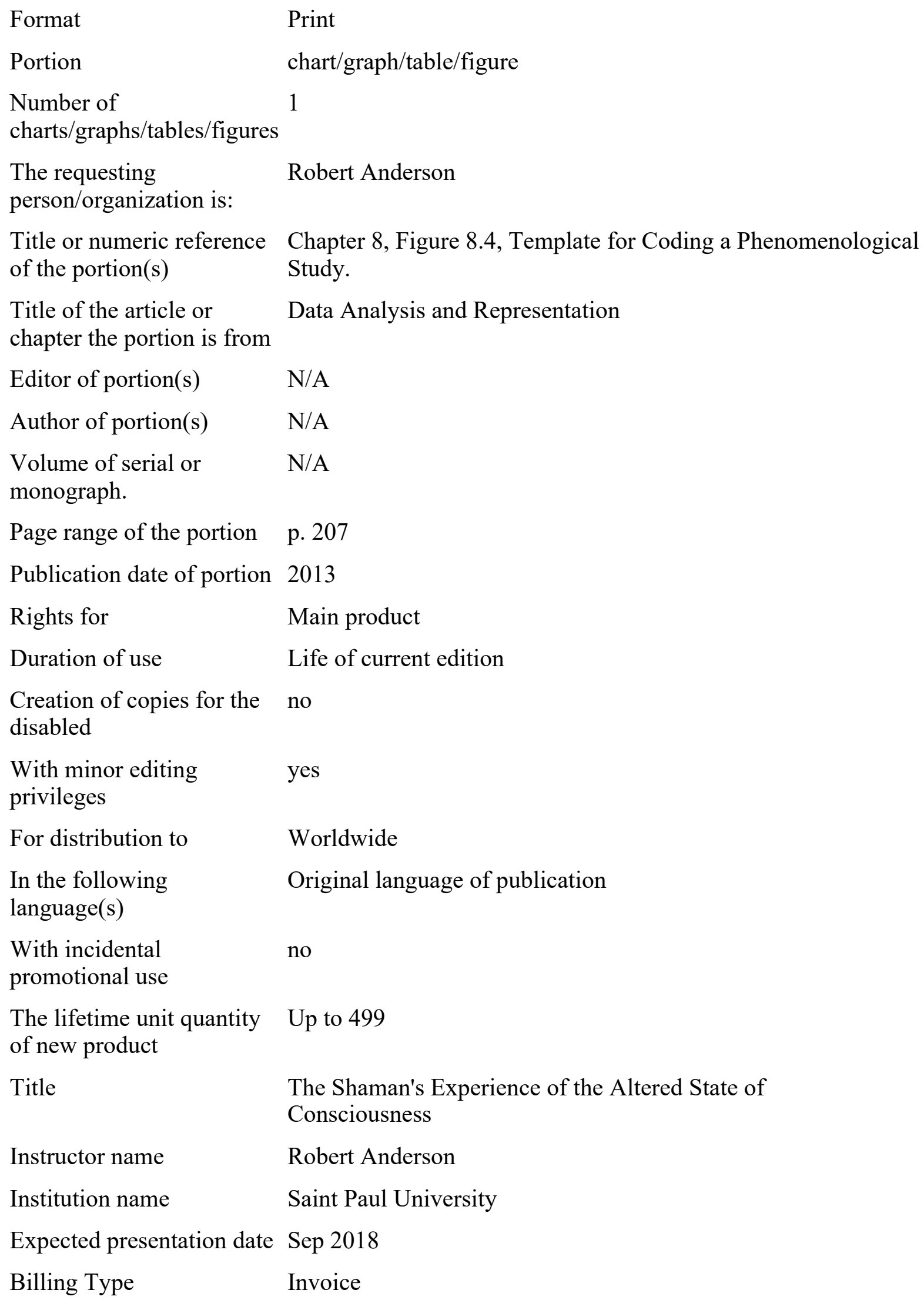




\section{Billing Address Rob Anderson \\ 28 Manhattan Crescent}

Ottawa, ON K2C4B4

Canada

Attn: Rob Anderson

Total (may include CCC $\quad 0.00$ USD user fee) 


\section{APPENDIX B}

\section{$\mathbf{S}$ \\ $\mathrm{P}$ \\ UNIVERSITÉ \\ SAINT.PAUL \\ UNIVERSITY \\ Recruitement Email}

My name is Rob Anderson. I am a PhD candidate under the supervision of Dr. Judith Malette with the School of Counselling, Psychotherapy and Spirituality at Saint-Paul University. My research study is investigating the set of described experiences that the shaman experiences while induced into the Altered State of Consciousess. I am looking for your participation in this study with the intention of adding valuable information and insight into the nature of this experience.

Your very valuable participation in this study will allow you to contribute to this knowledge base while at the same time experiencing first hand what it is like to participate in research. If it is possible like to have an initial visit so I get a sense of where you work and the setting that you use. Your confidential participation in this study will require 4 hours of your time. This can happen at your convenience over the following months between July 2015 and December 2015.

If you are interested in participating or would like ot know more about this investigation, please contact the lead researcher; Rob Anderson@ rande048@uottawa.ca; Dr. Judith Malette@ jmalette@ustpaul.ca.

Thank you for your participation and interest in this study!

Yours truly,

Rob Anderson

$\mathrm{PhD}$ Candidate

Thesis Director

Dr. Judith Malette

Saint Paul University

223 Main St. Ottawa, ON 


\section{APPENDIX C}

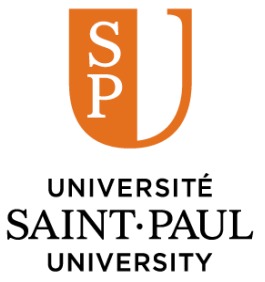

\section{Consent Information for Participants}

\section{Title of research project:}

What are the set of described experiences that the shaman experiences while induced into the Altered State of Consciousness?

\section{Description of the research project:}

This present research project aims to gain greater understanding of the shaman's experience of the altered state of consciousness. More precisely, I aim to establish an understanding of the essence behind the experience of the altered state of consciousness. Often experienced as an interaction with other phenomena that includes visual and auditory experiences, the goal of this study will help conceptualize the shaman's experience of this unique occurrence.

\section{Participation in the research project:}

If you accept to participate in this research project, your participation will consist of completing a socio-demographic questionnaire and a 3-part semi structured interview of your experience while immersed into the ASC. The socio-demographic interview should take between 30 and 40 minutes. You will be asked to email the questionnaire in an encrypted file format using the password shaman to ensure that the principle researcher and yourself will only have access. Following this the researcher will email you to arrange a time to be interviewed. The 3-part interview process should take 60 minutes each of your time. Your participation will also provide us the consent to use the results of the questions for the methodological analyses of the study.

\section{Confidentiality}

We assure you that all your answers will be strictly confidential. Anonymity and confidentiality will be assured throughout the study. Only members of the research team ( $\mathrm{PhD}$ Candidate Rob Anderson, professor: Dr. Judith Malette) will know who participated in the research and will have access to the results. Your questionnaires, once completed, will be coded even before the analysis is done and it will not be possible to identify the participant(s) by name. Data will only be analyzed to inform this research project and will have no bearing on your performance evaluations now or in the future. The data will be retained for 7 years. 


\title{
Possible advantages and disadvantages of this research:
}

Your participation in this research will not be paid. However, if a summary of the results of the study becomes available, it will be emailed to you if that is your wish. Your participation in this study will allow a greater understanding of the experience of the altered state of consciousness. If participation in this study generates in you a psychological discomfort, which is unlikely, and you would like to further explore this discomfort, we recommend that you contact your local health care authority.

\section{Your participation in this research:}

Your participation in this research is completely voluntary and you are free to withdraw from the research at any time or refuse to answer certain questions with no penalty. The results will be reported in a comprehensive manner and no names will appear in papers published from this study.

\section{Your questions:}

If you have any questions, concerns or comments about this study, please contact the principal investigator, Rob Anderson, at the following address: rande048@ottawa.ca. For information about your rights as a participant, you can contact Dr. Louis Perron, Director of the Ethics Committee for Research at Saint Paul University, to the following email address: lperron@ustpaul.ca

Thank you for your attention and please accept our warmest regards,

\author{
Rob Anderson \\ PhD Candidate \\ Saint-Paul University \\ Faculty of Human Sciences and Philosophy \\ Phone: (613) 327-1296 \\ Thesis Supervisor: \\ Judith Malette, Ph. D., C. Psych. \\ Full Professor \\ Faculty of Human Sciences and Philosophy \\ Saint Paul University \\ 223 Main St. \\ Ottawa, ON
}




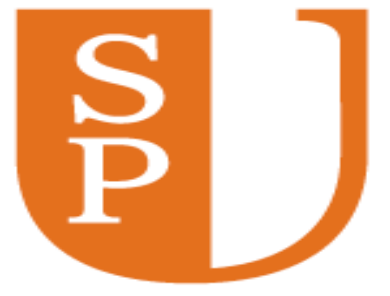

\section{UNIVERSITÉ SAINT.PAUL UNIVERSITY}

\section{Socio-demographic questionnaire}

1. Sex : Male Female

2. Age : years

3. Primary Language : English French Other (specify) :

4. Language used (language used most frequently):) : (specify) :

English French

5. In what area have you lived most of your life?

City or Town:

Province : Country :

Marital Status: Are you a ...?

Single Person

Married

Divorced/Separated

Common Law

Widowed

Other; Specify :

6. Education Level :

Secondary

College Diploma

University Diploma: under graduate

University Diploma: MA graduate degree

University Diploma: PhD graduated degree

Other, specify:

7.What is your religious denomination?

Catholic; Protestant; Jewish; Islam;

Other; Specify: 
8. Do you practice your religion? (Eg, attend Mass or other religious rites.) :

Very frequently (every day)

At least once a month

Frequently (once / week.)

At least once a month

Few times a year

Once a year

\section{Do you define yourself as a spiritual person?}

Yes

Specify how in the area you most identify with:

On the religious (tradition called and identified)

With the Universe (universal acceptance, understanding)

With myself (I am the sum of all my life experiences)

Socially (I value Humanity)

None

Thank you for your participation! 


\section{APPENDIX E}

3 stage Semi-Structured

First stage: Focused Life History

According to Seidman (2013) this

early experiences that the

phenomenon.

\section{$\underset{\mathrm{P}}{\mathrm{S}}$}

UNIVERSITÉ

SAINT.PAUL

UNIVERSITY

\section{Interview}

stage involves reconstructing the

participants have of the

\section{Questions}

1) What set of experiences led you to becoming a shaman?

a) How were these experiences important for you to understand?

b) What did you learn from these experiences?

c) How did these experiences "tell you" that you were on the path of becoming a shaman?

\section{Second Stage: The Details of Experience}

According to Seidman (2013) the second stage asks the participants to reconstruct the details of the experience of the phenomenon.

\section{Questions}

1) As a shaman, what do you experience?

a) How would you describe the experience of the ASC? For example: When you communicate with spirits; when you receive information that is about the person or group of people you are helping.

2) How has the ASC helped you understand yourself in the following ways?

a) How would you describe your experience on a physical level?

b) How would you describe your experience on an emotional level? 
c) How would you describe your experience on a spiritual level?

d) How would you describe your experience on a cognitive level?

\section{Third Stage: Reflection on Meaning}

According to Seidman (2013) the third stage asks the participants to look at how the situations in their lives interacted to bring them to their present understanding of the phenomenon.

\section{Questions}

1) Given what you have said about your life before you became a shaman and given what you have said about what you do now, how do you understand the experiences of the ASC?

a) How have these experiences led you to understand the details of the ASC?

2) Can you describe the details of this experience in the following ways?

a) Can you describe the details of this experience on a physical level?

b) Can you describe the details of this experience on an emotional level?

c) Can you describe the details of this experience on a spiritual level?

d) Can you describe the details of this experience on a cognitive level? 


\section{APPENDIX F}

\section{Certificat de déontologie Ethics Certificate Comité de la déontologie Research Ethics Board}

REB File Number

$1360.2 / 15$

Principal Investigator / Thesis supervisor / Co-investigators / Student

Last name

Anderson

Malette

Type of project

Title

Approval date
15-06-2015
(dd-mm-yyyy)

\section{Name}

Robert

Judith

\section{PhD Thesis}

The Shamman's Experience of the Altered State of Consciousness.

\section{Affiliation}

Faculty of Human Sciences

Faculty of Human Sciences
Role

PhD Student-PI

Thesis supervisor

\section{Committee comments}

The Research Ethics Board (REB) approved the project.

The researcher is invited to use the reference number 1360.2/15 when recruiting participants.

\section{General guidelines}

\begin{tabular}{ll} 
Expiry Date & Decision \\
\hline $\begin{array}{l}\text { 14-06-2016 } \\
(d d-m m-y y y y)\end{array}$ & 1 (approved)
\end{tabular}

In accordance with the Tri-Council Policy Statement, the Saint Paul University Research Ethics Board has examined and approved the application for an ethics certificate for this project for the period indicated and subject to the conditions listed above.

The research protocol may not be modified without prior written approval from the REB. This includes, among others, the extension of the research, additional recruitment for the inclusion of new participants, changes in location of the fieldwork, any stage where a research permit is required, such as work in schools. Minor administrative changes are allowed.

The REB must be notified of all changes or unanticipated circumstances that have a serious impact on the conduct of the research, that relate to the risk to participants and their safety.

Modifications to the project, information, consent and recruitment documentation must be submitted to the Office of Research and Ethics for approval by the REB.

The investigator must submit a report four weeks prior to the expiry date of the certificate stated above requesting an extension or that the file be closed.

Documents relating to publicity, recruitment and consent of participants should bear the file number of the certificate. They must also indicate the coordinates of the investigator should participants have questions related to the research project. In which case, the documents will refer to the Chair of the REB and provide the coordinates of the Office of Research and Ethics.

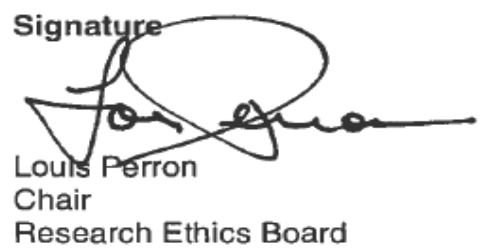


